\title{
A Comprehensive Survey of "Metamaterial Transmission-Line Based Antennas: Design, Challenges, and Applications"
}

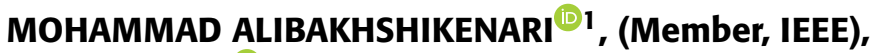 \\ BAL S. VIRDEE ${ }^{\circledR 2}$, (Senior Member, IEEE), LEYRE AZPILICUETA ${ }^{\circledR 3}$, (Senior Member, IEEE), \\ MOHAMMAD NASER-MOGHADASI ${ }^{\circledR}$, (Senior Member, IEEE), \\ MOBAYODE OLUSOLA AKINSOLU ${ }^{5}$, (Member, IEEE), \\ CHAN HWANG SEE ${ }^{\circledR 6,7}$, (Senior Member, IEEE), BO LIU ${ }^{8}$, (Senior Member, IEEE), \\ RAED A. ABD-ALHAMEED ${ }^{\circledR}$, (Senior Member, IEEE), \\ FRANCISCO FALCONE ${ }^{\circledR 10}$, (Senior Member, IEEE), \\ ISABELLE HUYNEN ${ }^{11}$, (Senior Member, IEEE), \\ TAYEB A. DENIDNI ${ }^{12}$, (Fellow, IEEE), AND ERNESTO LIMITI $^{\circledR 1}$, (Senior Member, IEEE)

This work was supported in part by the Ministerio de Ciencia, Innovación y Universidades, Gobierno de España (MCIU/AEI/FEDER, UE) under Grant RTI2018-095499-B-C31, in part by the Innovation Programme under Grant H2020-MSCA-ITN-2016 SECRET-722424, and in part by the financial support from the U.K. Engineering and Physical Sciences Research Council (EPSRC) under Grant EP/E022936/1.

\begin{abstract}
In this review paper, a comprehensive study on the concept, theory, and applications of composite right/left-handed transmission lines (CRLH-TLs) by considering their use in antenna system designs have been provided. It is shown that CRLH-TLs with negative permittivity $(\varepsilon<0)$ and negative permeability $(\mu<0)$ have unique properties that do not occur naturally. Therefore, they are referred to as artificial structures called "metamaterials". These artificial structures include series left-handed $(L H)$ capacitances $\left(C_{L}\right)$, shunt $L H$ inductances $\left(L_{L}\right)$, series right-handed $(R H)$ inductances $\left(L_{R}\right)$, and shunt $R H$ capacitances $\left(C_{R}\right)$ that are realized by slots or interdigital capacitors, stubs or via-holes, unwanted current flowing on the surface, and gap distance between the surface and ground-plane, respectively. In the most cases, it is also shown that structures based on CRLH metamaterial-TLs are superior than their conventional alternatives, since they have smaller dimensions, lower-profile, wider bandwidth, better radiation patterns, higher gain and efficiency, which make them easier and more cost-effective to manufacture and mass produce. Hence, a broad range of metamaterial-based design possibilities are introduced to highlight the improvement of the performance parameters that are rare and not often discussed in available literature. Therefore, this survey provides a wide overview of key early-stage concepts of metematerial-based designs as a thorough reference for specialist antennas and microwave circuits designers. To analyze the critical features of metamaterial theory and concept, several examples are used. Comparisons on the basis of physical size, bandwidth, materials, gain, efficiency, and radiation patterns are made for all the examples that are based on CRLH metamaterial$T L s$. As revealed in all the metematerial design examples, foot-print area decrement is an important issue of study that have a strong impact for the enlargement of the next generation wireless communication systems.
\end{abstract}

The associate editor coordinating the review of this manuscript and approving it for publication was Shah Nawaz Burokur ${ }^{\text {(D) }}$. 
INDEX TERMS Metamaterials (MTMs), artificial structures, antennas, negative permittivity $(\varepsilon<0)$, negative permeability $(\mu<0)$, high performances, composite right/left-handed transmission lines (CRLH-TLs), next generation wireless communication systems.

\section{INTRODUCTION}

Metamaterials can be best described as structures that are artificially engineered to yield certain electromagnetic properties that do not exist naturally [1]. Metamaterials characterized with both negative permittivity $(\varepsilon<0)$ and negative permeability $(\mu<0)$ are referred to as left-handed metamaterials (LHMs). LHMs have been considerably studied by scientific, technological and engineering communities [2]. In 2003, LHMs were identified by Science magazine to be in the top ten of contemporary scientific breakthroughs [3]. New applications, concepts, and devices have been developed by employing the unique characteristics of LHMs [4]. In this study, the basic electromagnetic (EM) specifications and physical implementations of LHMs are evaluated based on a transmission line (TL) method. The TL method is an efficient design approach for applications of LHMs and it provides a good insight into the physical phenomena of LHMs. In general, LHMs are described as generic models of composite right/left hand (CRLH) models that exhibit right-handed ( $\mathrm{RH})$ properties that are innate in practical LHMs. The characterization, design, and realization of $1 \mathrm{D}$ and 2D CRLH TLs are reviewed and studied. Additionally, new antenna devices based on CRLH TLs are investigated and discussed.

TL metamaterials are 1-D metamaterials comprising of the host TL loaded with reactive elements (e.g., capacitances and inductances) and/or resonators. TL metamaterials or metamaterial TLs can also be considered as a type of artificial TL [5]. Arguably, the most relevant characteristic of metamaterial TLs compared with conventional TLs is the enablement of further controllability with respect to the main line parameters (i.e., the phase constant or phase shift of the transmission line) and the characteristic impedance. As it is revealed in subsequent sections of this study, this further controllability provided by metamaterial TLs on the line parameters paves the way for the design of antenna structures with more compact dimensions, higher performances, and/or novel functionalities. As a matter of fact, metamaterial TLs and effective media metamaterials both offer added advantages over their conventional counterparts due to the controllability of their specifications beyond those accessible when their conventional alternatives are used. However, it is good to note that, in some cases creating a CRLH metamaterial TL involves some short of artificially-engineered prototyping to create the "non-natural" / artificial left-handed components: $C_{L}$ and $\mathrm{L}_{\mathrm{L}}$. This in the end complicates the structure when compared to natural right-handed, conventional, non-artificial TLs. For instance, a microstrip-line leaky-wave antenna (LWA) operating in its radiative mode (leaky mode) with conventional right-handed operation is simpler than its CRLH microstrip counterpart. Therefore, in some applications there is no such need to obtain CRLH operation [6]-[10].
Figs.1 (a), (b), and (c) show the circuit models for PRH (purely RH), PLH (purely LH), and CRLH lossless TLs, respectively. The PRH TL equivalent circuit model, shown in Fig. 1(a), is typically the combination of a series inductor $L_{R}$ and a shunt capacitor $C_{R}$. The PLH TL circuit model, exhibited in Fig. 1(b), is the dual counterpart of the PRH TL. The PRH TL is a synergy of a times-unit length shunt inductor, $L_{L}$ and a times-unit length series capacitance, $C_{L}$. For practical purposes, the realization of a PLH structure is not feasible. This is due to the unavoidable $\mathrm{RH}$ parasitic series inductor $\left(L_{R}\right)$ and shunt capacitor $\left(C_{R}\right)$ effects (parasitic capacitance resulting from voltage gradients, and inescapable parasitic inductance resulting from the flow of current in the direction of metallization). Thus, a CRLH-structure introduces a very generic configuration of a structure with $\mathrm{LH}$ properties. The generic CRLH TL equivalent circuit model exhibited in Fig.1(c) comprises of a series combination of an inductance $L_{R}$ and a capacitance $C_{L}$, and a shunt capacitor $C_{R}$ in parallel with an inductor $L_{L}$.

The propagation constant $(\gamma)$ of a transmission line (TL) is evaluated as: $\gamma=\alpha+j \beta=\sqrt{Z Y}$, where $Y$ and $Z$ represent the admittance and impedance, respectively. Considering the CRLH-TL, $Z$ and $Y$ can be evaluated as follows [1], [2], [11]:

$$
\begin{aligned}
& Z(\omega)=j\left(\omega L_{R}-\frac{1}{\omega C_{L}}\right) \\
& Y(\omega)=j\left(\omega C_{R}-\frac{1}{\omega L_{L}}\right)
\end{aligned}
$$

So, a homogenous CRLH TL will have the following dispersion relation:

$$
\beta(\omega)=s(\omega) \sqrt{\omega^{2} L_{R} C_{R}+\frac{1}{\omega^{2} L_{L} C_{L}}-\left(\frac{L_{R}}{L_{L}}+\frac{C_{R}}{C_{L}}\right)}
$$

where

$$
s(\omega)\left\{\begin{array}{l}
-1 \quad \text { if } \omega<\omega_{\Gamma 1}=\min \left(\frac{1}{\sqrt{L_{R} C_{L}}}, \frac{1}{\sqrt{L_{L} C_{R}}}\right) \\
+1 \quad \text { if } \omega>\omega_{\Gamma 2}=\max \left(\frac{1}{\sqrt{L_{R} C_{L}}}, \frac{1}{\sqrt{L_{L} C_{R}}}\right)
\end{array}\right.
$$

In Eqn.(3), the phase constant, $\beta$ can either be purely real or purely imaginary depending on the sign convention the radicand assumes (i.e., plus or minus, respectively). If $\beta$ is solely real over a frequency range, a pass-band is present in that frequency range due to the condition, $\gamma=j \beta$. On the other hand, if $\beta$ is purely imaginary over a frequency range, a stop-band is present in that frequency range due to the condition, $\gamma=\alpha$. Such a stop-band is a unique to the CRLH-TL and does not exist in the PRH-TL or the PLH-TL. Although, in conventional PRH-periodic TL stop-band occurs due to space-harmonic Floquet-mode coupling, and PRH-periodic TL can balance the dispersion to obtain seamless backward-to-forward scanning. 


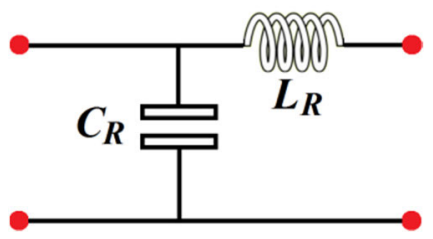

(a)

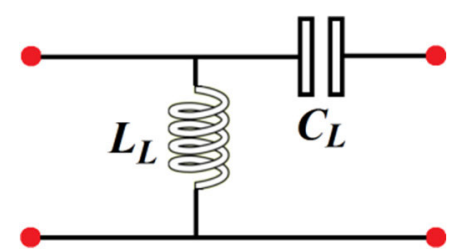

(b)

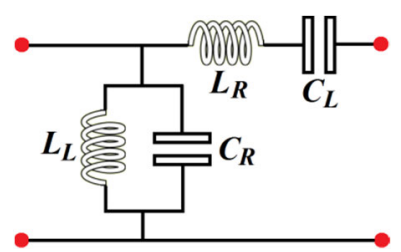

(c)

FIGURE 1. Circuit model of the (a) RH transmission line, (b) LH transmission line, and (c) CRLH transmission line.

This balancing condition is somehow similar to the condition to suppress the open-stop band (OSB) occurring in conventional periodic LWAs [12], [13]. Figs. 2 (a), (b), and (c) show the dispersion curve (i.e., $\omega-\beta$ relationship) of a PRH-TL, PLH-TL, and CRLH-TL, respectively. From the dispersion curves, the deduction and evaluation of the group velocity $\left(v_{g}=\partial \omega / \partial \beta\right)$ and the phase velocity $\left(v_{p}=\omega / \beta\right)$ of these TLs can be made. For a PRH-TL, it can also be inferred from the curves that $v_{g}$ and $v_{p}$ are parallel (i.e., $v_{p} v_{g}>0$ ). Whereas for a PLH-TL, the curves indicate that $v_{g}$ and $v_{p}$ are not parallel (i.e., $v_{p} v_{g}<0$ ). The CRLH-TL's dispersion curve also shows the presence of LH (i.e., $v_{p} v_{g}<0$ ) and RH (i.e., $v_{p} v_{g}>0$ ) regions. From Fig. 2(c), it can be seen that for a CRLH-TL, if $\gamma$ is purely real, a stop-band is present.

Generally, the series and shunt resonances of the CRLH-TL are distinct. This is referred to as the unbalanced case. However, the LH and RH contribution balance out at a given frequency when the series and shunt resonances are the same.

$$
L_{R} C_{L}=L_{L} C_{R}
$$

Hence, a balanced case condition is obtained as a result, and the resulting simplified equivalent circuit model is displayed in Fig. 3(a). For the condition in Eqn.(5), the propagation constant in Eqn.(3) decreases to a simpler expression from which Eqn.(6) is derived.

$$
\beta=\beta_{R}+\beta_{L}=\omega \sqrt{L_{R} C_{R}}-\frac{1}{\omega \sqrt{L_{L} C_{L}}}
$$

where the phase constant, $\beta$ is the combination of the RH-phase constant $\left(\beta_{R}\right)$ and the LH-phase constant $\left(\beta_{L}\right)$ when it is distinctly split.

As the frequency increases, the CRLH-TL becomes dispersive increasingly. This is because the phase velocity (i.e., $v_{p}=\omega / \beta$ ) now possesses an increasing dependence on the frequency, as expressed in Eqn.(6). On the contrary, other types of periodic-LWAs can control their dispersion to tune the frequency-scanning ratio (angle of radiation versus frequency slope), using some engineering methods [14], [15]. Apart from frequency-scanning, it is interesting to perform fixed-frequency electronic scanning with LWAs. Apart from the electronically-scannable CRLH LWAs, conventional (non CRLH) electronically reconfigurable LWA which also perform backward-to-forward electronic scanning using tunable $\mathrm{RF}$ components, and without the need of complex CRLH balanced unit-cell design [16], [17]. Therefore, in the case of CRLH it is challenging to control the scanning ratio while keeping the balancing condition. Nevertheless, in [18] and [19] the proposed CRLH LWAs have controlled the frequency-scanning sensitivity / speed / ratio applying some interesting approaches. Eqn.(6) shows that at low frequencies, the CRLH-TL is LH-dominant, while at high frequencies, the CRLH-TL is RH-dominant. This depicts the dual nature of the CRLH- transmission line. The dispersion diagram for the balanced CRLH- transmission line is exhibited in Fig. 3(b). From Fig. 3(b), the transition from LH to RH happens at:

$$
\begin{aligned}
\omega_{0} \stackrel{\text { Unbalanced }}{\longrightarrow} \frac{1}{\sqrt[4]{L_{L} C_{L} L_{R} C_{R}}} \stackrel{\text { Balanced }}{\longrightarrow} \frac{1}{\sqrt{L C}} \\
L=L_{L}+L_{R} \\
C=C_{L}+C_{R}
\end{aligned}
$$

where $\omega_{0}$ is the transition frequency. Therefore, for the balanced case, there is a smooth transition takes place from LH to RH, since $\gamma$ is purely imaginary, in contrast to the unbalanced case. Consequently, a stop-band is not present for the balanced CRLH-TL's dispersion. Although $\beta$ is null at $\omega_{0}$ (relative to an infinite guided wavelength (i.e., $\lambda_{g}=2 \pi /|\beta|$ ), due to a non-zero $v_{g}$ at $\omega_{0}$, wave propagation still occurs. Also, at $\omega_{0}$, the phase shift is null for a TL of length $d$ (i.e., $\varphi=-\beta d=0$ ). In the LH frequency range (i.e., $\omega<\omega_{0}$ ), phase lead (i.e., $\varphi>0$ ) occurs and in the RH frequency range (i.e., $\omega>\omega_{0}$ ), phase delay (i.e., $\varphi<0$ ) occurs.

The characteristic impedance of a transmission line is presented by $Z_{0}=\sqrt{Z Y}$. For the CRLH TL, the characteristic impedance is stated as follows [1], [2], [11]:

$$
\begin{aligned}
& Z_{0} \stackrel{\text { Unbalanced }}{\longrightarrow} Z_{L} \sqrt{\frac{C_{L} L_{R} \omega^{2}}{L_{L} C_{R} \omega^{2}}} \stackrel{\text { Balanced }}{\longrightarrow} Z_{L}=Z_{R} \\
& Z_{L}=\sqrt{\frac{L_{L}}{C_{L}}} \\
& Z_{R}=\sqrt{\frac{L_{R}}{C_{R}}}
\end{aligned}
$$

where $Z_{L}$ and $Z_{R}$ are the PLH and PRH impedances, respectively.

For the unbalanced case, the characteristic impedance is dependent on the frequency dependent. Eqn.(8) shows that the balanced case is independent of the frequency 


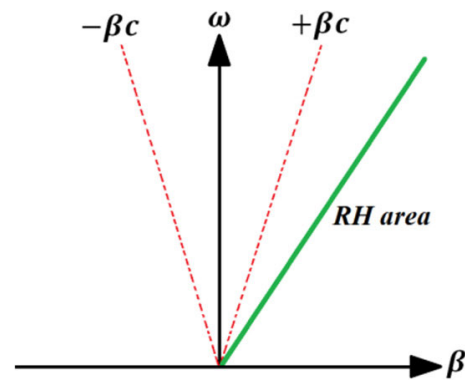

(a)

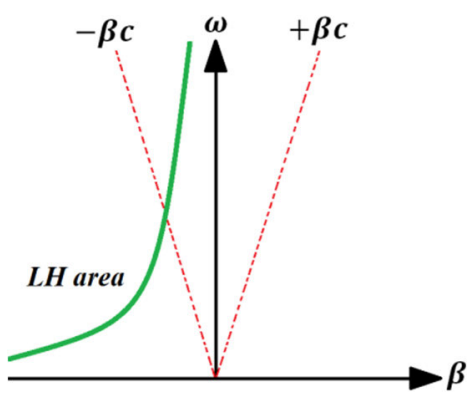

(b)

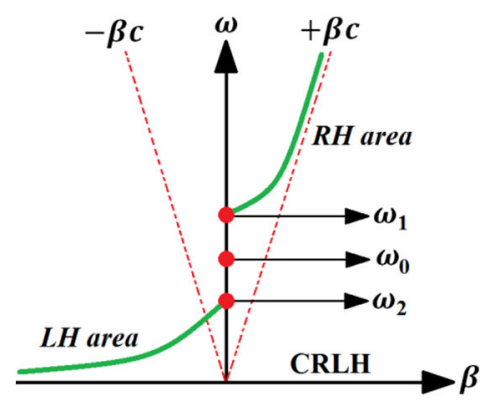

(c)

FIGURE 2. Dispersion curves for the transmission lines of the Fig.1. (a) RH transmission line, (b) LH transmission line, and (c) CRLH transmission line (unbalanced).

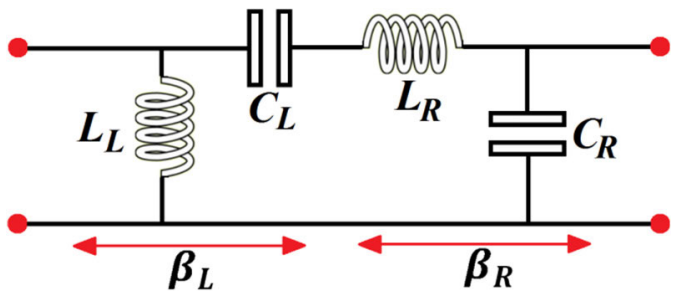

(a)

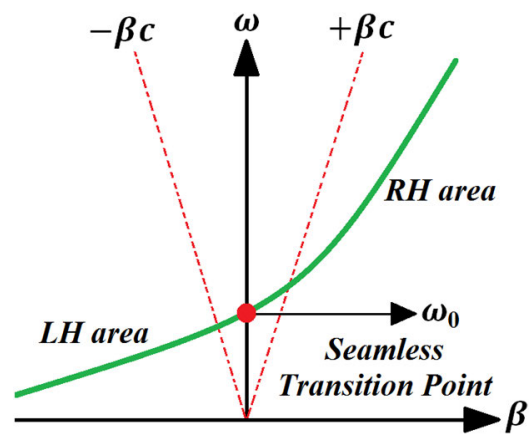

(b)

FIGURE 3. Balanced case of the Fig.1(c). (a) simplified circuit model, and (b) dispersion curve displaying seamless LH to RH transition.

independent and thus, it is possible to have a broad bandwidth matching.

The derived TL equations above are analogous to the constitutive parameters that define a CRLH material. The propagation constant of a TL as stated earlier is $\gamma=j \beta=$ $\sqrt{Z Y}$. Given the propagation constant of a material $(\beta=$ $\omega \sqrt{\varepsilon \mu}$ ), the equation in below can be derived [1], [2], [11]:

$$
-\omega^{2} \varepsilon \mu=Z Y
$$

Similarly, the characteristic impedance of the TL, i.e., $Z_{0}=$ $\sqrt{Z Y}$ is analogous to the intrinsic impedance of the material, i.e., $\eta=\sqrt{\mu / \varepsilon}$ and it is expressed as follows:

$$
Z_{0}=\eta \quad \text { or } \frac{Z}{Y}=\frac{\mu}{\varepsilon}
$$

Eqn.(11) is analogous to the permeability and permittivity of a material, and the impedance and admittance of its transmission line model.

$$
\begin{aligned}
\mu & =\frac{Z}{j \omega}=L_{R}-\frac{1}{\omega^{2} C_{L}} \\
\varepsilon & =\frac{Y}{j \omega}=C_{R}-\frac{1}{\omega^{2} L_{L}}
\end{aligned}
$$

Fig. 4 shows the refractive index (i.e., $n=c \beta / \omega$ ) for the balanced and unbalanced CRLH-TL. In Fig.4, the CRLH-TL has a negative refractive index in its LH-range and a positive refractive index of refraction in its RH-range.

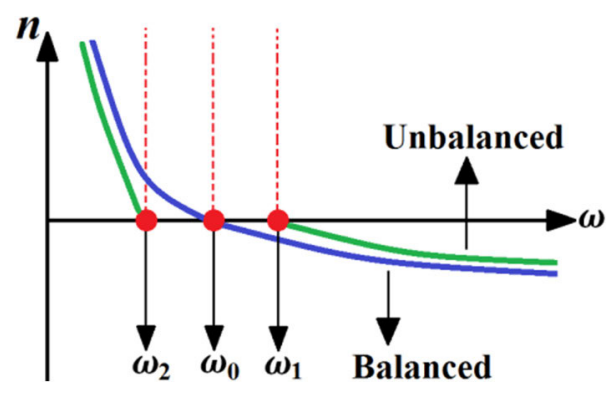

FIGURE 4. Typical index of refraction diagrams for the balanced- and unbalanced-CRLH TLs in blue and green, respectively.

\section{A. LC NETWORK}

Naturally, homogeneous CRLH-TLs do not exist. However, when an EM wave is not "impaired" by discontinuities in the propagating structure or medium since the guided wavelength is considerably larger compared, in a certain range of frequencies CRLH-TLs can be effectively made to be homogenous and can be physically implemented. A typical CRLH-TL of length $d$ that is effectively homogenous can be built through a cascade of the band-pass $L C$ unit cell of Fig.5(a), periodically or non-periodically. Typically, to ensure computational and manufacturing convenience of the CRLH-TL, periodicity is required [11]. The unit cell 


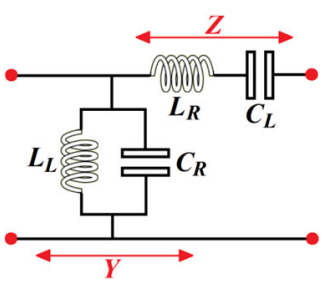

(a)

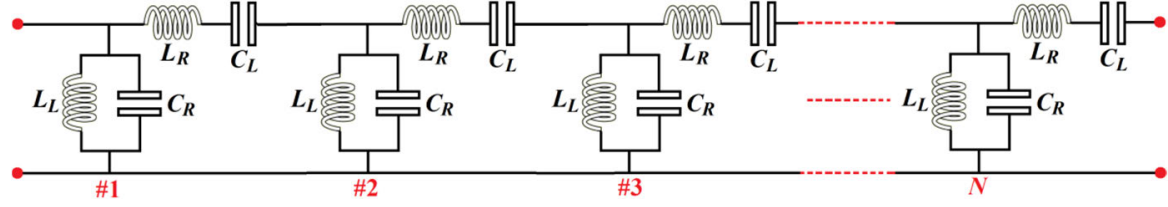

(b)

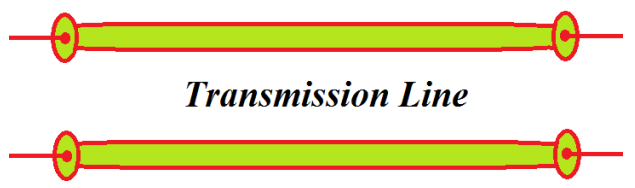

(c)

FIGURE 5. LC-based CRLH TL. (a) Unit-cell. (b) LC periodic network, and (c) homogeneous CRLH TL, which is equivalent to (b).

of Fig.5(a) has no dimensions compared to the model of Fig. 1(c) which is incremental with an infinitesimal physical length (i.e., $\Delta z$ in meters). By considering its electrical length, $\theta=\Delta \varphi(\mathrm{rad})$, the $L C$ unit cell's phase can be illustrated. However, for a practical realization of the applied inductors and capacitors, a physical length $p$ needs to be established. The choice of applied technology (e.g., microstrip, coplanar waveguide, surface mount components, etc.) impacts on the physical dimensions of the unit cell of the $L C$. The $L C$ unit-cell of Fig. 5(a) is similar to the incremental model of Fig. 1(c) for the limit $p=\Delta z \rightarrow 0$. According to the homogeneity condition $p \rightarrow 0$ in Fig.5(b), it is possible to construct a TL (by cascading the $L C$ unit-cell) equivalent to an ideal homogeneous CRLH-TL of length $d$ [11]. TL is made to appear homogeneous to the EM wave as a result of the homogeneity condition. In practice, the electrical length of the unit cell becomes less than $\pi / 2$ and the $L C$-based CRLH-TL appears to be effectively homogeneous by the EM waves if and/or when the unit cell is less than the guided wavelength (i.e., $p<\lambda_{g} / 4$ ) [11].

For the LC unit cell, considering the periodic boundary conditions (PBCs) which are similar to the Bloch-Floquet theorem [11], the dispersion relation (for the LC unit cell) is justified and expressed as follows [1], [2], [11]:

$$
\beta(\omega)=\frac{1}{p} \cos ^{-1}\left(1+\frac{Z Y}{2}\right)
$$

where the series impedance $(Z)$ and shunt admittance $(Y)$ of the $L C$ unit-cell are determined by:

$$
Z(\omega)=j\left(\omega L_{R}-\frac{1}{\omega C_{L}}\right), \quad Y(\omega)=j\left(\omega C_{R}-\frac{1}{\omega C_{L}}\right)
$$

The Taylor approximation $\cos (\beta p) \approx 1-(\beta p)^{2} / 2$ can employed due to the small electrical length of the unit cell; as a result, Eqn.(15) becomes:

$$
\beta(\omega)=\frac{s(\omega)}{p} \sqrt{\omega^{2} L_{R} C_{R}+\frac{1}{\omega^{2} L_{L} C_{L}}-\left(\frac{L_{R}}{L_{L}}+\frac{C_{R}}{C_{L}}\right)}
$$

Eqn.(17) is equivalent to Eqn.(3) which describes the homogeneous dispersion relation. Therefore, for electrical lengths, the LC-based CRLH-TL and homogeneous CRLH-TL are analogous.

\section{B. PHYSICAL IMPLEMENTATION}

In the preceding subsection, the $L C$ method to generate a CRLH-TL has been demonstrated and discussed. This $L C$ network can only be realized by employing physical components that are able to yield the required capacitors $\left(C_{R}\right.$ and $\left.C_{L}\right)$ and inductors $\left(L_{R}\right.$ and $\left.L_{L}\right)$. In recent times, the application of surface-mount technology (SMT) chip components or distributed components is attracting a lot of interest for the implementation of such an $L C$ network. Microstrip, strip line, coplanar waveguide, or other similar technologies can be used to realize distributed components.

Numerous factors inform the choice of SMT chip or distributed components. CRLH structures based on SMT components are quite commonly and simpler to realize efficiently in terms of analysis and design. This is because of the availability of ready-to-use SMT chip components which do not need to be modelled and manufactured compared to the distributed components. However, the availability of SMT components is in discrete quantities and they are generally operational only at low frequencies (i.e., 3-6 GHz, corresponding on their amounts). As a result, SMT-based CRLH-structures are characterized with limited operational frequency ranges and specific phase. It's an application-specific choice between SMT chip components and distributed components. For instance, in radiation-type applications, SMT chip components may be infeasible.

Fig.6. shows a CRLH-TL based distributed component. The structural implementation is via microstrip having interdigital capacitors and stub inductors linked to the GND. The equivalent circuit model of Fig. 5(a) and the unit-cell of the structure (exhibited in the inset) are analogous. LH and the RH effects are produced by the interdigital capacitors and stub inductors. Particularly, the LH capacitance $C_{L}$ is 


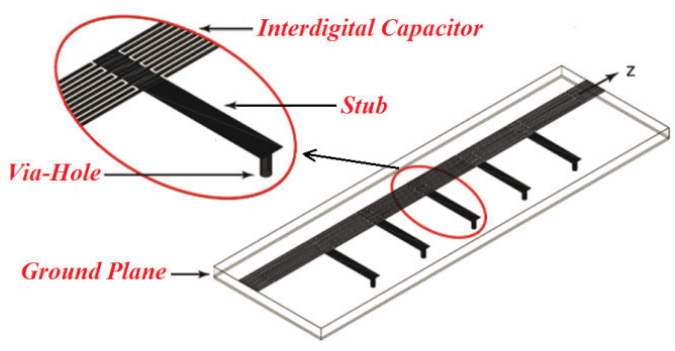

FIGURE 6. 1-D microstrip CRLH TL constructed of interdigital capacitors and shorted-stub inductors [11].

realized by the interdigital capacitors and the $\mathrm{LH}$ inductance $L_{L}$ results from the stubs linked by means of via-holes to the ground plane. The capacitance that exists between the trace and GND assumes the RH capacitance $C_{R}$, and the inductance resulting from by the magnetic flux produced by the flow of current in the digits of the interdigital capacitor assumes the RH inductance, $L_{R}$.

By loading a host line with split ring resonators (SRRs) or with other similar resonators similar, CRLH-TL can also be realized. Martin et al. proposed the first SRRs based LH-TL in 2003 [1], [20]. The line described in [1] is a coplanar waveguide (CPW) with etched (on the backside of the substrate and beneath the slots) pairs of SRRs and inductive shunt connecting strips. Although the backward nature of wave propagation adjacent to the resonance frequency of the SRRs is detailed in [21], the structure mainly portrays a CRLH behaviour due to properties of the host CPW TL. In [1], the equivalent model of these SRR based CRLH-lines (unit-cells) is provided. When complementary SRRs or CSRRs are etched in the ground plane, underneath a conductor strip with gaps in series [22]-[24], resonant-type CRLH TL based on microstrip technology can be realized the conductor strip [25].

The combination of open SRRs or OSRRs [26] and open CSRRs or OCSRRs [27] can also be used to implement resonant type CRLH TLs. Open resonators show half the resonance frequency (when considering similar size and substrate) compared to SRRs or CSRRs. As a result, their electrical size is smaller (by a factor of two) [26]-[28]. This property makes OSRRs and OCSRRs attractive for the miniaturization of devices. References [26], [27] provide typical topologies and equivalent circuit models for OSRR and OCSRR loading of CPW structures. Basically, the OSRR and the OCSRR can be explained using a resonator placed in series and parallel, respectively. However, some phase shift which ought to be accounted for to ensure an efficient design of the structures is introduced by the host line. The structural models are mainly composed of compounded resonators having parasitic reactive elements (i.e., capacitor and inductor) for the OSRR loaded CPW and the OCSRR-loaded CPW. Due to the presence of these parasitic reactive elements, it is not feasible to implement canonical CRLH-structures using a cascade of OSRR and
OCSRR-loaded CPW section. However, these parasitic reactive elements assume low values, and in practice, a good approximation of the canonical CRLH behaviour is observed for OSRR- and OCSRR-based lines. In comparison to SRR or CSRR loaded lines, the primary distinction is that there is no transmission zero in left direction of the left-handed band. The accuracy of the circuit models is also another vital consideration. The open particles (OSRRs and OCSRRs) possess a smaller electrical size. As a result, the equivalent circuit models of the structures based on OSRR and OCSRR give a more accurate analysis up to higher frequencies in comparison to the models for the lines loaded with SRR or CSRR. Reference [27] portrays a typical OSRR and OCSRR CRLH CPW TL.

\section{COMMMON AND RELEVANT APPLICATIONS OF MTM-TL TO ANTENNA SYSTEMS}

This section will focus on artificial metamaterial TLs for the realization of various cost effective, easy to design and manufacture, and mass produce antenna structures with miniaturized dimensions, wide bandwidth, high radiation gain and efficiency, wide range of scanning ability, and low profile, and some of their most common and relevant applications [29], [30]. In below they have been studied, reviewed, and discussed.

\section{A. BROADBAND AND MULTIBAND ANTENNAS}

Considering broadband antennas, for their design, the operative bandwidths are specified over the frequency range where the desired specifications are met within the required limits. The phase shift resulting from variations in the frequency from the nominal operating values restricts the bandwidth in distributed circuits experienced by TLs and stubs. In a typical TL having a length $l$, the electrical length (or phase) of the line at a given angular frequency $\omega_{0}$ can be obtained as follows:

$$
\varphi_{0}=\beta l=\frac{l}{v_{p}} \omega_{0}
$$

where $v_{p}$ connotes the line's phase velocity of the line. From the aforementioned, the bandwidth closely corresponds to the group delay which is evaluated as the derivative of $\varphi$ with respect to frequency. Consequently, it follows on that the bandwidth becomes broader as the line becomes shorter. In other words, an inverse relationship exists between the bandwidth and the essential phase of the line that is design specific. This is indicative of the fact that in traditional distributed circuits, the operational bandwidth cannot be easily controlled manipulated. This can be attributed to the limitations of conventional TLs in terms of the degrees of freedom. However, the loading elements allow for extra parameters in metamaterial TLs, and to an extent the phase response can be manipulated. Under normal conditions, it is expected that the bandwidth will not be determined by the required phase. In other words, to enhance the bandwidth, it becomes essential to have similar slopes in the proximity of the operational frequency for the dispersion characteristics. 
Artificial metamaterial TLs can be employed to achieve this. Following this method, numerous antenna devices with increased bandwidths have been proposed in [31]-[50].

In [51] two wideband antennas loaded with split ring resonators have been designed and manufactured (see Fig.7). The results exhibited in Fig.7 demonstrate that after loading the conventional monopole antennas to the asymmetrical meander-lines split ring resonators the lower resonance frequency modes have been stimulated. The split ring resonator's dimensions have been modified to achieve a resonance near the monopole antenna's resonance. It has been shown that when both resonance coincide the antenna's frequency bands and radiation characteristics have been increased. The antenna's length and width are $0.25 \lambda_{0} \times 0.11 \lambda_{0}$ and $0.25 \lambda_{0} \times 0.21 \lambda_{0}$ at $4 \mathrm{GHz}$ for monopole antennas, and $0.29 \lambda_{0} \times 0.21 \lambda_{0}$ at $2.9 \mathrm{GHz}$ for both monopole antennas loaded with split ring resonators. For conventional F- and T-shaped antennas with no split ring resonators the highest measured gain and radiation efficiency are $3.6 \mathrm{dBi}-78.5 \%$, and $3.9 \mathrm{dBi}-80.2 \%$, respectively, which have been occurred at $5 \mathrm{GHz}$. For antennas loaded with split ring resonators, these parameters are $4 \mathrm{dBi}-81.2 \%$, and $4.4 \mathrm{dBi}-\sim 83 \%$ for $\mathrm{F}$ - and $\mathrm{T}$ - antennas, respectively, which have been appeared at $6 \mathrm{GHz}$. By realizing the meander-lines split ring resonators as a matching load on the monopole antennas they can support the frequency bands from $2.9 \mathrm{GHz}$ to $6.41 \mathrm{GHz}$ and $2.6 \mathrm{GHz}$ to $6.6 \mathrm{GHz}$, which are corresponded to $75.4 \%$ and $\sim 87 \%$ fractional bandwidths, respectively. These achievements show $\sim 2.4$ and 2.11 times improvements on the measured bandwidths in comparison with the conventional monopole antennas with approximately fixed dimensions.

As shown in Fig.8, the experimental results of an electrically compact printed monopole antenna have been elaborated in [52] with the operational bandwidth of $185 \%$ $(0.115-2.90 \mathrm{GHz})$ for $S_{11} \leq-10 \mathrm{~dB}$, peak gain and radiation efficiency of $2.35 \mathrm{dBi}$ and $78.8 \%$, occurred at $1.45 \mathrm{GHz}$. The antenna's layout is approximated to a back-to-back triangular formed patch structure that is stimulated by a common feed-line with a meander-line T-form divider. The truncated GND contains a central stub placed under the feed-line. The antenna's bandwidth has enlarged with the inclusion of meander-line slits in the patch and four double split-ring resonators realized on the GND. The antenna radiates approximately omnidirectionally to provide coverage throughout the large parts of VHF and S-bands, and the entire parts of the UHF and L-bands. The antenna with physical dimensions of $48.32 \times 43.72 \times 0.8 \mathrm{~mm}^{3}$ and the electrical size of $0.235 \lambda_{0} \times 0.211 \lambda_{0} \times 0.003 \lambda_{0}$ has advantages of low-profile and low-cost, which make it potential candidate for applications in wideband wireless communications systems.

A planar antenna structure constructed of two pairs of interconnected meandered-line loops grounded to a truncated T-formed ground-plane through two via holes has been exhibited in Fig.9 [53]. The T-shaped GND has applied as a reflector to improve the antenna's performance

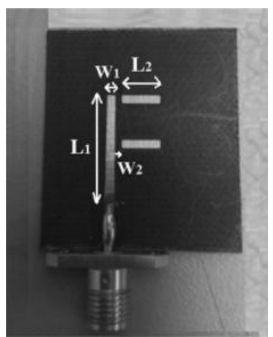

(a)

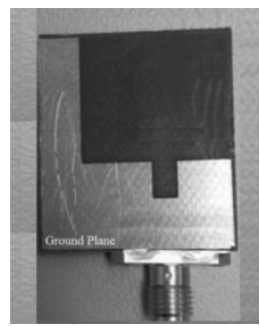

(c)

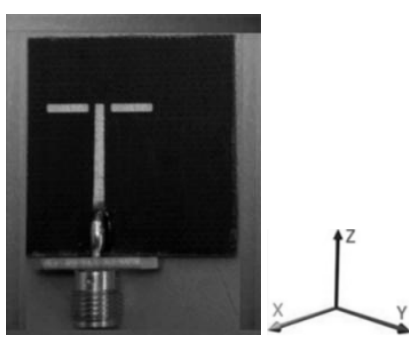

(b)

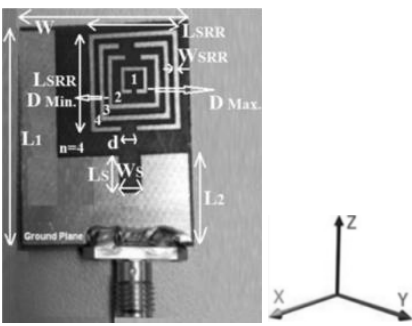

(d)

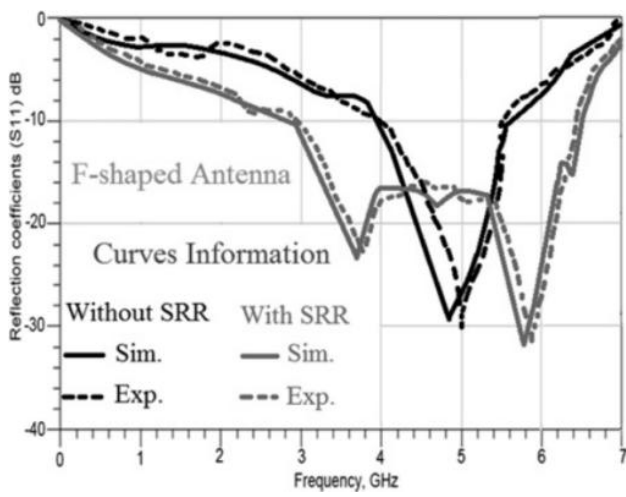

(e)

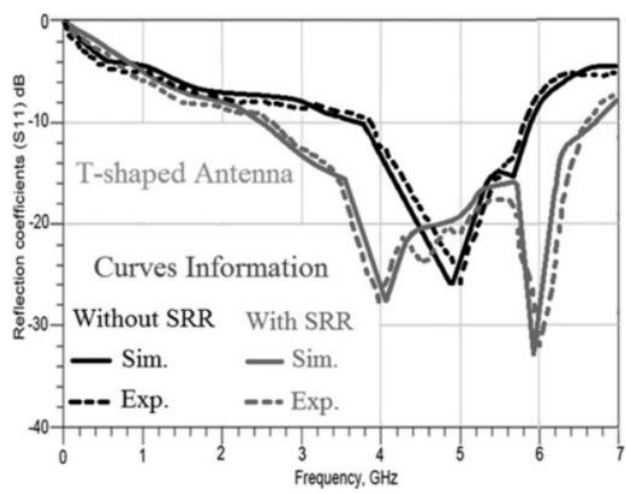

(f)

FIGURE 7. Antennas loaded split ring resonators. (a) F-antenna (top-surface), (b) T- antenna (top-surface), (c) bottom-side of both antennas with no split ring resonators, (d) bottom-side of both antennas loaded with split ring resonators, (e) $S_{11} \leq-10 \mathrm{~dB}$ for the F-antenna, (f) $S_{11} \leq-10 \mathrm{~dB}$ for the T-antenna, (g) radiation properties for the F-antenna, and (h) radiation properties for the T-antenna [51].

parameters. The antenna is miniaturized occupying an space of $38.5 \times 36.6 \mathrm{~mm}^{2}\left(0.070 \lambda_{0} \times 0.067 \lambda_{0}\right)$, where $\lambda_{0}$ is the free-space wavelength at $0.55 \mathrm{GHz}$. The antenna radiates 


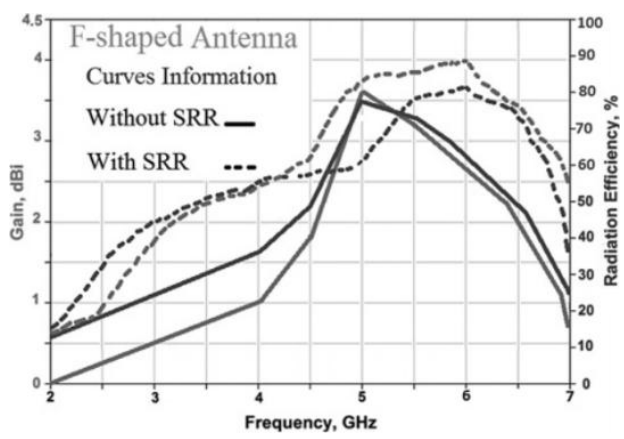

(g)

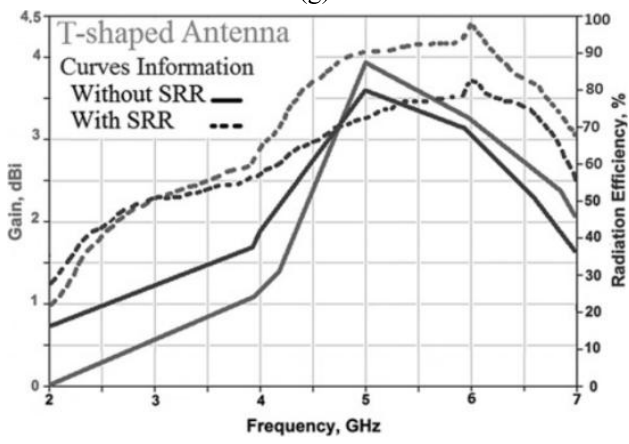

(h)

FIGURE 7. (Continued.) Antennas loaded split ring resonators. (a) F-antenna (top-surface), (b) T- antenna (top-surface), (c) bottom-side of both antennas with no split ring resonators, (d) bottom-side of both antennas loaded with split ring resonators, (e) $S_{11} \leq-10 \mathrm{~dB}$ for the F-antenna, (f) $S_{11} \leq-10 \mathrm{~dB}$ for the T-antenna, $(\mathrm{g})$ radiation properties for the F-antenna, and $(h)$ radiation properties for the T-antenna [51].

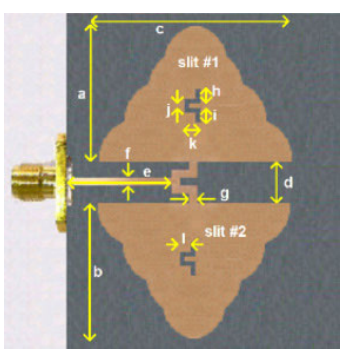

(a)

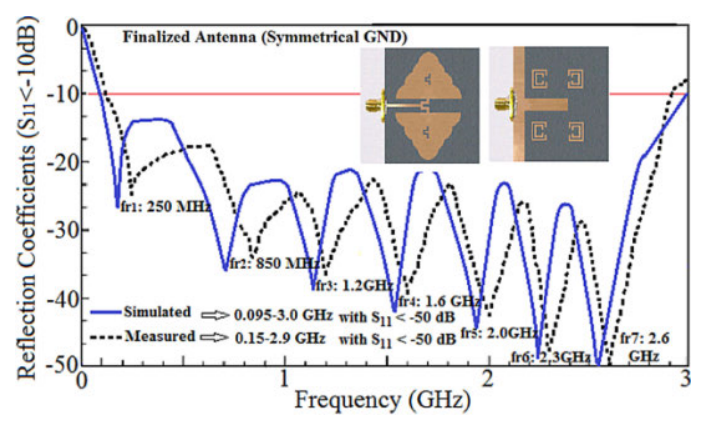

(c)

FIGURE 8. (a) Fabricated prototype and (b) simulated and measured reflection coefficient $S_{11} \leq-10 \mathrm{~dB}$ [52].

omnidirectionally in the E-plane over its working frequency band of $0.55-3.85 \mathrm{GHz}$ with a maximum gain and efficiency of $5.5 \mathrm{dBi}$ and $90.1 \%$, respectively, happened at $2.35 \mathrm{GHz}$.

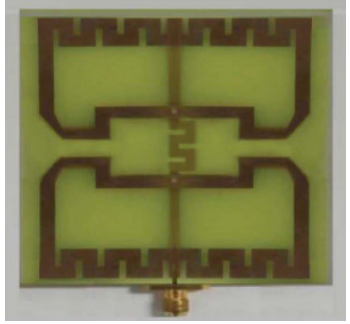

(a)

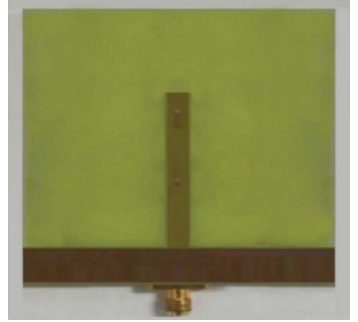

(b)

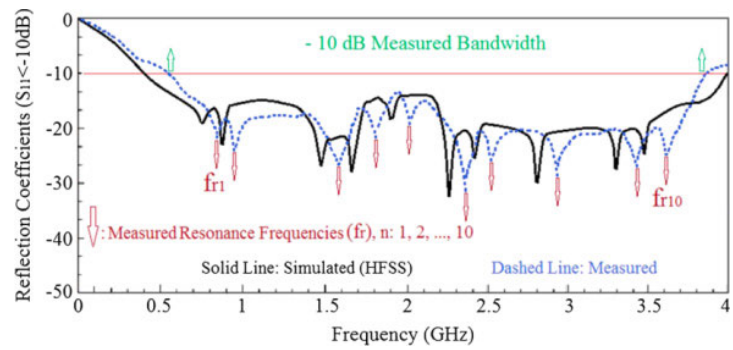

(c)

FIGURE 9. Proposed meandered antenna, (a) top surface, (b) back-side, and (c) reflection coefficients [53].

These features make the proposed antenna proper for various applications in particular JCDMA, UHF RFID, GSM 900, GPS, KPCS, DCS, IMT-2000, WiMAX, WiFi, and Bluetooth.

In [54] a approach has been applied to enlarge the frequency band of antennas without compromising the physical dimensions. This is achieved by embedding capacitive slots in the rectangular patch with a truncated GND, and stimulating the antenna via a meandered strip-line feed as depicted in Fig.10. The proposed structure has been manufactured on the FR-4 layer with $\varepsilon_{r}$ of 4.6 , thickness of $0.8 \mathrm{~mm}$, and $\operatorname{tang} \delta$ of 0.001 . Antenna covers an frequency bandwidth of $5.25 \mathrm{GHz}$ from 0.8 to $6.05 \mathrm{GHz}$, which corresponds to a practical bandwidth of $153.28 \%$ with a peak gain of $5.35 \mathrm{dBi}$, maximum radiation efficiency of $84.12 \%$, and low crosspolarization. These achievements capable the antenna to be used in stable and reliable multiband applications across the UHF, L-, S- and major part of C- bands. The antenna offers benefits of low profile, low cost, ease of manufacturing, durability and conformability.

In [55] MTM-based transmission lines have been applied to antenna systems to improve their performance characteristics. The concept of MTM and its applications to antenna structures have been described in [55]. Fig.11 presents two novel ultra-wideband (UWB) compact patch antennas modeled based on the CRLH-TLs. By applying the CRLH principle the size reduction and frequency bandwidth expansion have been obtained. additionally, a large frequency bandwidth and good radiation properties have been achieved by optimizing the dimensions of the structures. Two different types of radiators are investigated, i) a planar patch antenna compound of four O-shaped unit-cells, and ii) a planar patch antenna built of six $\mathrm{O}$-formed unit-cells. The performance 


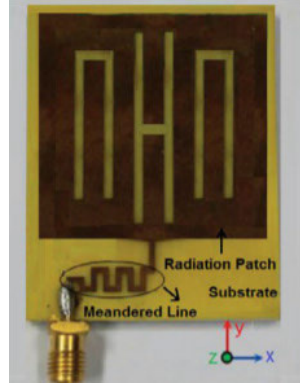

(a)

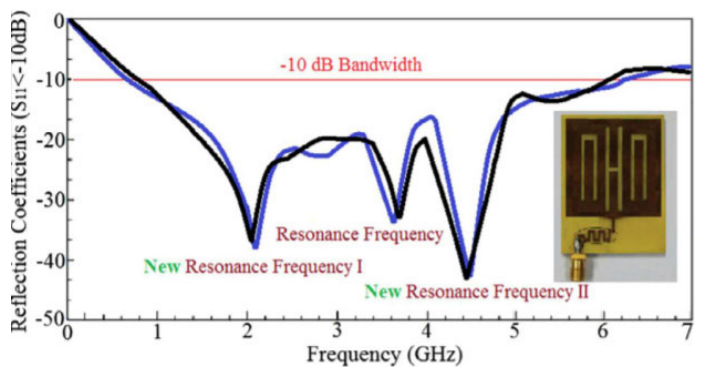

(c)

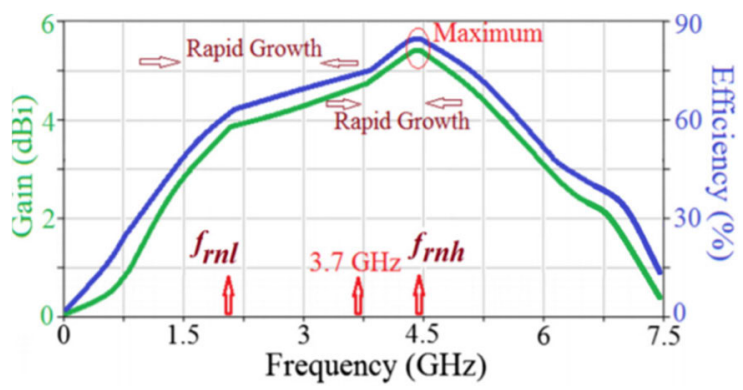

(d)

FIGURE 10. (a) Top surface of the proposed antenna, (b) its bottom side, (c) its reflection coefficient, and (its radiation gain and efficiency curves [54].

parameters of these antennas shown in Fig.11 confirm the developed concept.

\section{B. LEAKY-WAVE ANTENNAS (LWAS)}

New LWAs are one of the primary applications of the implementation of artificial metamaterial TL. Conventional elements whose main source of radiation is the power leakage propagating along the structure (this illustrates the term LW) leading to a high directivity are characterized as leaky-wave antennas [56], [57]. For leaky-wave antennas, the phase constant $\beta$ accounts for the radiated angle $\left(\theta_{m}\right)$ and the maximum beam width $(\Delta \theta)$ as follows [57]:

$$
\begin{aligned}
\sin \theta_{m} & \approx\left(\frac{\beta}{k_{0}}\right) \\
\Delta \theta & =\left(\frac{1}{\left(\frac{l}{\lambda_{0}}\right) \cos \theta_{m}}\right)
\end{aligned}
$$

$l$ is the antenna's length, $k_{0}$ and $\lambda_{0}$ are wave number and wavelength of the free-space, respectively. Note that radiation

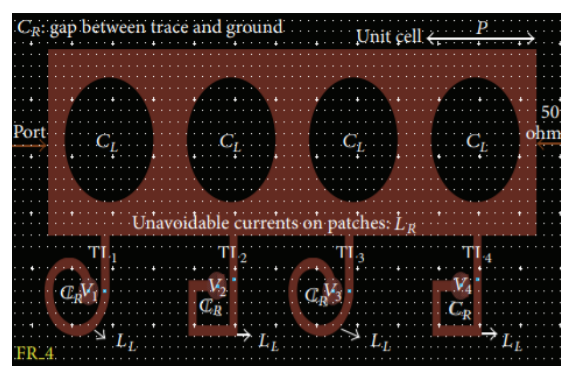

(a)

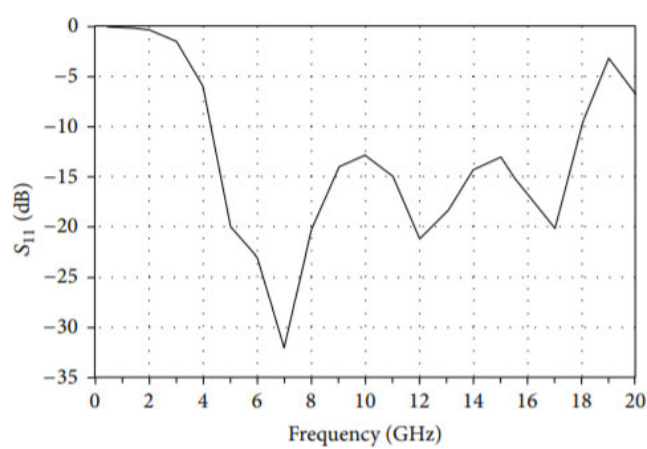

(b)

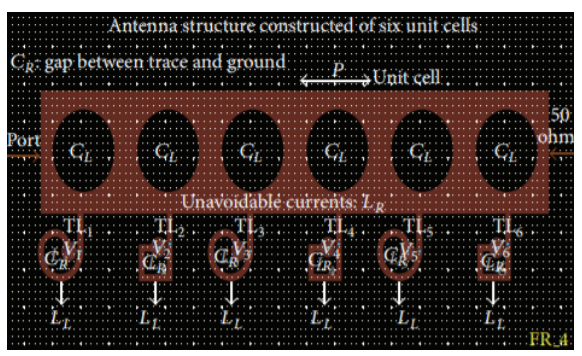

(c)

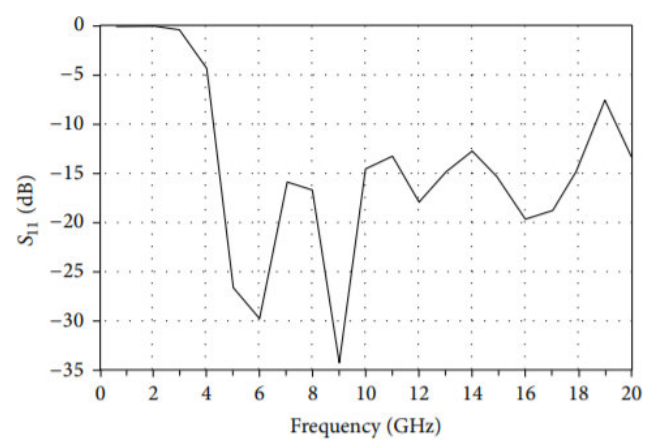

(d)

FIGURE 11. (a) Metamaterial-TL based antenna with four cells, (b) its reflection coefficient, (c) MTM-TL based antenna constructed of six-cells, (d) its reflection coefficient [55].

only occurs if $|\beta|<k_{0}$. When this condition holds, the structure tends to radiate, and the propagation constant (which is wave number of the free-space) accounts for the radiation beam angle. Since $\beta$ depends on the frequency dependent, frequency scanning is possible relative to the angle of the beam. For CRLH TLs, the propagation constant 
can be controlled to have a total frequency scanning comprising of radiation angle beams with positive (forward), zero (broadside), and even negative (backward. Experimentally, this is shown in [58]-[64]. In certain applications, it may not be feasible to use frequency scanning for signal generation. Consequently, frequency independent scanning and beam width control schemes are a necessity. Reconfigurable CRLH TL leaky-wave structures are a common means to achieve this. Typical structures allow for the control of the radiation beam at a fixed frequency by keeping it constant with respect to frequency. This approach is demonstrated in [61] where varactor diodes are applied. Other than varactor diodes, the implementation of metamaterial leaky-wave antennas by means of ferroelectrics and liquid crystals are also very promising [62]. In recent times, whole systems used for signal arrival detection arrival through the evaluation of the received power at the feed terminals have adopted this type of structure in their designs [63]. This approach allows for a simplification of the traditional systems which are mostly based on multiple antennas such as phase arrays. In this way, very similar functionalities are achieved using a single and compact radiating element at a lower cost and highly reduced power consumption. In [65] and [66] the tapered or modulated frequency-scanning LWAs have presented. These type of LWAs have applied to shape the scanned beam such as control of side-lobe level (SLL), confined beam shaping, near-field focusing/convergence, field divergence for sectorized shaped beams...etc. The drawback of these metamaterial-based CRLH TL LWAs is that, although they can balance the dispersion to obtain seamless backwardto-forward scanning, this balancing condition is in general more quite complicated to be obtained together with a taper design. In other words, to taper the LWA complex fields (both in amplitude and phase) it is required to modulate the LWA TL cross-section dimensions along the antenna long aperture. Doing this while keeping the balancing CRLH condition is quite complex [67].

According to existing literature, there are many variants and realizations of leaky-wave antennas. These include, but are not limited to concave and convex structures, dual-polarized or dual-band [64] LWAs, and others. More details on the review of these variants and realizations can be found in [68].

\section{1) BACKFIRE-TO-ENDFIRE LWA}

If there exists an optimum matching to the air impedance [69], the balanced CRLH TL can take the form of an efficient frequency-scanned LWA. However, some LWAs based on right-handed TLs operate with the fundamental mode, which has a leaky or fast-wave region $\left(|\beta|<k_{0}\right.$, where $k_{0}$ is the propagation constant of the free-space) and are not overmoded, and they have a direct feeding circuit without the need of creating CRLH TL [70]-[72]. They can also operate in both in only-forward-scanning (uniform) or periodic configuration (for backward-to-forward scanning). Similarly, conventional uniform LWAs can radiate through broadside direction by using a bidirectional configuration [73], [74]. Therefore, compared to the most of the conventional LW antennas, the CRLH-LW antenna has two distinct advantages. Firstly, a CRLH leaky-wave antenna can adequately function at its basic mode. This is because in the fundamental mode, a radiation (or fast-wave) region $\left(|\beta|<k_{0}\right)$ exists in addition to a guided (or slow-wave) region $\left(|\beta|>k_{0}\right)$ [2]. On the other hand, to make RH structures radiate, they must be implemented at higher order modes. Therefore, compared to their alternatives, they need a more complex and less-efficient feeding structure. This is mainly since the basic mode of the most of the RH-structures are inevitably guided $\left(\beta>k_{0}\right)$ and a typical CRLH-LW antenna can scan continuously from the backward (backfire) to forward (endfire) angles in contrast to the most of the traditional LW antennas. This can inferred through evaluating the LW antenna scanning angle relation stated as follows:

$$
\theta=\sin ^{-1}\left(\frac{\beta_{0}+2 n \pi / p}{k_{0}}\right)
$$

where $\beta_{0}, n$, and $p$ are the basic mode's propagation constant, the space harmonic [75], and the period, respectively. For a non-periodic LW-antenna, $n$ is null, and $\beta_{0}$ becomes operating mode's propagation constant. For the CRLH-LW antenna ( $n$ is null, i.e., the fundamental mode), $\theta$ assumes values ranging from $-90^{\circ}$ (backfire) to $+90^{\circ}$ (endfire), over the continuous frequency range where ||$\beta \mid<k_{0}$ holds [2]. Forward and backward scanning can be obtained by making the CRLH LW antenna operational below or above its transition frequency $\left(\omega_{0}\right)$, respectively. For the balanced CRLH-TL, at $\omega_{0}$ the antenna may have a broadside radiation since $v_{g} \neq 0$ at $\beta=0$. On the other hand, traditional non-periodic LW-antennas are only able to scan from broadside to endfire, because $\beta$ always assumes positive values. Additionally, broadside radiation is not feasible for conventional non-periodic LW-antennas. This is because for $\mathrm{RH}$ structures, $v_{g}$ is null (i.e., a standing wave) at a null value or position for $\beta$. By working at negative and positive space harmonics $(n= \pm 1, \pm 2, \pm 3, \ldots)$, conventional periodic LWAs are capable of scanning from backfire to endfire even though broadside radiation cannot occur [69].

To have backfire-to-endfire operations, a new leaky-wave antenna able of scanning from $-25^{\circ}$ to $+45^{\circ}$ is presented in [76]. The antenna in [76] adopts MTM-TLs and its physical implementation comprises of spiral and rectangular slots on a Monofilar Archimedean structure having metallic via-holes and spiral inductors. To realize the metamaterial antenna, the effects of left-handed capacitances in series are produced by the slots, and the effects of shunt left-handed inductances are produced by the spirals with via-holes. The physical implementation of the antenna's prototype is displayed in Fig.12. The prototype is implemented on a FR4 lossy substrate having an electrical dimensions of $0.0302 \lambda_{0} \times 0.0357 \lambda_{0} \times 0.0008 \lambda_{0}$, where $\lambda_{0}$ represents the wavelength of free-space at $0.165 \mathrm{GHz}$. The experimental 

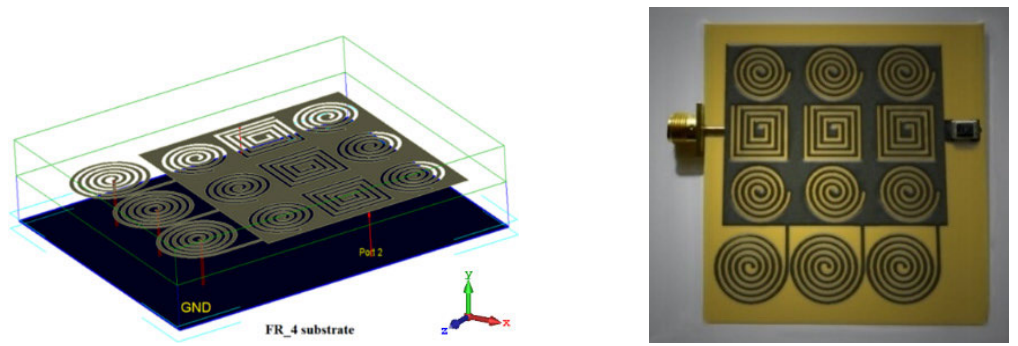

(a)

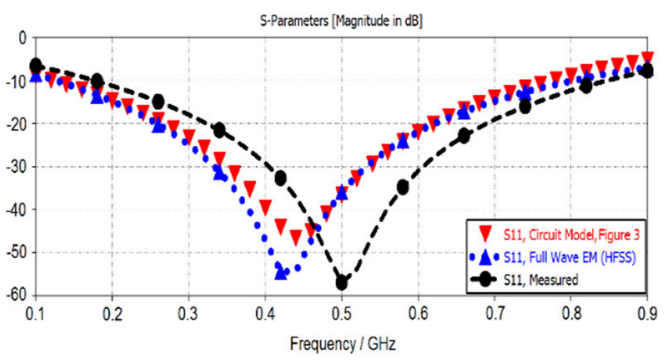

(b)

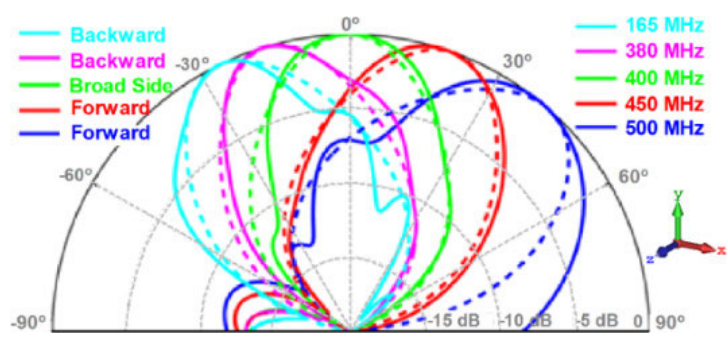

(c)

FIGURE 12. (a) Backfire-to-endfire LWA, (b) reflection coefficient responses, and (c) radiation patterns. HFSS: solid lines and experimental: dashed lines [76].

antenna's frequency bandwidth is $0.71 \mathrm{GHz}$ (i.e., $165 \mathrm{MHz}$ to $875 \mathrm{MHz}$ ) and this corresponds to $136.5 \%$ fractional bandwidth. The primary improvement of this antenna is its wide-angle scanning which ranges from $-25^{\circ}$ to $+45^{\circ}$ at desired gain of $1.2 \mathrm{dBi}$ and radiation efficiency of $50.1 \%$, respectively, both measured at an operating frequency of $400 \mathrm{MHz}$. The antenna's wide-angle scanning properties make it very promising suitable for passive radar (i.e., VHF-UHF bands) applications spanning across FM-Radio, television, and mobile phones.

In [31], an novel beam scanning LWA capable of wideangle scanning over a range from $-35^{\circ}$ to $+34.5^{\circ}$ between $57-62 \mathrm{GHz}$ and having broadside radiation centered at $60 \mathrm{GHz}$ is proposed and investigated using empirical results. The proposed LWA design is in [31] adopts the concept of CRLH-TL. Its structural single layer consists of a matrix of $3 \times 9$ square slits implemented on a dielectric layer. For bandwidth and radiation enhancement, $\Pi$ and T-formed slits have printed on the ground-plane (GND). The antenna shows good MTM property and provides beam scanning functionalities relative to the frequency using the matrix of square slots. The physical dimensions of the antenna is $18.7 \times 6 \times 1.6 \mathrm{~mm}^{3}$ and its electrical one is $3.43 \lambda_{0} \times 1.1 \lambda_{0} \times$ $0.29 \lambda_{0}$, where $\lambda_{0}$ represents the wavelength of the free space at $55 \mathrm{GHz}$. The antenna's impedance bandwidth is $10 \mathrm{GHz}$ (i.e., $55-65 \mathrm{GHz}$ ) corresponding $16.7 \%$ fractional bandwidth. At $62 \mathrm{GHz}$, its optimum gain is $7.8 \mathrm{dBi}$ and the efficiency is $84.2 \%$. The antenna has a lightweight and low profile making it inexpensive to mass produce. The configuration and performance parameters of the proposed LWA have depicted in Fig.13.
A innovative small structure constructing of six E-formed arms configured and laid out in two rows and three columns as depicted in Fig.14 is presented in [32]. The antenna structure in [32] is proposed as a new LWA capable of steering its beam in the broadside direction from $-30^{\circ}$ to $+15^{\circ}$. It is operational in over a experimental frequency bandwidth of $0.93-3.65 \mathrm{GHz}$ (that is about $119 \%$ for all frequency points where $S_{11}$ less than or equal to $-10 \mathrm{~dB}$ ). As the frequency increases, its scanned angle deflects from backward radiation to forward radiation. The antenna structure has a total phyical dimensions of $19.2 \mathrm{~mm} \times 15.2 \mathrm{~mm} \times 1.6 \mathrm{~mm}$ (i.e., an electricl dimensions of $0.059 \lambda_{0} \times 0.047 \lambda_{0} \times 0.004 \lambda_{0}$, where $\lambda_{0}$ represents the wavelength of the free space at $0.93 \mathrm{GHz}$ ). It is implemented on a $1.6 \mathrm{~mm}$ thick Rogers RT/Duroid 5880 layer having $\varepsilon_{r}=2.2$ and $\tan \delta=0.0009$. In the broadside direction, the maximum experimented gain is $8 \mathrm{dBi}$ and the radiation efficiency is $90 \%$. The antenna has a low-profile antenna making it to be easily flush-mounted on various components and structures such as mobile devices, cellular base stations and vehicles.

\section{2) TRAVELLING WAVE ANTENNA}

A single-layer travelling-wave antenna (TWA) based on CRLH MTM-TL structure is presented in [45]. The antenna is implemented via a combination of inter-digital capacitances and dual-spiral inductance slits. With incorporating dual-spiral inductance slits within the CRLH MTM-TL, a small TWA is achieved (see Fig.15). The dimension of the CRLH MTM-TL TWA is $21.5 \times 30.0 \mathrm{~mm}^{2}$ or $0.372 \lambda_{0} \times 0.520 \lambda_{0}$, where $\lambda_{0}$ is the wavelength of the 


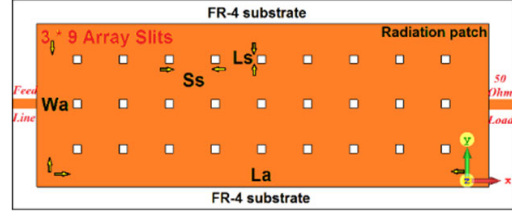

Simulated layout, top-view

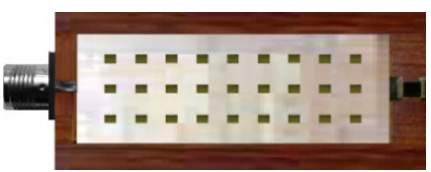

Manufactured prototype, top-view

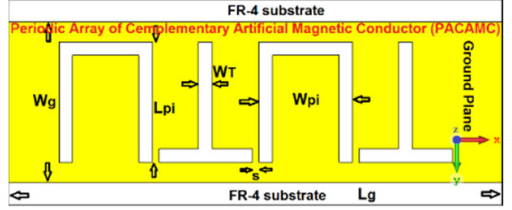

Simulated layout, bottom-view

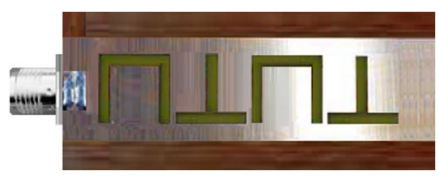

Manufactured prototype, bottom view

(a)

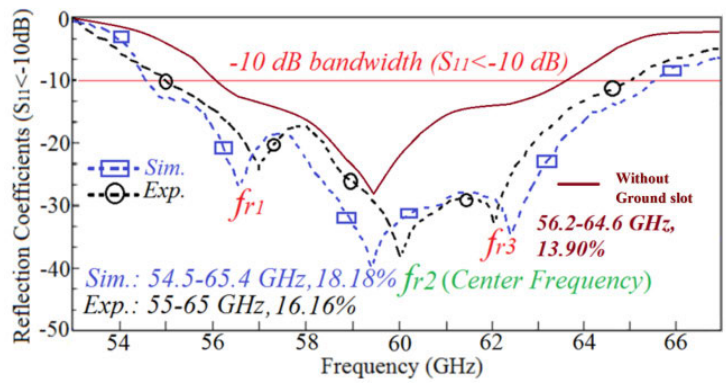

(b)
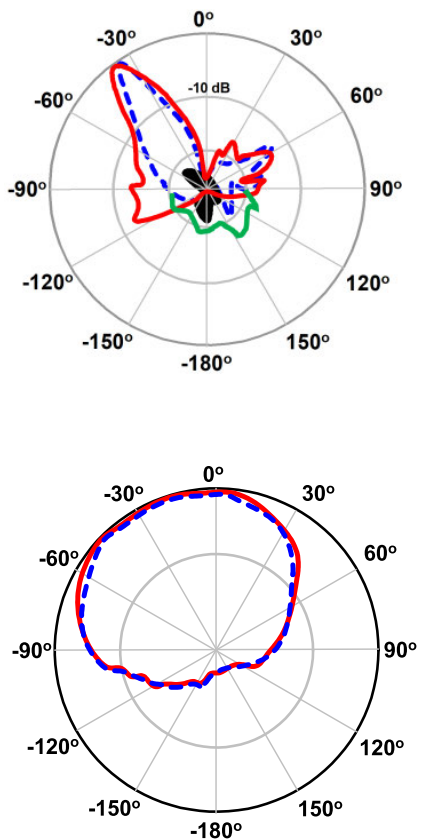

(a) $55 \mathrm{GHz}$

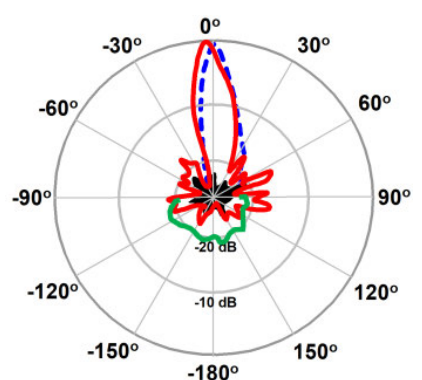

E-plane ( $x z$-plane)

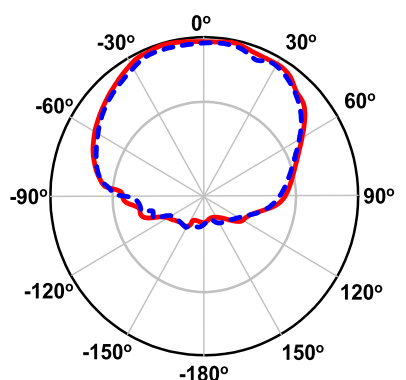

(a) $60 \mathrm{GHz}$

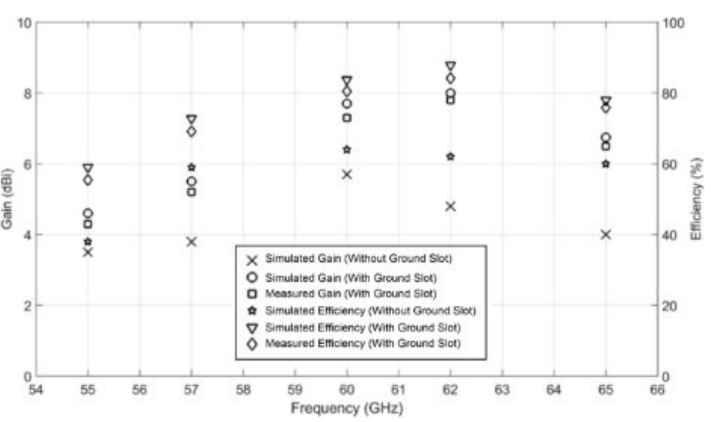

(c)
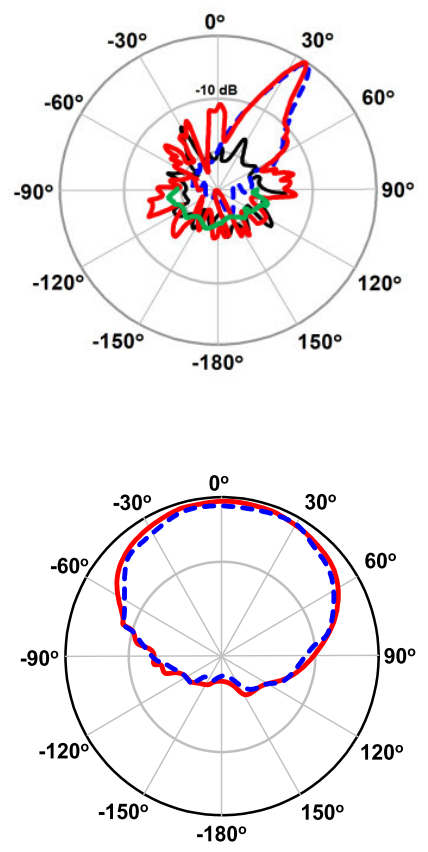

(a) $65 \mathrm{GHz}$

H-plane (yz-plane)

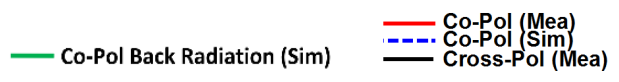

(d)

FIGURE 13. (a) Leaky-wave antenna, (b) reflection coefficient responses before and after implement the GND slots. Dashed lines plot the case after apply the slots, (c) radiation gain and efficiency responses when the antenna has unloaded and loaded with GND slots, and (d) backward to forward radiation patterns [31]. 

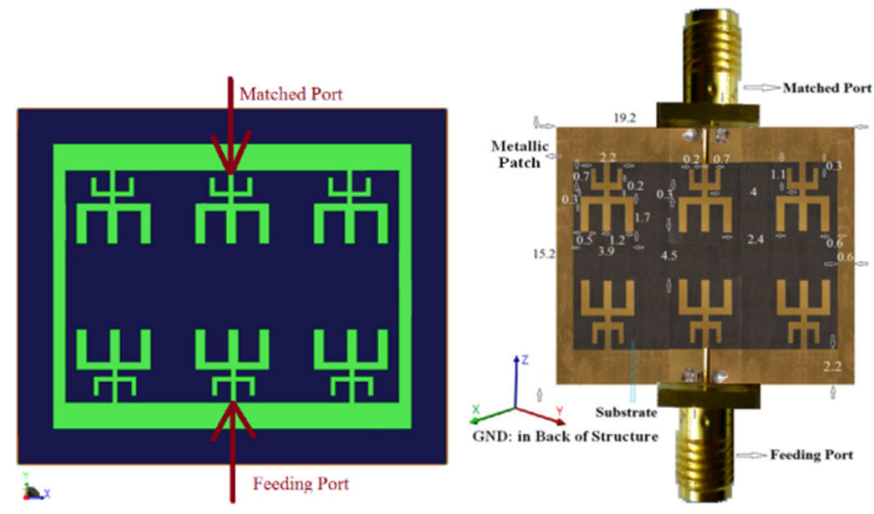

(a)

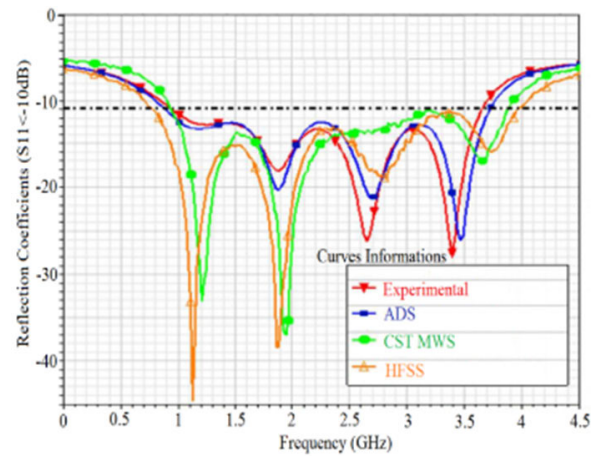

(b)

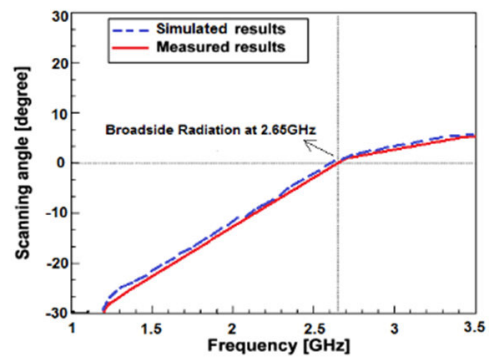

(d)

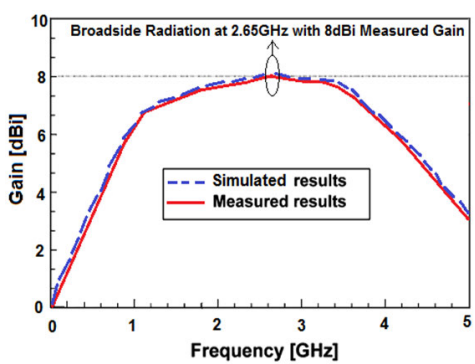

(e)

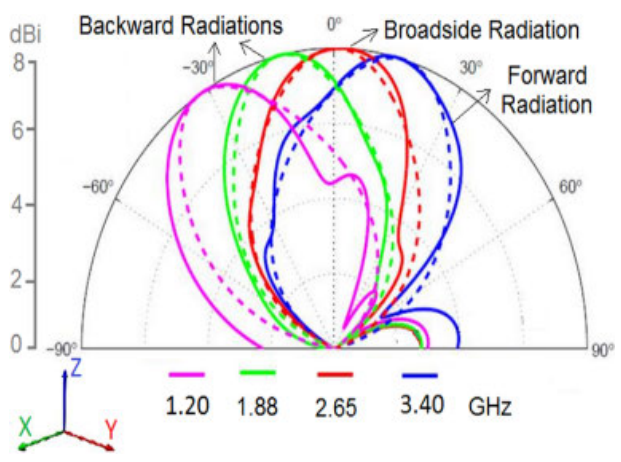

(c)

FIGURE 14. (a) Prototypes of the LWA (structural parameters have mentioned in millimeter), (b) reflection coefficients, (c) 2-D radiation patterns. Solid and dashed lines represents the measured and simulated results, respectively, (d) Scanning angle as function of the frequency, (e) Gains curves as function of the frequency, and (f) Gain curves versus scanning angle [32].

free-space at the center frequency of $5.2 \mathrm{GHz}$. The manufactured TWA works throughout $1.8-8.6 \mathrm{GHz}$ with a feasible bandwidth larger than $120 \%$. It shows a highest gain and radiation efficiency of $4.2 \mathrm{dBi}$ and $81 \%$ at $5 \mathrm{GHz}$. With preventing the utilize of lumped elements, via-holes, and defected ground structures (DGS), the TWA model is more economical for mass generation and simple to integration into wireless communication systems.

[46] introduces a left-handed MTM TWA based on MTM-TL to improve the antenna's gain and radiation efficiency without compromising on its practical bandwidth. The antenna shown in Fig.16 comprises of a series of coupled unit-cells having "X-formed" slits that are inductively terminated to GND. The antenna's effective aperture improves by enhancing the number of unit-cells. As a result, the gain improves and the radiation efficiency performance has no negative effect on the antenna's feasible bandwidth. The antenna's characterisation is carried out utilizing HFSS ${ }^{\mathrm{TM}}$, and it is manufactured applying standard PCB fabricating methods on a $1.6 \mathrm{~mm}$ thick dielectric layer with a $\varepsilon_{r}=2.2$. The antenna works within frequency band of 0.4-4.7 GHz. The antenna's electrical dimensions is $0.017 \lambda_{0} \times 0.006 \lambda_{0} \times 0.0020 \lambda_{0}$, where $\lambda_{0}$ is the wavelength of the free-space at $0.4 \mathrm{GHz}$. The antenna is considerably smaller compared with similar traditional designs. Optimum gain and radiation efficiency occurred at $2.5 \mathrm{GHz}$ are $2 \mathrm{dBi}$ and $65 \%$. These benefits make the antenna attractive for utilize in multiple wireless communication applications. 


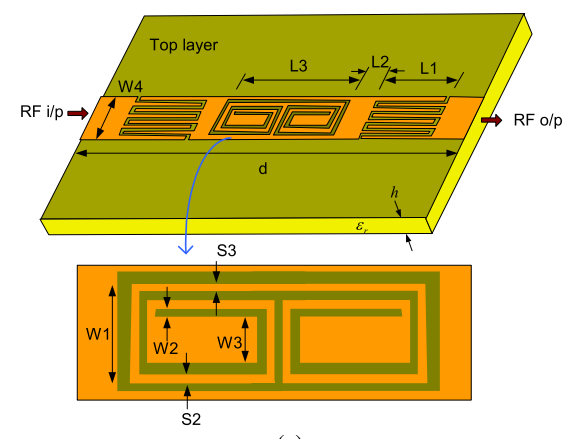

(a)

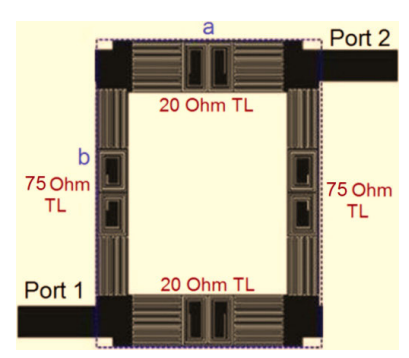

(b)

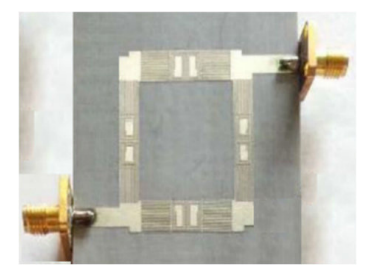

(c)

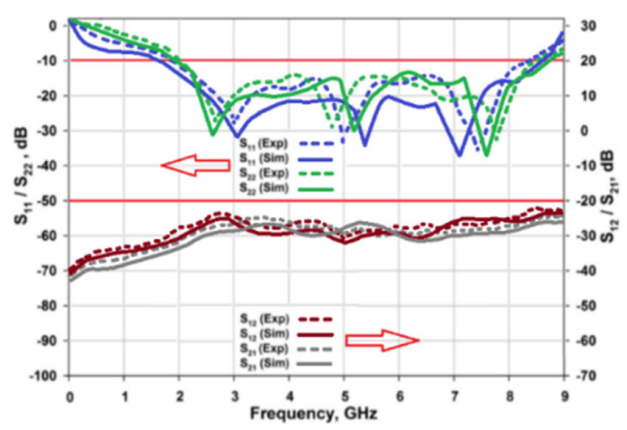

(d)

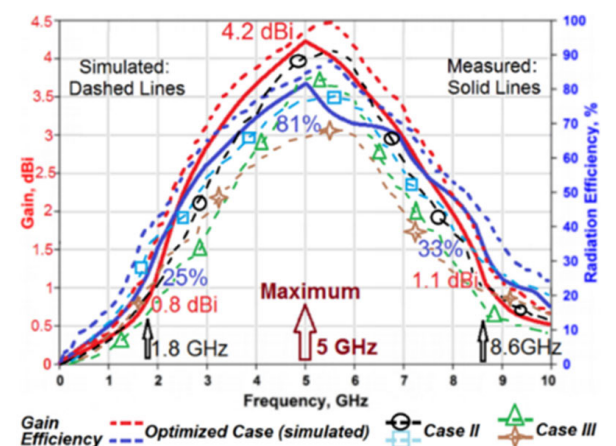

(e)

FIGURE 15. (a) CRLH MTM-TL implemented on 50 ohm microstrip line, (b) layout of the CRLH MTM-TL TWA, (c) manufactured prototype, (d) transmission and reflection coefficient responses of the TWA, and (e) TWA's gain and radiation efficiency responses [45].

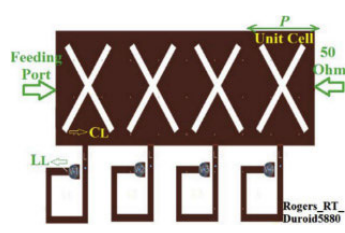

(a)

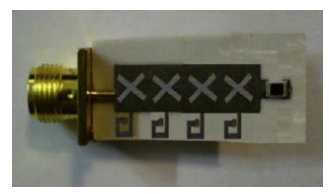

(c)

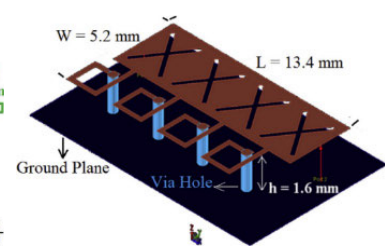

(b)

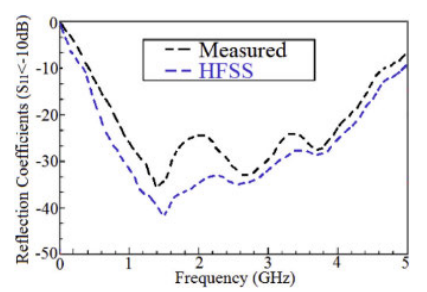

(d)
FIGURE 16. (a-c) Antenna's layout, and (d) its reflection coefficients responses [46].

\section{ZEROTH ORDER RESONATOR ANTENNA}

A unique property of CRLH metamaterials is that it is possible to obtain a null value for $\beta$ when the frequency is not equal to the zero. As shown in [33], a new zeroth order resonator (ZOR) can be generated based on this property. This type of resonator mainly comprises of a single unit cell (Fig.6) which is open-ended by capacitive slits. A $1.7 \mathrm{~mm}$ thick Rogers RT/Duroid 5880 layer with $\varepsilon_{r}=2.2$ is used for the implementation of the resonator. When $\beta$ assumes a null value, no phase shift occurs throughout the resonator. This is because the phase shift is defined as $\varphi=-\beta d=0$. Furthermore, the dependence of the resonance on only the reactive loadings and its non-relation to the length of the structure can be demonstrated.

As shown in [77], to build a ZOR antenna, the ZOR resonator earlier described can be adopted. For this construct, the microstrip-based unit-cell includes an inter-digital capacitance and a meandered-line shunted and linked to a rectangular patch which acts as a virtual GND. It is possible for the antenna's physical dimensions to be smaller compared with a half-wavelength because the physical dimensions of the ZOR do not affect the resonance. Therefore, the reactive loadings in the antenna's unit-cells define the antenna' physical dimensions. In [77], the reduction of size that can be achieved at design frequency of $4.88 \mathrm{GHz}$ with a ZOR antenna is demonstrated. The antenna has a size of $10 \mathrm{~mm}$, while the $\lambda / 2$ microstrip patch antenna's length is $20.6 \mathrm{~mm}$ when considering the same design frequency and substrate.

Novel concepts for the modelling of compact printed planar MTM antennas with special layouts for broad band $\mathrm{RF}$, microwave and wireless communication systems are introduced and investigated in [78]. To implement the antennas, CRLH-TLs (i.e., general TLs containing both LH and $\mathrm{RH}$ characteristics) are realized by the standard fabrication methods. In this way, the E-formed slots and the SRR acting as spiral inductances constitute the series LH capacitances 


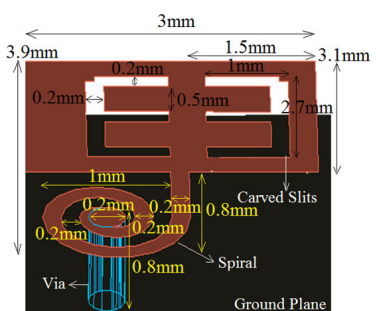

(a)

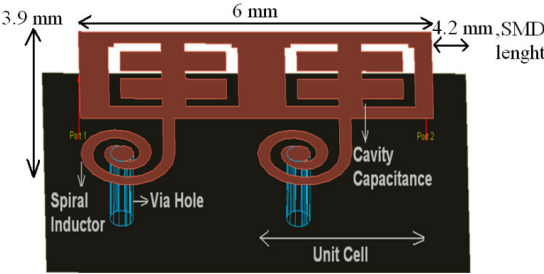

(b)

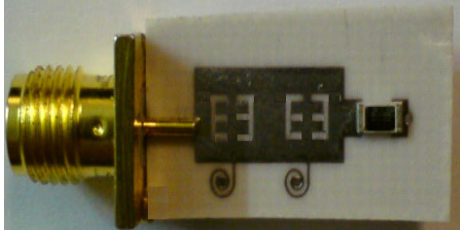

(c)

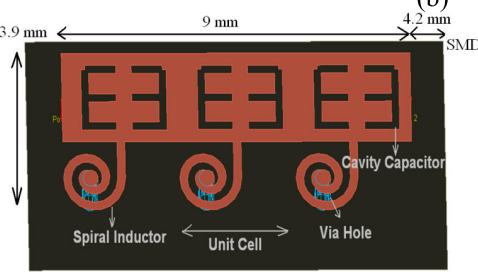

(d)

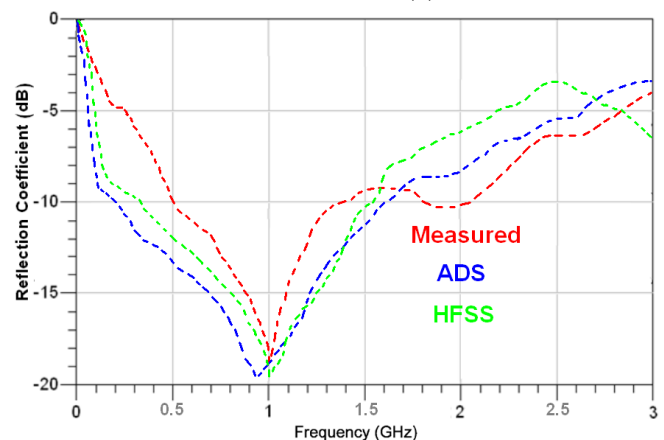

(f)

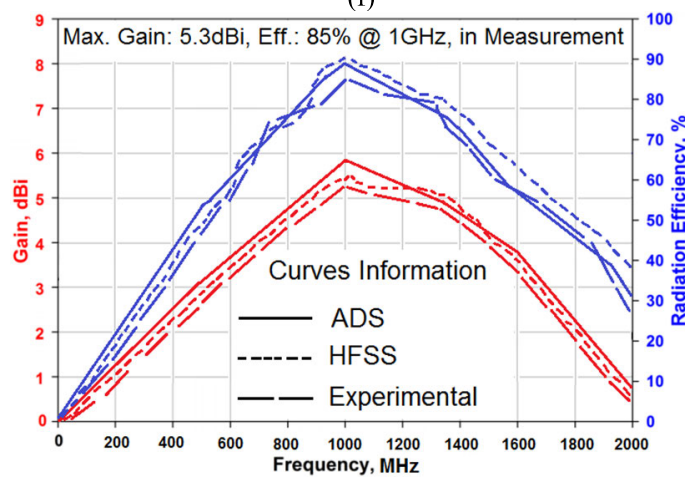

(h)

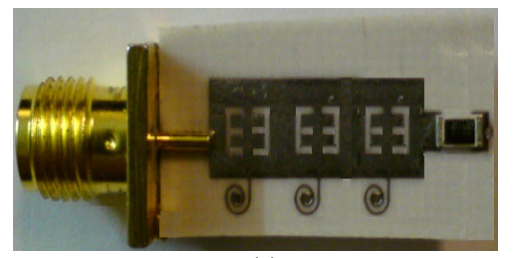

(e)

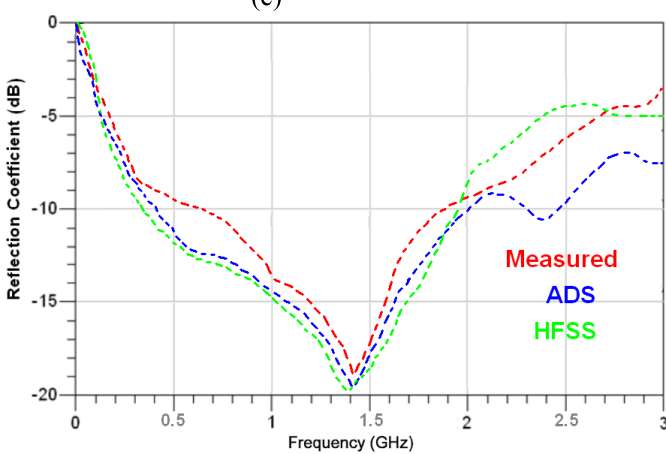

(g)

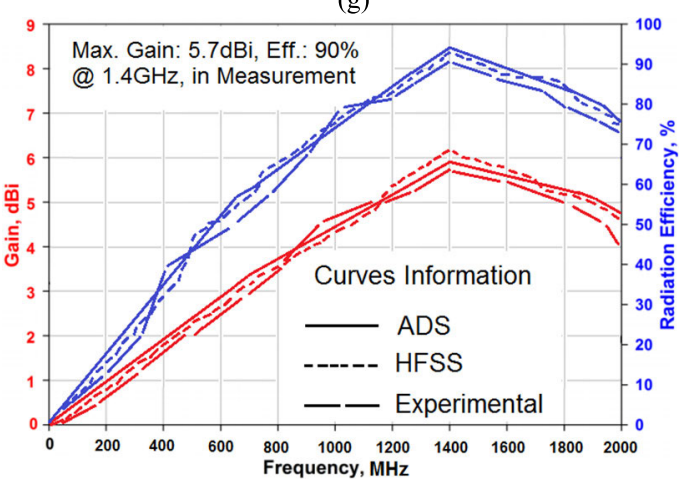

(i)

FIGURE 17. (a) Proposed unit cell layout of the resonating MTM based miniaturized antennas, (b, c) E-formed antenna constructed of two cells, (d, e) E-formed antenna constructed of three cells, (f, g) reflection coefficients responses for first and second antennas, respectively, $(h, i)$ gain and radiation efficiency curves for first and second antennas [78].

$\left(\mathrm{C}_{\mathrm{L}}\right)$ and shunt $\mathrm{LH}$ inductances $\left(\mathrm{L}_{\mathrm{L}}\right)$, respectively. By optimizing the quantities and dimensions of these elements, good operational performances can be obtained for the antennas. Fig.17 shows that the proposed antennas are fabricated by applying two and three unit-cells with E-shaped configurations possessing total size of $0.017 \lambda_{0} \times 0.006 \lambda_{0} \times 0.001 \lambda_{0}$ and $0.028 \lambda_{0} \times 0.008 \lambda_{0} \times 0.001 \lambda_{0}$, respectively, where $\lambda_{0}$ represents the wavelength of the free-space at the operating frequencies of $500 \mathrm{MHz}$ and $650 \mathrm{MHz}$, respectively. The antennas support the frequency bandwidths of $0.5-1.35 \mathrm{GHz}$ $(0.85 \mathrm{MHz})$ and $0.65-1.85 \mathrm{GHz}(1.2 \mathrm{GHz})$, which translates to $91.9 \%$ and $96.0 \%$ fractional bandwidths, respectively.
Besides the small size and broad bandwidth properties, the experimented gains and efficiencies for the fisrt and second antennas are $5.3 \mathrm{dBi}$ and $85 \%$ happened at $1 \mathrm{GHz}$, and $5.7 \mathrm{dBi}$ and $90 \%$ occurred at $1.4 \mathrm{GHz}$, respectively.

\section{SLOTTED ANTENNA}

A simple approach to expand the aperture of CRLH-MTM antennas with negligible effect on the its size has been presented [34]. Unlike most CRLH-based antennas, the antenna in [34] is via-less. It is exhibited to operate over a broad band covering the UHF- and C- bands with proper radiation specifications. The antenna configuration shown 


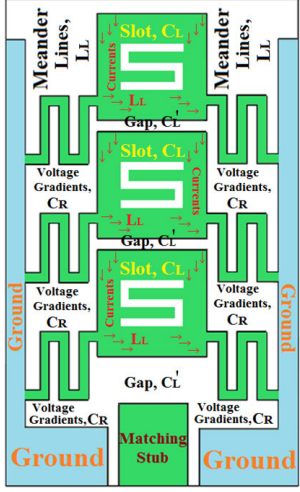

top-view

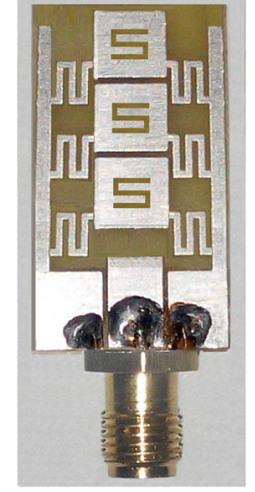

top-view

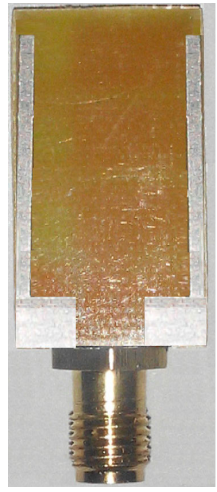

back-view (GND)

(a)

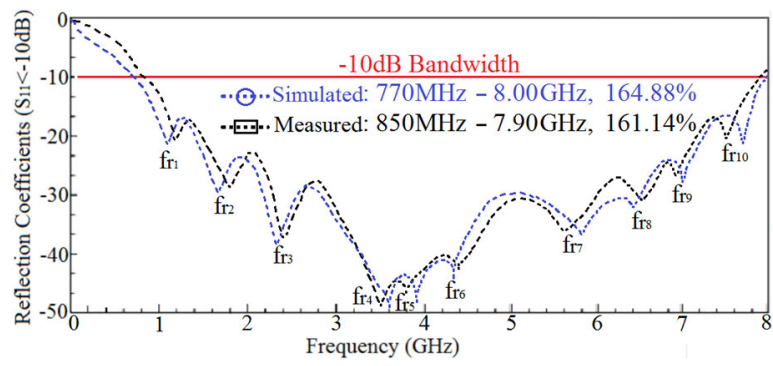

(b)

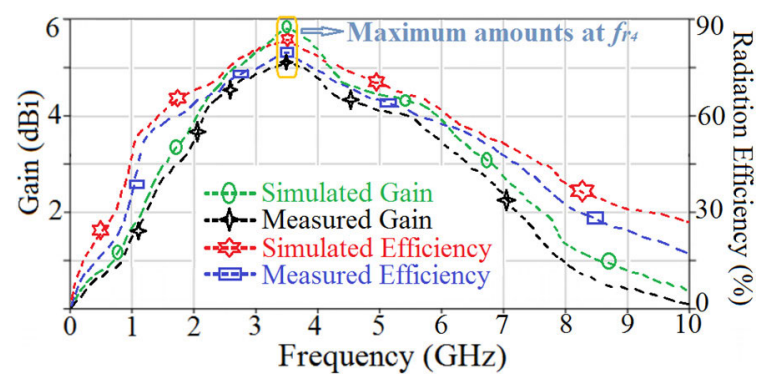

(e)

FIGURE 18. (a) Extended CRLH MTM antenna, (b) its reflection coefficient responses, and (c) its radiation gain and efficiency curves [34].

in Fig.18 includes of vertically stacked CRLH unit-cells containing of a patch and meandered-lines. The patch has etched with an S-formed slit. The design applies a small GND space. The meandered-line inductor is grounded utilizing CPW ground. This eliminates the use of a conventional CRLH TL metal on GND. The antenna is fed by a CPW match stub which is electromagnetically coupled to the unitcells. Its dimensions are $17.5 \mathrm{~mm} \times 32.15 \mathrm{~mm} \times 1.6 \mathrm{~mm}$, which corresponds to $0.204 \lambda_{0} \times 0.375 \lambda_{0} \times 0.018 \lambda_{0}$ where $\lambda_{0}$ represents the wavelength of the free-space and it is equal to 3.5 GHz. By carrying out a parametric study, the antenna's performance is optimized in terms of frequency bandwidth, radiation gain and efficiency. The results approve that, the antenna operates within frequency band of $0.85-7.90 \mathrm{GHz}$, which is related to a feasible bandwidth of $161.14 \%$. The antenna's highest radiation gain and efficiency happened at $3.5 \mathrm{GHz}$ are $5.12 \mathrm{dBi}$ and $\sim 80 \%$, respectively.

The design feasibility of a MTM antenna structure for multi-octave band operation is described in [35]. The MTM unit-cell (Fig.19) contains of an L-formed slot that is printed within a rectangular patch including a grounded inductive spiral. The slot fundamentally acts as a series LH-capacitor and the spiral behaves as a shunt LH-inductor. The antenna is designed and optimized for frequency bandwidth, radiation gain and efficiency performances applying the CST Microwave Studio. The antenna shows a frequency bandwidth of $6.02 \mathrm{GHz}$, which is related to a feasible bandwidth of $172.49 \%$ that is higher than what is obtainable in multiband antennas in literature. The antenna depicts a optimum gain and radiation efficiency of $3.7 \mathrm{dBi}$ and $73 \%$ occurred at $3.25 \mathrm{GHz}$. The antenna' physical footprint area is comparable to other broadband antennas available in literature. The total size of the antenna at $0.48 \mathrm{GHz}$ and $3.25 \mathrm{GHz}$ is $0.037 \lambda_{0} \times 0.027 \lambda_{0} \times 0.002 \lambda_{0}$ and $0.25 \lambda_{0} \times 0.18 \lambda_{0} \times 0.017 \lambda_{0}$, where $\lambda_{0}$ is the wavelength of the free-space.

New planar slotted-antennas have proposed in [36] that display proper radiation properties at the UHF-SHF bands. The antennas (Fig.20) have implemented by realizing metamaterial unit-cells modelled via capacitive slits printed inside the radiating patch and grounded spiral-formed inductive stubs. The proposed designs have manufactured on the Rogers RO4003 layer with $\varepsilon_{r}=3.38$ and thickness of $1.6 \mathrm{~mm}$. The first antenna consists of five symmetrical unit-cells possessing a slot-inductor-slot layout. It works throughout a broad bandwidth of $1-4.2 \mathrm{GHz}$ with a highest gain and efficiency of $1.5 \mathrm{dBi}$ and $35 \%$ at $2 \mathrm{GHz}$. Second antenna contains of ten asymmetrical unit-cells with a slot-inductor formation realized on the same space of layer as the first design. Its gain is enhanced by $2 \mathrm{~dB}$ and its efficiency is improved by $25 \%$ and it works throughout $750 \mathrm{MHz}$ to $4.5 \mathrm{GHz}$. The asymmetrical unit-cell effectively extends the antenna's aperture without comprising its size. The antenna's 

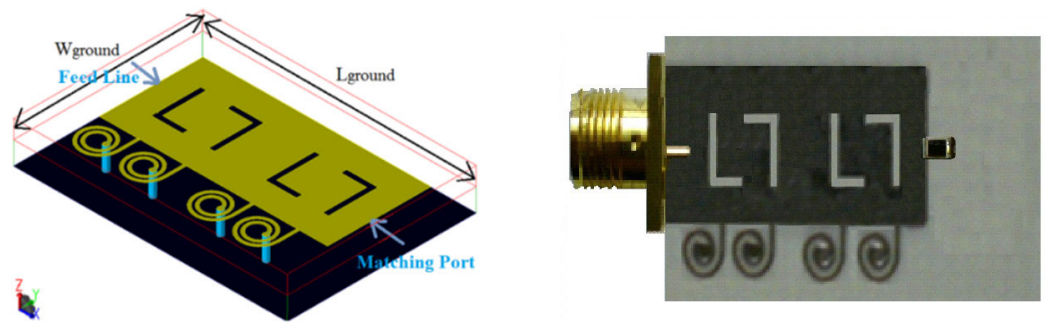

(a)

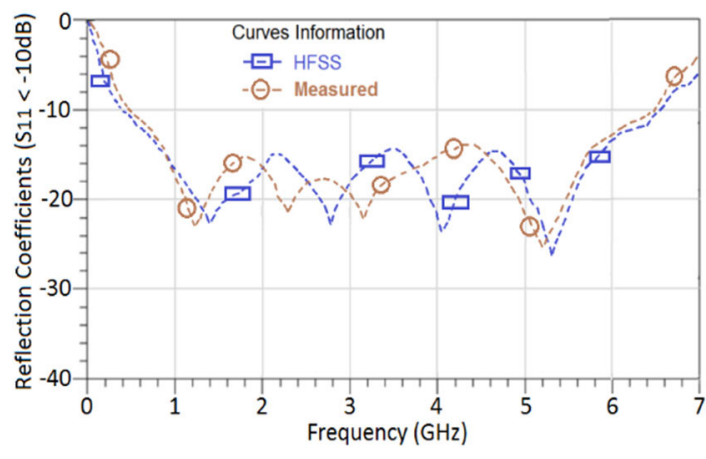

(b)

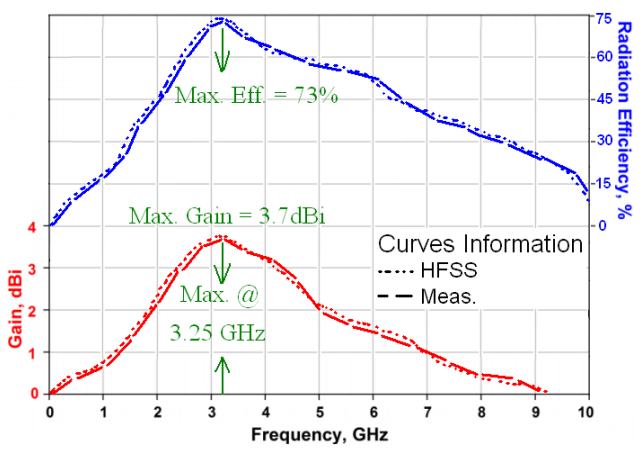

(c)

FIGURE 19. (a) Proposed metamaterial based antenna, (b) reflection-coefficient responses, and (c) gain and radiation efficiency curves [35].

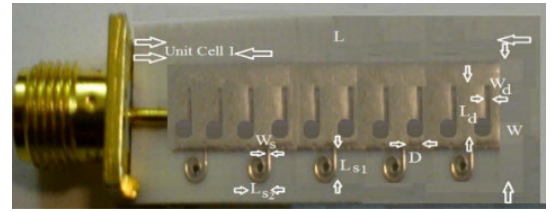

Antenna \#1

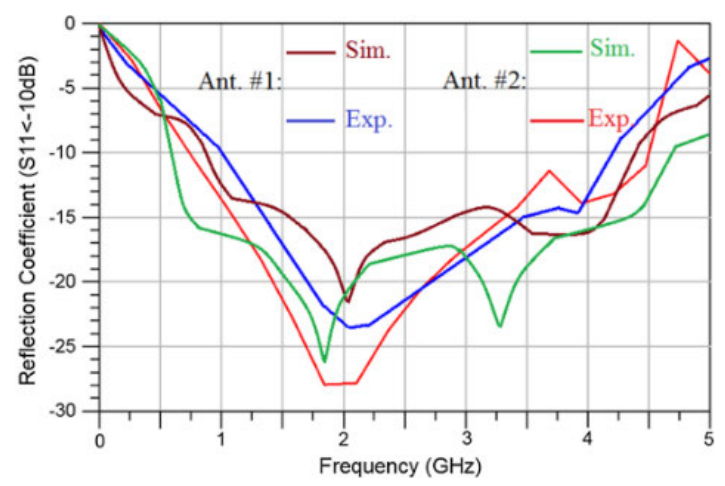

(b)

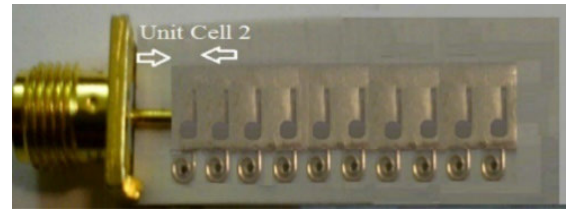

Antenna\#2

(a)

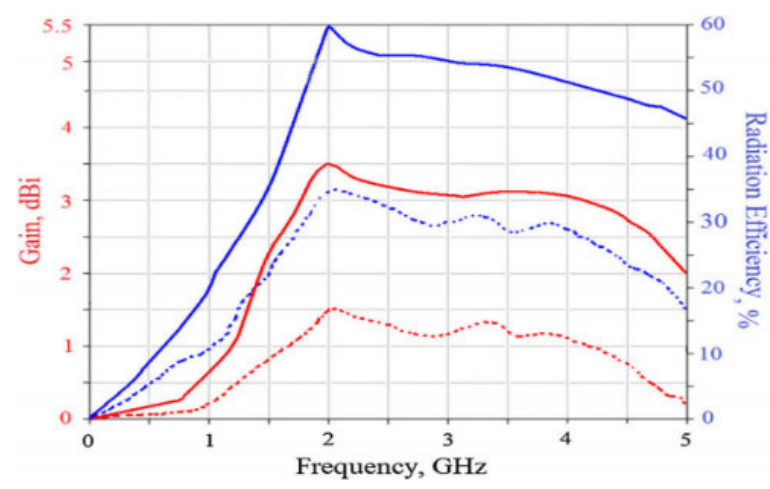

(c)

FIGURE 20. (a) Two planar slotted antennas, (b) reflection-coefficient responses, (c) gain and efficiency performances. (Ant.\#1: Dashed-lines, and Ant.\#2: Solid-lines) [36].

electrical size is $0.083 \lambda_{0} \times 0.033 \lambda_{0} \times 0.005 \lambda_{0}$, where $\lambda_{0}$ represents the wavelength of the free-space at $1 \mathrm{GHz}$.

The experimental data of a low-profile light-weight antenna based on a periodic array of the complementary artificial magnetic conductor MTM structure, which is constructed through loading the antenna with E-formed slots and inductive microstrip lines grounded applying metallic via-holes are presented in [37]. The antenna's prototype is shown in Fig.21 and it works throughout a broadband of $0.41-4.1 \mathrm{GHz}$, which relates to a feasible 


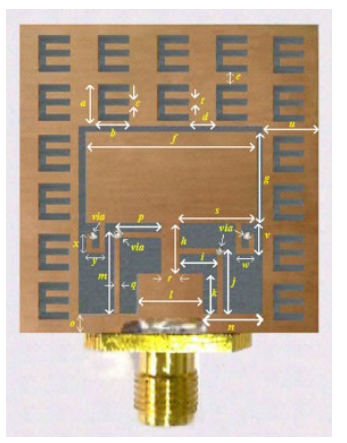

(a)

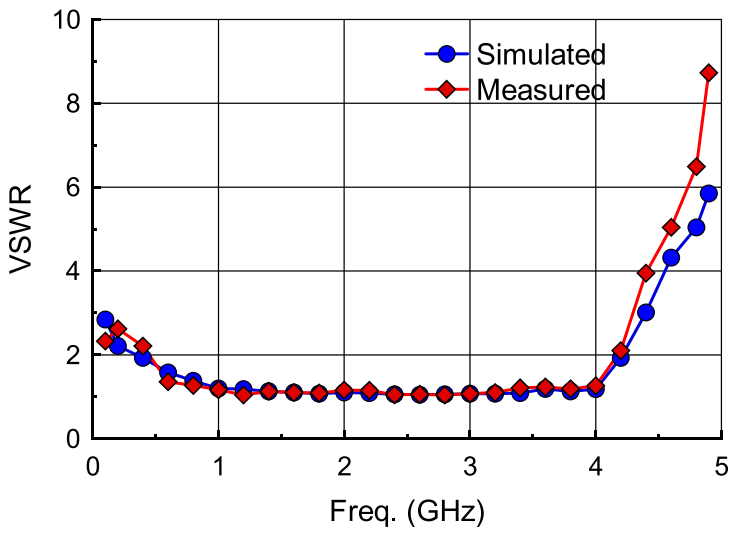

(c)

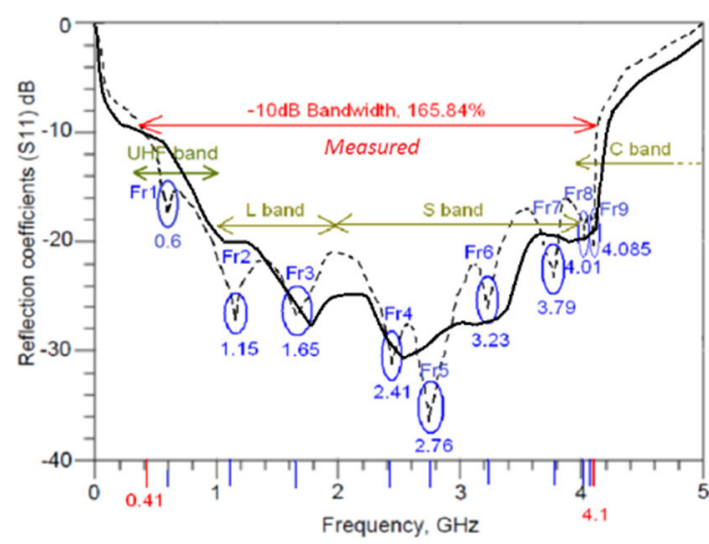

(b)

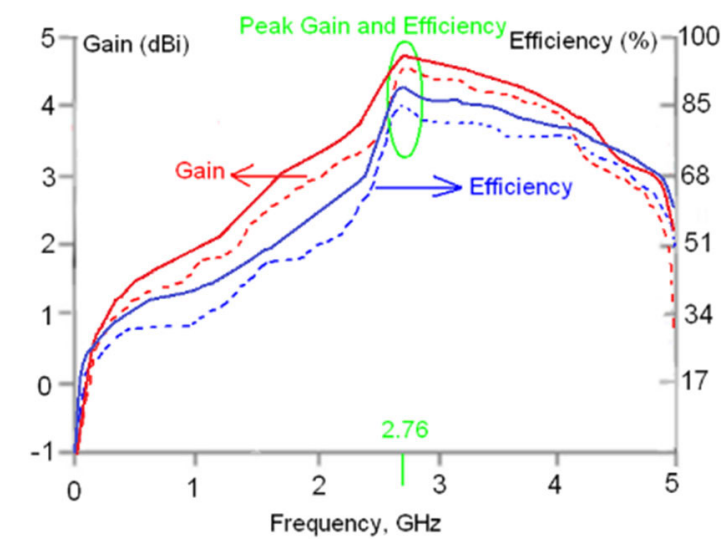

(d)

FIGURE 21. (a) Antenna, (b) reflection-coefficient responses, (c) voltage standing wave ratio response, and (d) gain and efficiency responses [37]. Simulated and measured results are presented by solid and dashed lines, respectively.

bandwidth of $165.84 \%$. The size is $40 \times 35 \times 1.6 \mathrm{~mm}^{3}$ or $0.054 \lambda_{0} \times 0.047 \lambda_{0} \times 0.0021 \lambda_{0}$, where $\lambda_{0}$ represents the wavelength of the free-space at $0.41 \mathrm{GHz}$. The highest gain and radiation efficiency of the optimized antenna occurred at $2.76 \mathrm{GHz}$ are $4.45 \mathrm{dBi}$ and $85.8 \%$, respectively. At the lower working band of $0.41 \mathrm{GHz}$, the antenna provides a gain and radiation efficiency of $1.05 \mathrm{dBi}$ and $32.5 \%$. The planar nature of antenna allows for a simple integration with wireless transceivers.

A new UWB small integrated antenna based on CRLH MTM-TLs has proposed and illustrated in [38]. The antenna structure is displayed in Fig.22 and it has implemented applying new inductance and capacitance components (i.e., the spiral and rectangular inductances) connected to the ground plane through the metallic via-holes. The etched $\mathrm{L}$ - and $\mathrm{T}$ - formed slots are designed by means of the MTM and standard manufacturing techniques on a PCB to realize shunt left-handed inductances $\left(L_{L}\right)$ and series left-handed capacitances $\left(C_{L}\right)$, respectively. By using the proposed approaches to realize the aforesaid components and using properly adjusted dimensions, the favorable characteristics are obtained. The antenna's physical dimensions is
$22.6 \times 5.8 \times 0.8 \mathrm{~mm}^{3}$ or $0.037 \lambda_{0} \times 0.009 \lambda_{0} \times 0.001 \lambda_{0}$, where $\lambda_{0}$ is the wavelength of the free-space at $0.5 \mathrm{GHz}$. The antenna works from $0.5-11.3 \mathrm{GHz}$, which is related to a practical bandwidth of $183 \%$. The radiation gains and efficiencies various frequencies of at $0.5,3,5,8$ and $11.3 \mathrm{GHz}$ are $1.5 \mathrm{dBi}$ and $20 \%, 3.4 \mathrm{dBi}$ and $45 \%, 4.8 \mathrm{dBi}$ and $57 \%$, $6.5 \mathrm{dBi}$ and $88 \%$, and $5.7 \mathrm{dBi}$ and $73 \%$, respectively. The significant advantages of the manufactured antenna include, but are not limited to unidirectional radiation patterns, cost effective, lightweight, low-profile, and compatibility and ease of integration within electronic systems. Results show that the proposed antenna can be fitted on communication systems and integrated into RF electronics to support today's multi band wireless application requirements requiring single or multiple feed designs to omit the need for antenna switches. Thus, the proposed antenna is a good potential nomine for modern industrial electronics applications.

[39] presents a new compact UWB antenna (see Fig.23) based on CRLH-MTM unit-cells for modern wireless communication systems. The physical dimensions of miniaturized antenna is $15 \mathrm{~mm} \times 7.87 \mathrm{~mm} \times 1.6 \mathrm{~mm}$ or $0.15 \lambda_{0} \times$ 


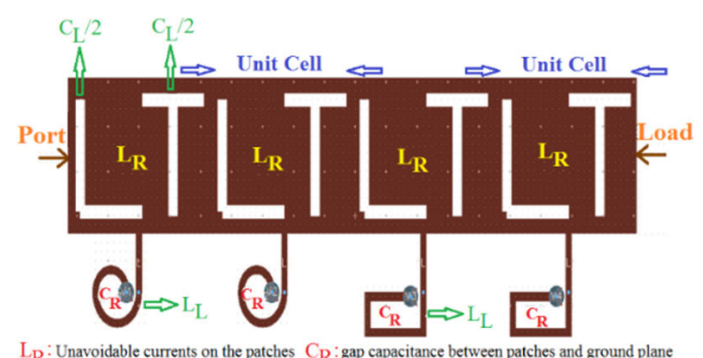

$\mathrm{L}_{\mathrm{R}}$ : Unavoidable currents on the patches $\mathrm{C}_{\mathrm{R}}$ : gap capacitance between patches and ground plane

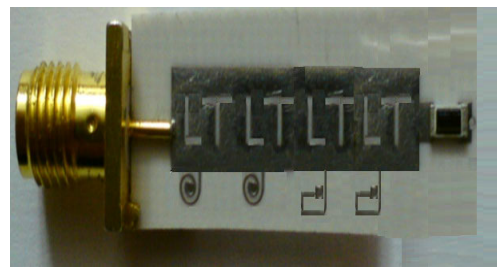

(b)

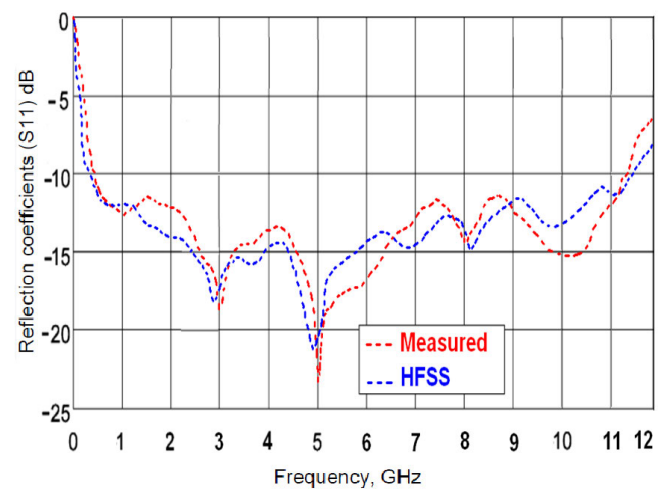

(d)

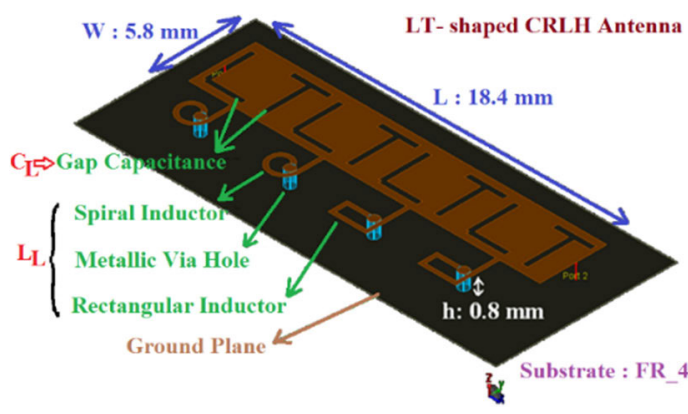

(a)

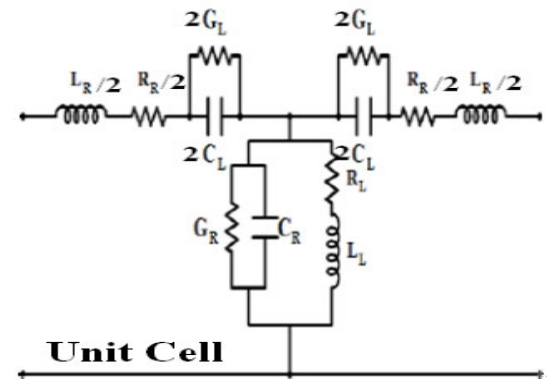

(c)

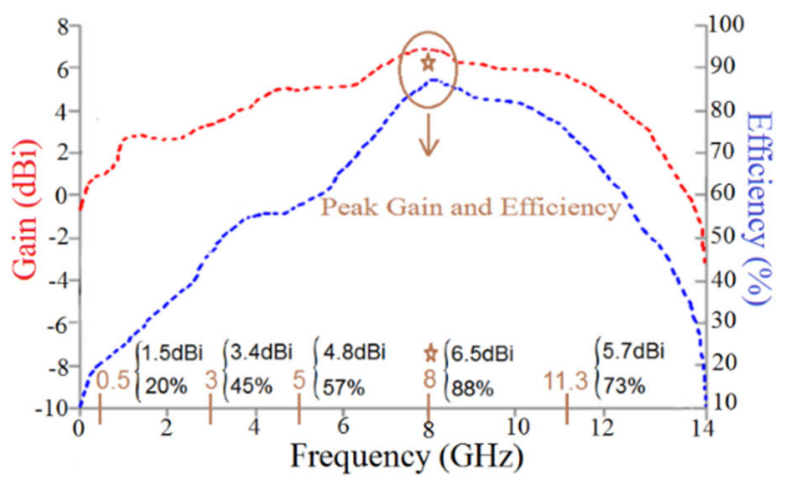

(e)

FIGURE 22. (a) CRLH antenna, (b) its manufactiured prototype, (c) unit cell' circuit model, (d) reflection coefficients, and (e) radiation gain and efficiency [38].

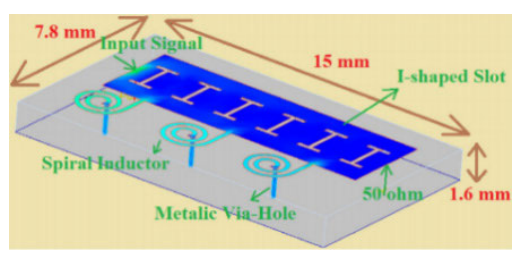

(a)

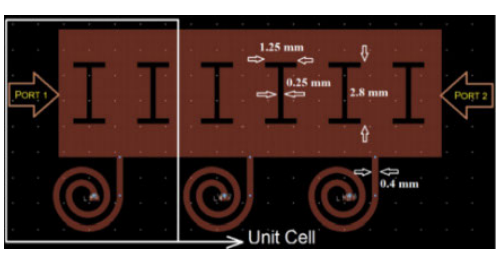

(b)

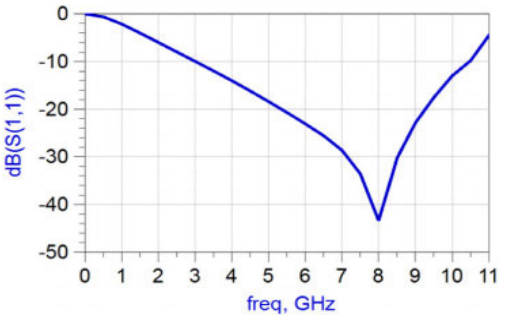

(c)

FIGURE 23. UWB MTM antenna with its return loss parameter [39].

$0.07 \lambda_{0} \times 0.01 \lambda_{0}$ where $\lambda_{0}$ is the free-space wavelength at $3 \mathrm{GHz}$. The antenna works over an frequency band of $3 \mathrm{GHz}$ to $10.6 \mathrm{GHz}$ that is corresponded to a practical bandwidth of $111 \%$. Gain and efficiency are more than $2.89 \mathrm{dBi}$ and $38.54 \%$, with the optimum values of $9.41 \mathrm{dBi}$ and $99.93 \%$. The antenna' specifications have accredited by the experimental data achieved from a manufactured prototype to make the proof of concept. 


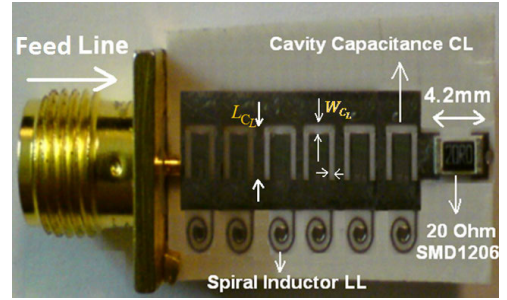

(a)

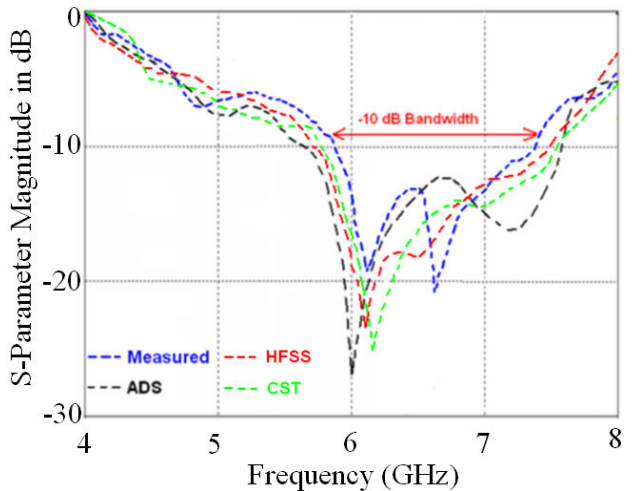

(c)

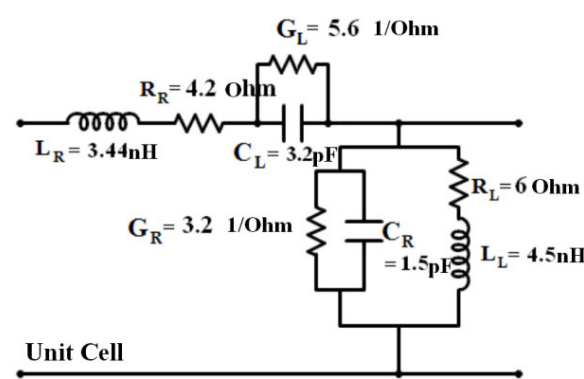

(b)

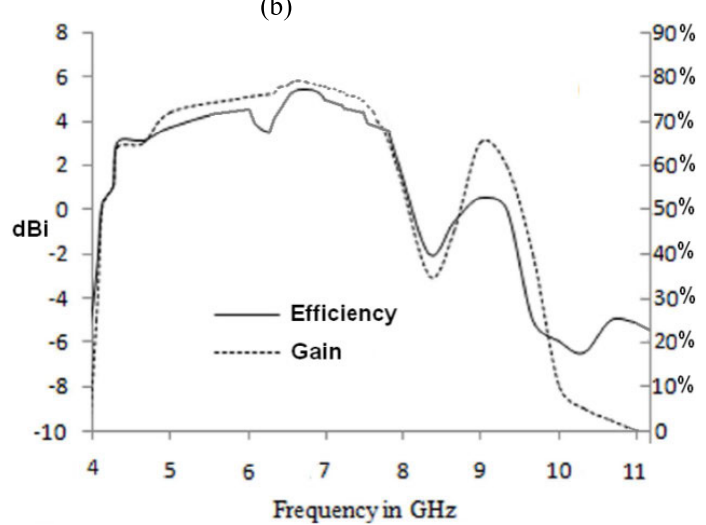

(d)

FIGURE 24. (a) MTM antenna, (b) unit-cell's circuit model, (c) reflection-coefficients, and (d) gain and radiation efficiency [40].

A innovative antenna based on CRLH-TH unit-cells realized applying $\pi$-shaped slits and spiral inductances that are engraved directly on the dielectric layer by standard fabrication approaches has been proposed in [40]. From Fig.24, it is clear that, each antenna's unit cell consists of a $\pi$-formed slits and a spiral inductor short-circuited to ground using a via-hole to have the series LH-capacitances $\left(C_{L}\right)$ and the shunt LH-inductances $\left(L_{L}\right)$, respectively. The antenna is moddeled to work over 5.8-7.3 GHz, which relates to a practical bandwidth of $23 \%$. The results approve the antenna shows a relatively broad bandwidth, high radiation gain and efficiency properties. The gain and efficiency at $6.6 \mathrm{GHz}$ are $4.8 \mathrm{dBi}$ and $78 \%$. Its unidirectional radiation pattern with $3 \mathrm{~dB}$ angular beamwidth of $90^{\circ}$ is constant throughout its working frequency range. The manufactured antenna is highly small and its physical dimensions in terms of the free-space wavelength at $5.8 \mathrm{GHz}$ is $0.39 \lambda_{0} \times 0.13 \lambda_{0} \times$ $0.015 \lambda_{0}$.

[41] investigates a small broadband antenna applying CRLH-TL MTM. The antenna has a practical bandwidth of $100 \%$ and it has been modelled to work in a large frequency band from $0.8-2.40 \mathrm{GHz}$ (see Fig.25). The antenna is built by realizing two CRLH-TL unit-cells including two inverted T-formed slits. The slits participate towards producing the series LH-capacitor $\left(C_{L}\right)$. The rectangular patch on which the slits are etched is grounded with spiral formed high impedance stubs that participate towards $\mathrm{LH}$ inductance $\left(L_{L}\right)$. The antenna's dimension is $14 \times 6 \times 1.6 \mathrm{~mm}^{3}$ (i.e., $0.037 \lambda_{0} \times 0.016 \lambda_{0} \times 0.004 \lambda_{0}$, where $\lambda_{0}$ is the wavelength of the free space at $0.8 \mathrm{GHz}$ ). The optimum gain and efficiency happened at $1.6 \mathrm{GHz}$ are $1.5 \mathrm{dBi}$ and approximately $75 \%$, respectively. The antenna is proper for utilize in wireless systems operating at UHF-, L-, S- bands, in particular, AMPS, GSM, WCDMA, UMTS, PCS, cellular, DCS, IMT-2000, JCDMA, KPCS, GPS, lower band of WiMAX.

In [42], metamaterial unit-cells are applied to model, develop and investigate two planar antennas. As shown in Fig.26, slits which are both H-formed or T-formed alongside a grounded spiral constitute the structural topology of the antenna. Series left-handed capacitance $\left(C_{L}\right)$ effects are produced by the slits and shunt left-handed inductance $\left(L_{L}\right)$ effects are produced by the spiral. Full-wave electromagnetic simulation tools have been used for the modelling, characterization and optimization of the unit-cell. Both the E-formed and $\mathrm{H}$-formed slot configuration of the antenna employ two unit-cells implemented on a $0.8 \mathrm{~mm}$ thick Rogers RO4003 layer having an $\boldsymbol{\varepsilon}_{r}$ of 3.38. The dimension of the $\mathrm{H}$-formed unit-cell antenna is $15 \times 6.9 \times 0.8 \mathrm{~mm}^{3}$ or $0.06 \lambda_{0} \times 0.02 \lambda_{0} \times 0.003 \lambda_{0}$, where $\lambda_{0}$ is the wavelength of the free-space at $1.2 \mathrm{GHz}$. The size of the T-formed unit-cell antenna is $15.5 \mathrm{~mm} \times 6.9 \mathrm{~mm} \times 0.8 \mathrm{~mm}$ or $0.05 \lambda_{0} \times 0.02 \lambda_{0} \times$ $0.002 \lambda_{0}$, where $\lambda_{0}$ is the wavelength of the free-space at $1.1 \mathrm{GHz}$. Fig. 26 shows the measurement results confirming that the $\mathrm{H}$-formed unit-cell antenna supports a working bandwidth of 1.2-6.7 GHz, relating to a practical bandwidth of $\sim 140 \%$. Fig. 26 also shows that the T-formed unit-cell antennas has an operational bandwidth of 1.1-6.85 GHz, corresponding to a practical bandwidth of $\sim 145 \%$. Over its 


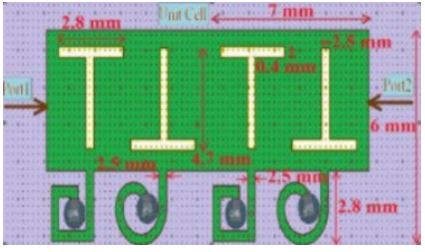

(a)

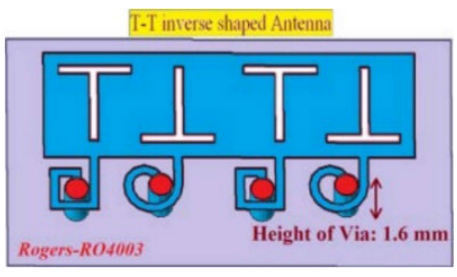

(b)

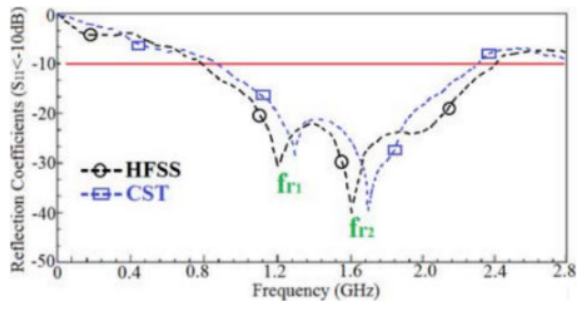

(c)

FIGURE 25. Antenna, and its reflection coefficient responses [41].

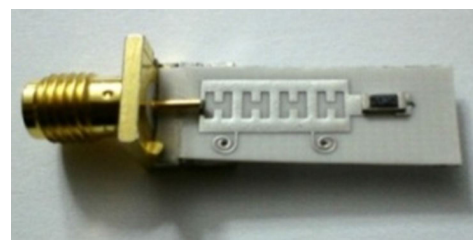

(a)

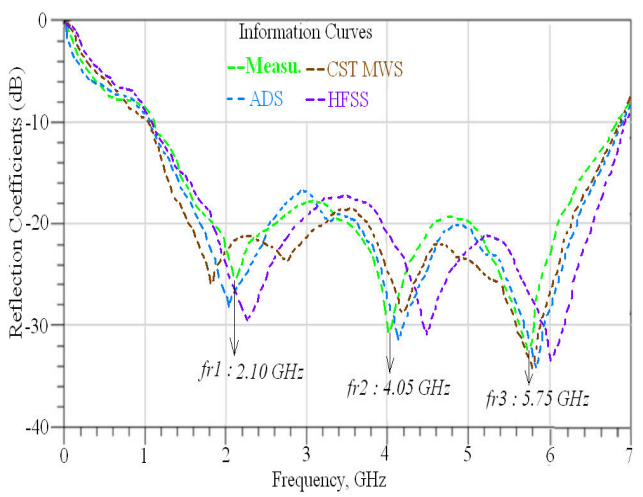

(c)

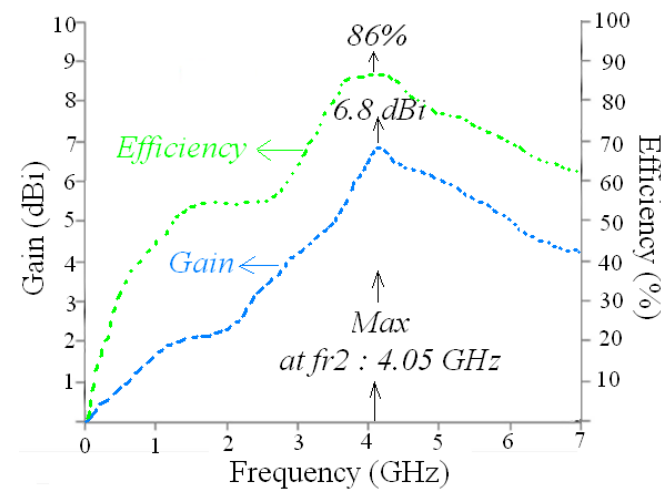

(e)

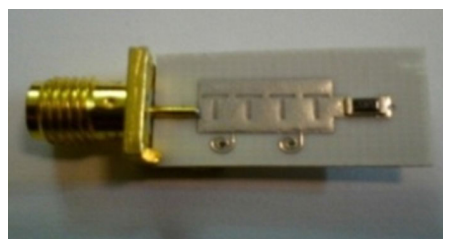

(b)

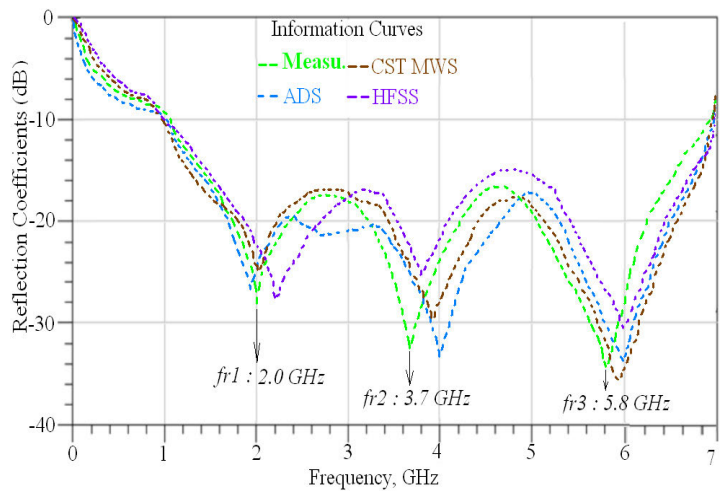

(d)

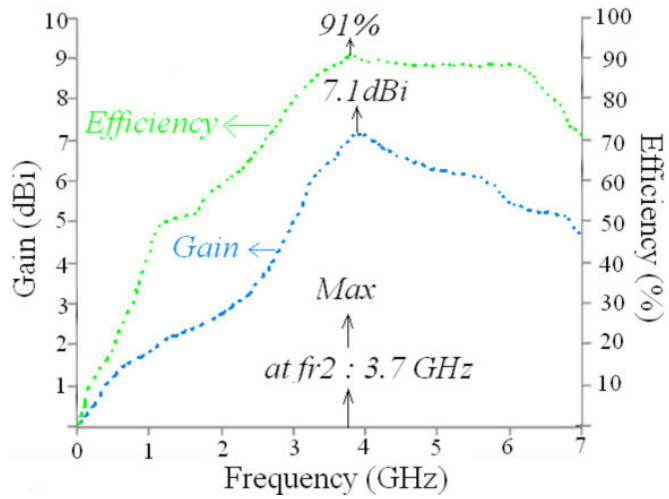

(f)

FIGURE 26. (a) H-antenna, (b) T-antenna, (c) H-antenna's reflection-coefficient responses, (d) T-antenna's reflection-coefficient responses, (e) H-antenna's gain and radiation efficiency responses, and (f) T-antenna's gain and radiation efficiency response[42].

bandwidth, the gain of the $\mathrm{H}$-formed unit-cell antenna ranges from $2 \mathrm{dBi}$ to $6.8 \mathrm{dBi}$ and its radiation efficiency ranges from $50 \%$ to $86 \%$. Over its bandwidth, the gain of the T-shaped unit-cell antenna ranges from $2 \mathrm{dBi}$ to $7.1 \mathrm{dBi}$ and its radiation efficiency ranges from $48 \%$ to $91 \%$. The specifications of the proposed antennas make them potential candidates for integration into wireless communication systems and portable microwave devices such as transceivers.

In [43], MTM CRLH-TLs are adopted for the implementation of a new miniaturized broadband antenna. The antenna is tuned for improved frequency bandwidth, efficiency and radiation pattern and it offers an enhanced gain performance. 


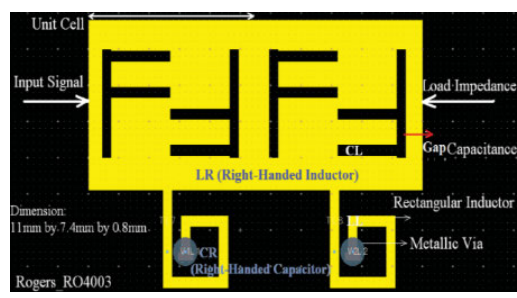

(a)

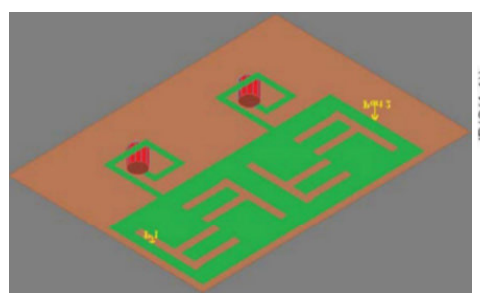

(b)

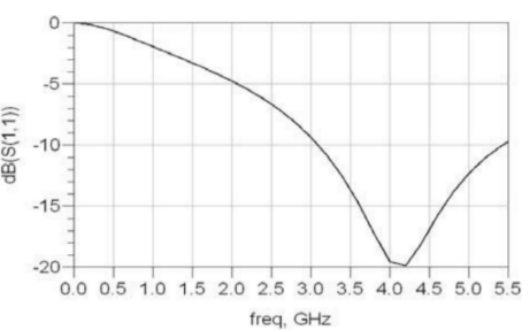

(c)

FIGURE 27. CRLH UWB antenna and its reflection coefficient response [43].

As shown in Fig.27, an inductively grounded (through a metal via-hole) rectangular radiation patch with two inverted F-formed slits printed on it is used for the realization of the CRLH-TL. For the implementation of the antenna, two CRLH-TL unit-cells are adopted to have a bandwidth coverage from $3.1-5.4 \mathrm{GHz}$, corresponding to $4.11 \%$ practical bandwidth. The antenna's total physical dimension is $11 \times 7.4 \times 0.8 \mathrm{~mm}^{3}$ or $0.15 \lambda_{0} \times 0.1 \lambda_{0} \times 0.011 \lambda_{0}$, where $\lambda_{0}$ is the wavelength of the free space at a $4.25 \mathrm{GHz}$ operational frequency. Its optimum gain and radiation efficiency have occurred at $5.4 \mathrm{GHz}$, which are $6.4 \mathrm{dBi}$ and $89.04 \%$. Some of the applications in various wireless technologies include (but are not limited) WLAN, WiMAX and WiFi.

In [44], a MTM antenna applying the principle of CRLH-TL is presented. Fig.28 shows that the radiation cells configurations are based on L- and F- formed slots which are etched on the radiation patches for organizing a series-capacitance impact $\left(C_{L}\right)$. Also, the radiation cells consist the spirals and via-holes for the shunt-inductances realization $\left(L_{L}\right)$. By cascading the suitable number of cells, the desirable antennas for VHF and UHF bands are modelled. First-antenna with four L-formed cells is implemented on the Rogers RO4003 layer with thickness of $0.8 \mathrm{~mm}$ and $\varepsilon_{r}=$ 3.38 so that each of cells takes an area of $2.3 \times 4.9 \mathrm{~mm}^{2}$. The antenna supports the frequency bandwidth of $0.2-1.8 \mathrm{GHz}$, which relates to $160 \%$ feasible-bandwidth. The antenna resonates at the operating frequencies of $600,850,1200$, and $1550 \mathrm{MHz}$ with the highest gain $(3.4 \mathrm{dBi})$ and efficiency $(88 \%)$ at $1550 \mathrm{MHz}$. To improve the antenna performances, a second antenna is modeled by adding an extra cell to the first antenna, changing the slot layout to an F-formed and enhancing the thickness of the layer to $1.6 \mathrm{~mm}$. F-formed antenna with a dimension of $14.5 \times 4.4 \times 1.6 \mathrm{~mm}^{3}$ covers a the requency band of $0.11-2.10 \mathrm{GHz}$ with five resonance frequencies at 450, 725, 1150, 1670, and $1900 \mathrm{MHz}$ relating to $180.1 \%$ feasible-bandwidth. The antenna's highest gain and efficiency occurred at $1900 \mathrm{MHz}$ are $4.5 \mathrm{dBi}$ and $95 \%$.

\section{SIMPLIFIED COMPOSITE RIGHT/LEFT-HANDED TRANSMISSION LINE (SCRLH-TL)}

The emergence of the simplified CRLH-TL or SCRLH-TL and its applications in antenna designs has caused in antennas with broad bandwidth, proper radiation patterns, and small structures that can be easily manufactured applying traditional techniques [47], [80], [81]. Unlike CRLH-TL, the SCRLH-TL resonates at zeroth and positive modes rather than negative order modes due to the absence of $\mathrm{LH}$ capacitance or inductance. When radiating at positive modes, the antenna achieves a higher efficiency in comparison with when it is radiating at the zeroth and negative modes. This advantage makes SCRLH-TL a proper nomine for modelling UWB antennas with a compact physical footprint area.

In [81], the feasible study of a new planar antenna model is investigated. The antenna is synthesized utilizing SCRLHTL. The SCRLH-TL is a version of a traditional CRLH-TL without the presence of a shunt-inductor in the unit-cell. Exhibiting a $\mathrm{RH}$ response having nonlinear dispersion characteristics and a smooth Bloch-impedance distribution, the SCRLH-TL attracts a lot of interest. As shown in Fig.29, three small rectangular patch radiators are placed in the region of the inner slot of the antenna. In Fig.29, each patch radiator has an E-shaped notch and a larger E-shaped notch is near the antenna's $50 \Omega$ terminal. The SCRLHTL property is inherent in the E-formed notches. The antenna's impedance bandwidth is determined by the gap (on the slot) between the smaller patches and the conductor (next to the larger E-formed notch). The radiation characteristics of the antenna are dependent on the sizes of the smaller patches. A conductor-backed CPW TL is used to fee the antenna. The antenna can operate within frequency band of $0.7-8 \mathrm{GHz}$, which relates to a bandwidth of $7.3 \mathrm{GHz}$ and a fractional bandwidth of $167.81 \%$. Over this bandwidth, the antenna has resonances at 4.75 and $7 \mathrm{GHz}$. The gain and radiation efficiency at $4.75 \mathrm{GHz}$ are $4 \mathrm{dBi}$ and $80 \%$. The same parameters at $7 \mathrm{GHz}$ are $3.6 \mathrm{dBi}$ and $73 \%$. The measured performances of the antenna were used for its validation. The antenna's size is $0.0504 \lambda_{0} \times 0.0462 \lambda_{0} \times 0.0018 \lambda_{0}$ in terms of the wavelength of the free-space at $700 \mathrm{MHz}$.

Feasible study of an innovative planar array antenna based on a SCRLH-TL for implement in circularly polarized (CP) synthetic aperture radar (SAR) systems operated in UHF, L, S and C-Bands is presented in [48]. Fig.30 shows that the array antenna includes of $6 \times 6$ matrix of spiral formed radiating elements that are stimulated by proximity-coupled, single feed-line. Pattern synthesis approach is applied to specify the excitation coefficients (amplitude and phase) to 

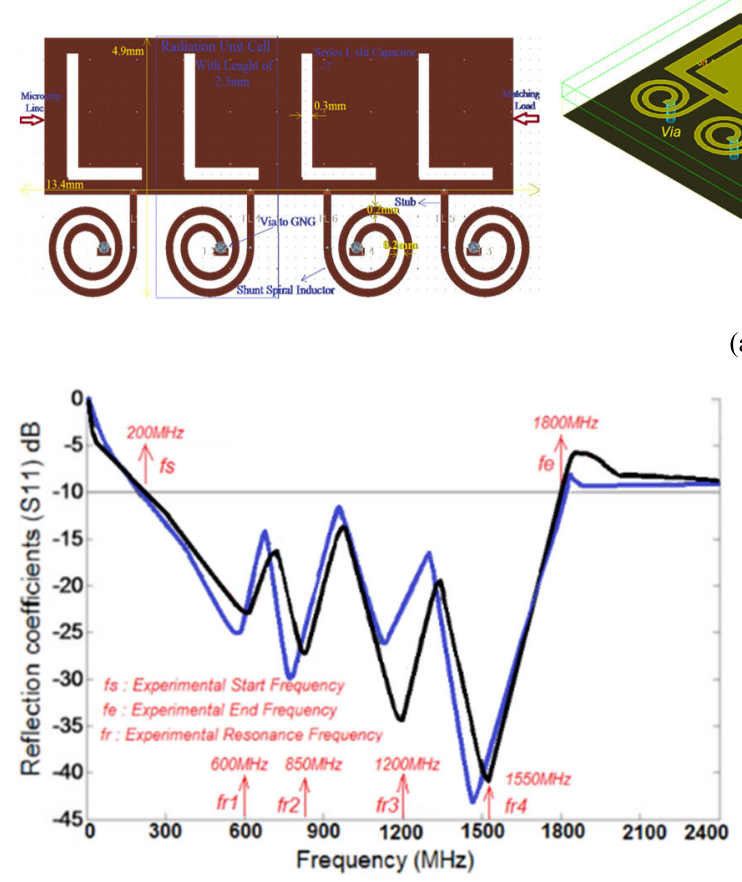

(b)

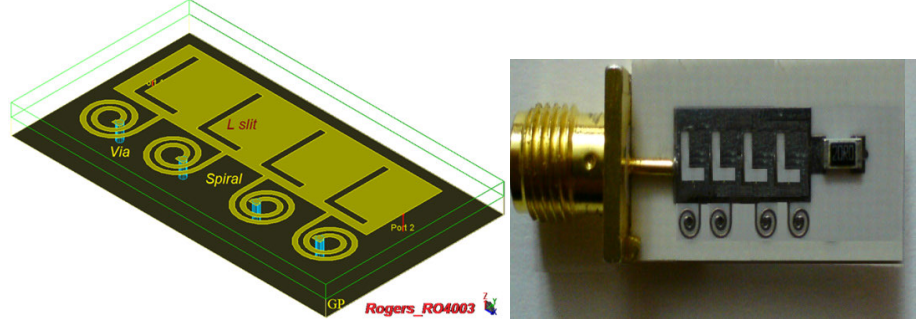

(a)

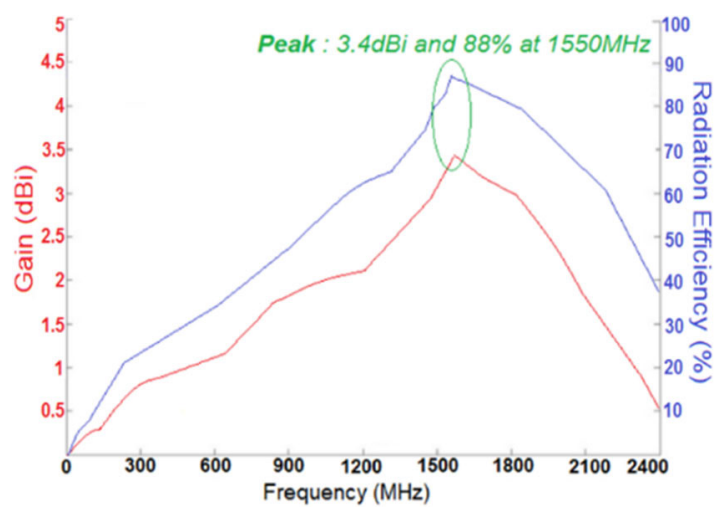

(c)

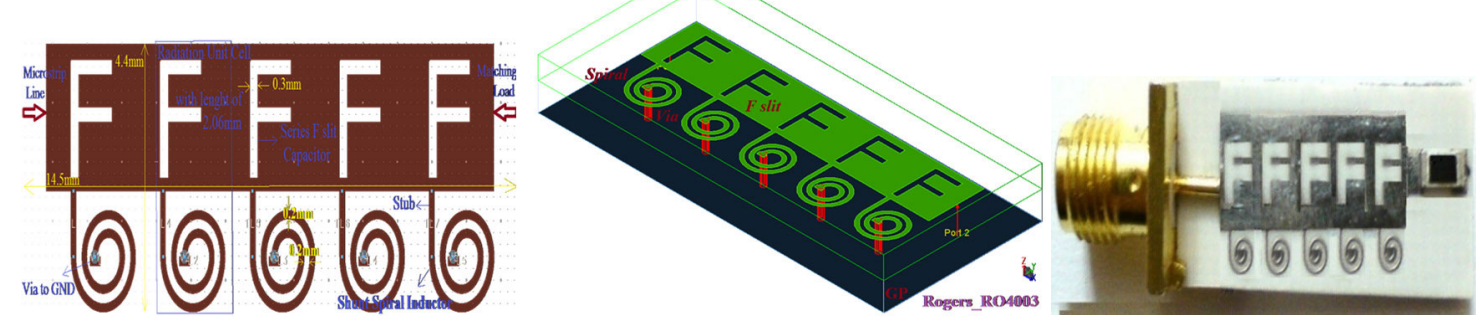

(d)

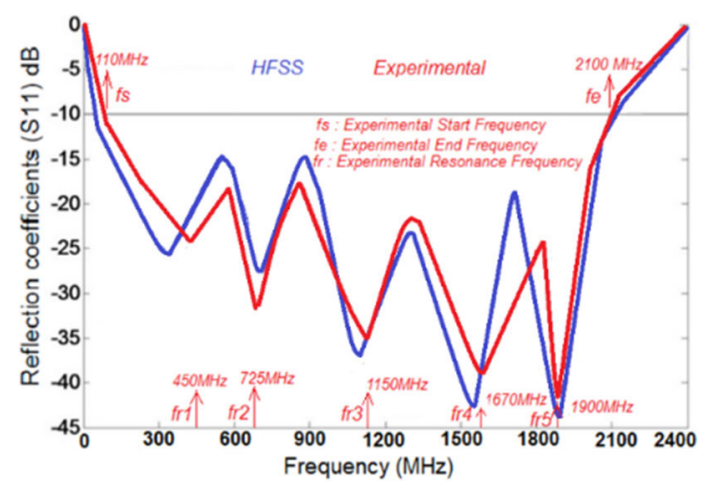

(e)

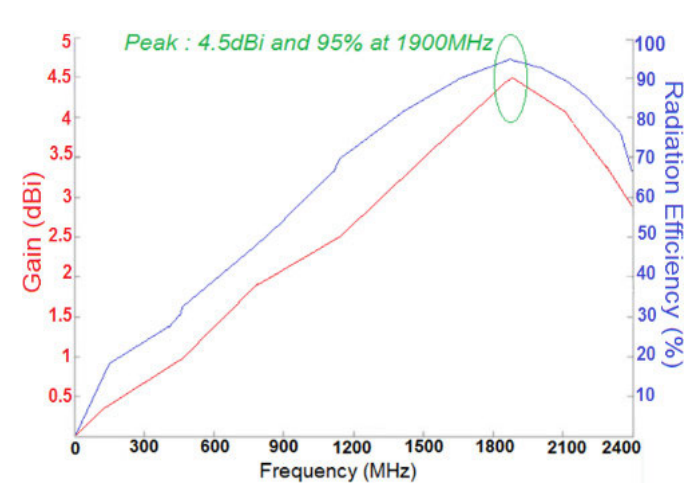

(f)

FIGURE 28. (a) L-shaped antenna, (b) its reflection coefficient responces, (c) its radiation gain and efficiency, (d) F-shaped antenna, (e) its reflection coefficients responces, and (f) its radiation gain and efficiency [44].

apply to the individual array elements to obtain the essential pattern form. The array antenna's size is $111.5 \times 96.06 \mathrm{~mm}^{2}$. Its frequency bandwidth is $3.85 \mathrm{GHz}$ covering the band of $0.3-4.15 \mathrm{GHz}$, relating to a practical bandwidth of $173 \%$. Highest gain and radiation efficiency are $4.8 \mathrm{dBi}$ and $79.5 \%$, which have been happened at $2.40 \mathrm{GHz}$. The antenna's $3 \mathrm{~dB}$ axial ratio bandwidth is $3.94 \mathrm{GHz}$ from $0.144-4.66 \mathrm{GHz}$. The beamwidth of the antenna in the azimuth and elevation planes change between $60^{\circ}$ and $120^{\circ}$ across its operational frequency range. The antenna design satisfies the challenging 


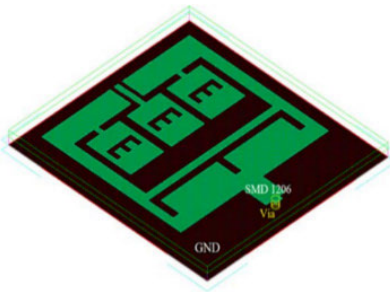

(a)

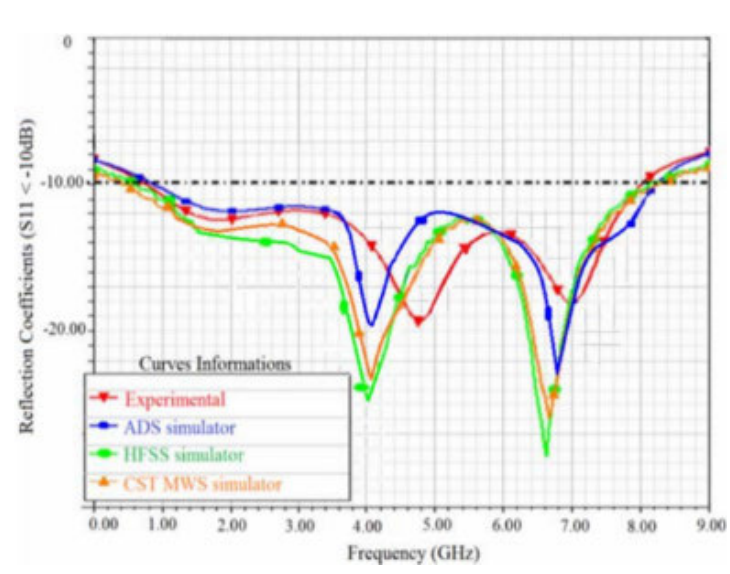

(e)

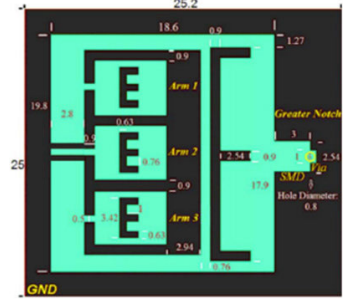

(b)

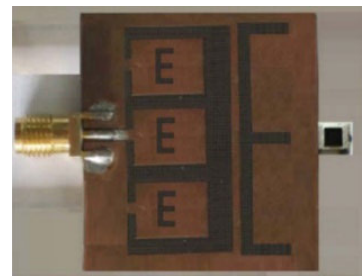

(c)

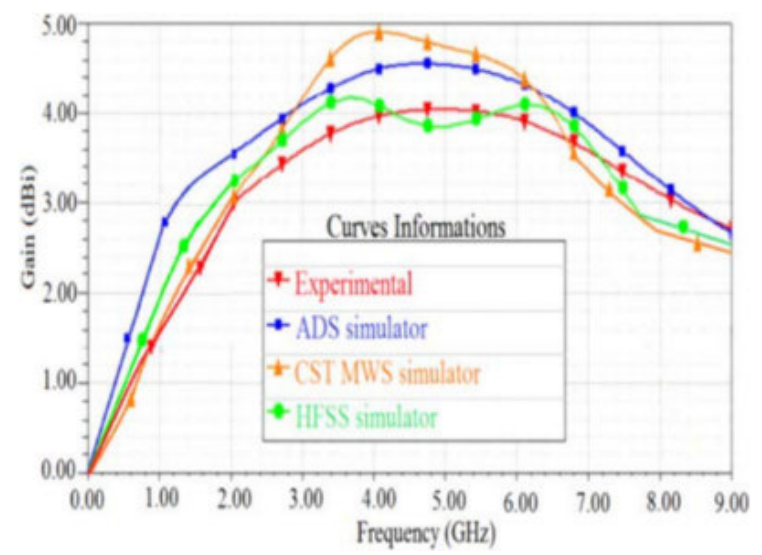

(f)

FIGURE 29. Test antenna prototype, its reflection coefficient and gain responses [81].

electrical and physical specifications demanded for CP-SAR employed on-board unmanned aerial vehicles (UAVs).

The innovative SCRLH-TL antennas for UWB applications have illustrated in [49] and depicted in Fig.31, which display excellent radiation properties. The SCRLH-TLs are realized applying F-formed and $\mathrm{T}$-formed slots on the GND and radiating arms. The impedance matching of the coplanar waveguide feed-line to the antenna was increased by integrating an $\mathrm{H}$-formed microstrip stub into the feedline. The antenna works inside $0.65-9.2 \mathrm{GHz}$, that relates to an feasible bandwidth of $173.6 \%$. The highest gain and efficiency are $3.5 \mathrm{dBi}$ and $70 \%$ at $4.5 \mathrm{GHz}$. Unlike conventional antennas, the antenna's size is not compromised with the introduction of extra patches utilizing the proposed method. The antenna dimensions are $25 \mathrm{~mm} \times 15 \mathrm{~mm}$ $\times 1.6 \mathrm{~mm}$ or $0.054 \lambda_{0} \times 0.032 \lambda_{0} \times 0.003 \lambda_{0}$, where $\lambda_{0}$ is the free-space wavelength at $0.65 \mathrm{GHz}$.

\section{TWO- AND THREE- DIMENSIONAL TRANSMISSION LINE METAMATERIALS}

It is good to note that 1D MTM-TLs are the CRLH structures considered so far. However, 2D and 3D MTM-TLs can also be realized. Instead of providing full details on the available realizations of 2D and 3D MTM-TLs, this section mainly focuses on the generic ideas for the synthesis of 2D and 3D MTM-TLs to showcase the relevant works which are applicable to this topic. In [50], the implementation of 2D MTM-TLs involving the introduction of a square grid of
TLs having capacitors in series and inductors in parallel at the nodes is presented. A similar implementation without the use of lumped elements has been proposed in [82]. The proposed structure in [82] is a mushroom model that can be depicted and analysed by a 2D CRLH-TL. Its dispersion curve indicates both forward wave propagation and backward wave propagation properties. Further realizations of $3 \mathrm{D}$ MTM-TLs can be found in [83] and [84]. In [83] and [84], implementation of super-lens and the experimental validation of the augmentation of evanescent waves are carried out, respectively. Other notable works include [85] and [86]. In [85] and [86], the rotated TL matrix approach and the symmetrical condensed node of TL matrix are adopted, respectively, as structures stemming from the traditional dielectrics and their 3D TL models [87]. It is good to note that other methods for the realization of 3D MTM-TLs based on split rings (which are arranged in cubic lattices) have been proposed [88], [89]. However, fishnet structures constructed by means of microfabrication or nanofabrication technologies appear to be the most promising realizations of bulk MTM-TLs. More specifically, at quasi-optical or optical frequencies, [90], [91].

\section{PERFORMANCE PARAMETERS OF MTM TL-BASED ANTENNAS IN COMPARISON WITH THEIR CONVENTIONAL ONES}

In this section the effects of the metamaterial concept on the antenna's performance parameters in terms of dimensions, 


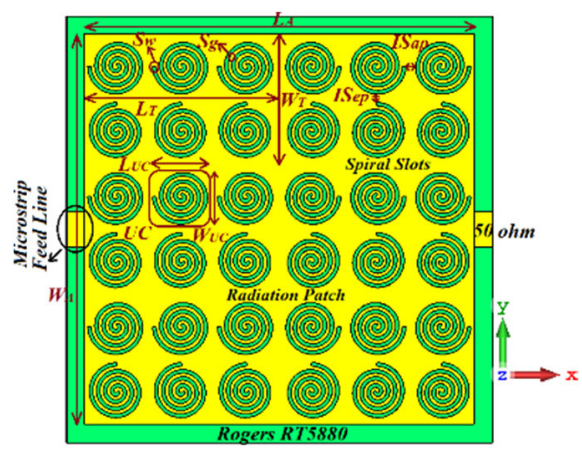

(a)

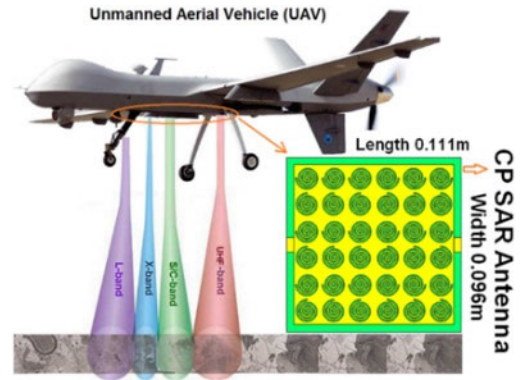

(b)

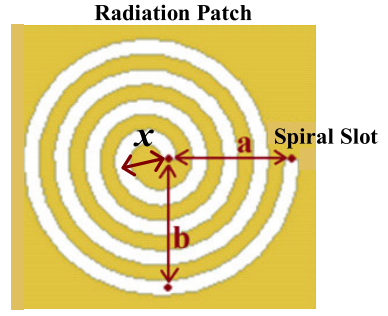

(c)

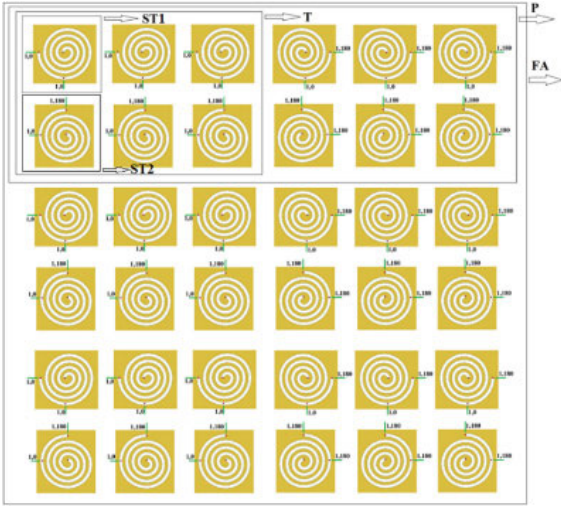

(d)

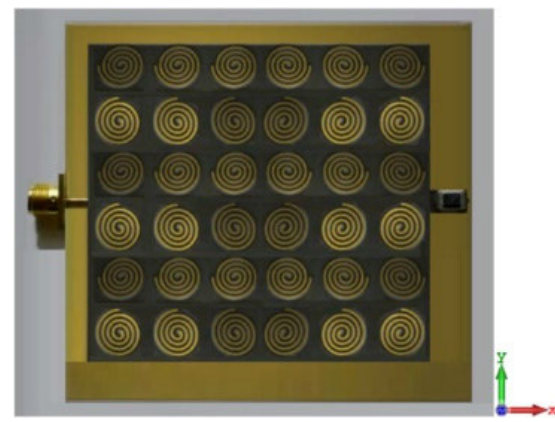

(e)

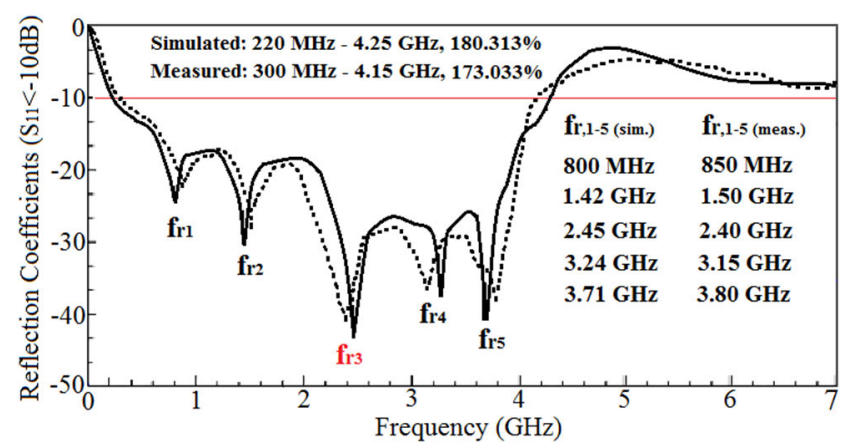

(f)

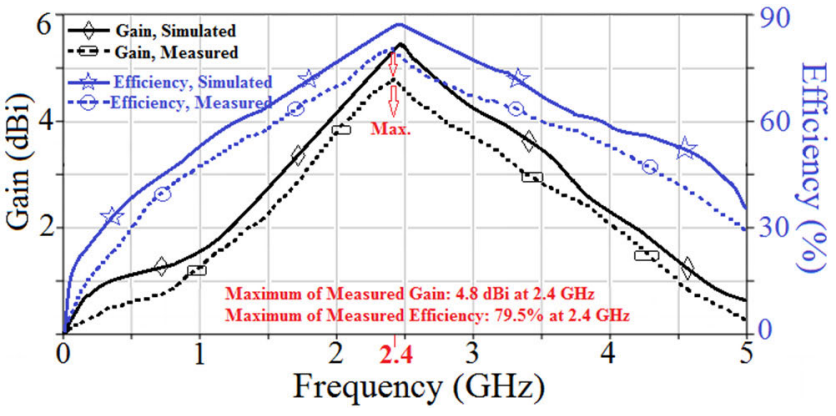

(g)

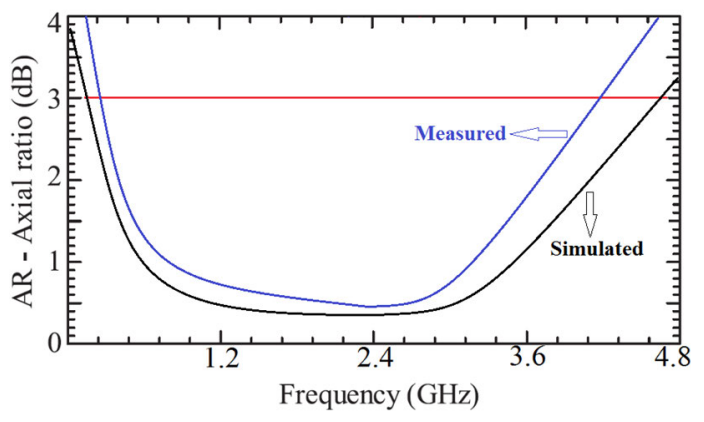

(h)

FIGURE 30. (a) Array antenna with direct feed-line, (b) Array antenna onboard a UAV, (c) radiating unit-cell element, (d) applied cross-polarization suppression methods. (e) Photograph of the SAR array antenna, (f) reflection coefficient responses, (g) Gain and radiation efficiency, and (h) Axial-ratio at $\theta=0^{\circ}[48]$.

impedance bandwidth, radiation gain, radiation efficiency, simplicity, and cost effective have been reviewed and compared with their conventional ones without applying the metamaterial principle. It is shown that, the metamaterial 


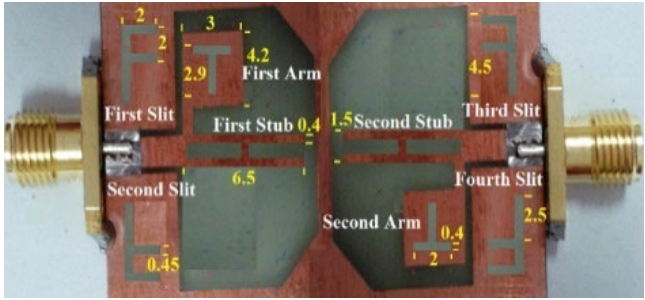

(a)

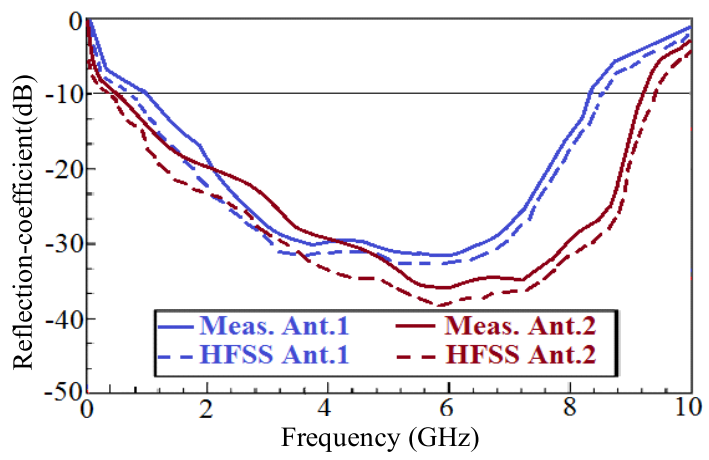

(c)

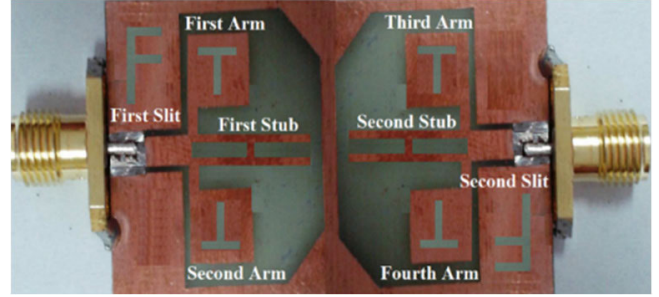

(b)

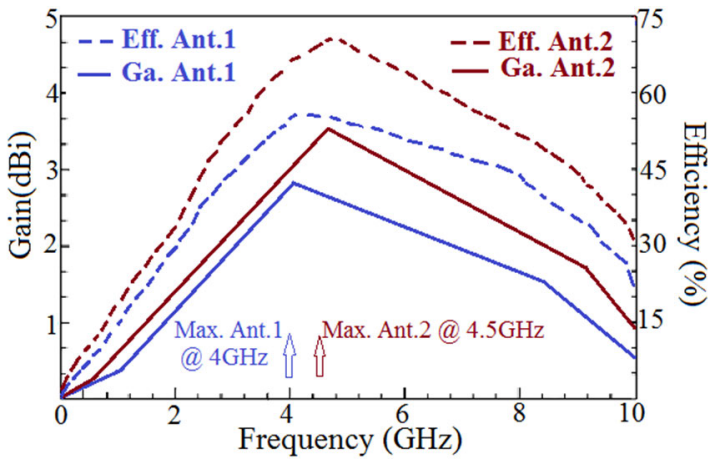

(d)

FIGURE 31. (a) Antenna \# 1, (b) Antenna \#2, (c) reflection-coefficient responses, and (d) measured gain and radiation efficiency responses [49].

TABLE 1. Performance parameters of the MTM TL-based antennas in comparison with their conventional structures.

\begin{tabular}{|c|c|c|c|c|c|}
\hline References & Dimensions $\left(\mathrm{mm}^{3}\right)$ & Bandwidth & Gain & Efficiency & $\begin{array}{l}\text { Complexity after apply } \\
\text { MTM concept }\end{array}$ \\
\hline [31]-Conventional & $18.7 \times 6 \times 1.6$ & $56.2-64.6 \mathrm{GHz}$ & $5.8 \mathrm{dBi}$ & $63.5 \%$ & - \\
\hline [31]-with MTM & $18.7 \times 6 \times 1.6$ & $55-65 \mathrm{GHz}$ & $7.8 \mathrm{dBi}$ & $84.2 \%$ & Complexity not increased \\
\hline [34]-Conventional & $17.5 \times 32.15 \times 1.6$ & $2-6.5 \mathrm{GHz}$ & $1.2 \mathrm{dBi}$ & $20 \%$ & - \\
\hline [34]-with MTM & $17.5 \times 32.15 \times 1.6$ & $0.85-7.90 \mathrm{GHz}$ & $5.12 \mathrm{dBi}$ & $\sim 80 \%$ & Complexity not increased \\
\hline [36]-Conventional & $25 \times 10 \times 1.6$ & $1-4.2 \mathrm{GHz}$ & $1.5 \mathrm{dBi}$ & $35 \%$ & - \\
\hline [36]-with MTM & $25 \times 10 \times 1.6$ & $0.75-4.5 \mathrm{GHz}$ & $3.5 \mathrm{dBi}$ & $60 \%$ & Complexity not increased \\
\hline [37]-Conventional & $27 \times 35 \times 1.6$ & $0.4-2.3 \mathrm{GHz}$ & $2.21 \mathrm{dBi}$ & $51.4 \%$ & - \\
\hline [37]-with MTM & $40 \times 35 \times 1.6$ & $0.41-4.1 \mathrm{GHz}$ & $4.45 \mathrm{dBi}$ & $85.8 \%$ & Complexity not increased \\
\hline [49]-Conventional & $25 \times 15 \times 1.6$ & $1-8.4 \mathrm{GHz}$ & $2.8 \mathrm{dBi}$ & $55 \%$ & - \\
\hline [49]-with MTM & $25 \times 15 \times 1.6$ & $0.65-9.2 \mathrm{GHz}$ & $3.5 \mathrm{dBi}$ & $70 \%$ & Complexity not increased \\
\hline [51]-Conventional (1) & $30 \times 22 \times 0.8$ & $4.0-5.50 \mathrm{GHz}$ & $3.6 \mathrm{dBi}$ & $78.5 \%$ & - \\
\hline [51]-with MTM (1) & $30 \times 22 \times 0.8$ & $2.9-6.41 \mathrm{GHz}$ & $4 \mathrm{dBi}$ & $81.2 \%$ & Complexity not increased \\
\hline [51]-Conventional (2) & $30 \times 22 \times 0.8$ & $3.82-5.80 \mathrm{GHz}$ & $3.9 \mathrm{dBi}$ & $80.2 \%$ & - \\
\hline [51]-with MTM (2) & $30 \times 22 \times 0.8$ & $2.6-6.6 \mathrm{GHz}$ & $4.4 \mathrm{dBi}$ & $82.6 \%$ & Complexity not increased \\
\hline [53]-Conventional & $48.32 \times 43.72 \times 0.8$ & $1.15-1.8 \mathrm{GHz}$ & $0.35 \mathrm{dBi}$ & $22.8 \%$ & - \\
\hline [53]-with MTM & $48.32 \times 43.72 \times 0.8$ & $0.115-2.90 \mathrm{GHz}$ & $2.35 \mathrm{dBi}$ & $78.8 \%$ & Complexity not increased \\
\hline [55]-Conventional & $21.2 \times 15 \times 0.8$ & $2.2-4.5 \mathrm{GHz}$ & $2.75 \mathrm{dBi}$ & $47.15 \%$ & - \\
\hline [55]-with MTM & $21.2 \times 15 \times 0.8$ & $0.800-6.05 \mathrm{GHz}$ & $5.35 \mathrm{dBi}$ & $84.12 \%$ & Complexity not increased \\
\hline [81]-Conventional & $21.6 \times 19.8 \times 0.8$ & $2.5-6.2 \mathrm{GHz}$ & $3.6 \mathrm{dBi}$ & $60 \%$ & - \\
\hline [81]-with MTM & $21.6 \times 19.8 \times 0.8$ & $0.7-8 \mathrm{GHz}$ & $4 \mathrm{dBi}$ & $80 \%$ & Complexity not increased \\
\hline
\end{tabular}

transmission-based antennas cover wider frequency band with higher radiation characteristics and constant physical dimensions in comparison with their conventional ones. Additionally, after apply the metamaterial concept the complexity of the antennas has not increased, which makes them cost effective for mass production.

\section{CONCLUSION}

This review study has investigated CRLH MTM-TLs by considering their use in the design of antennas presented in available literature. To analyse the critical features of metamaterial theory and concept, several examples are used. Comparisons on the basis of physical size, frequency bandwidth, materials, gain, radiation efficiency, and radiation patterns are made for all the examples that are based on CRLH MTM-TLs. This review study has illustrated that by integrating slots, interdigital capacitors, spiral- and meander lines-shaped stubs, and via-holes into the design of antennas, very broadband of frequency with high performances can be obtained. Additionally, the use of metamaterial allows for structural simplicity. In addition, MTM antennas display better performances with a cost effective fabrication process. 
As revealed in all the metamaterial-based antenna design examples, foot-print area decrement is an serious topic of study that has a straightforward influence for the growth of the next generation wireless communication systems. Thus, a broad range of design possibilities is introduced in this study to highlight the improvement of the performance parameters that is rare in available literature. Therefore, this review work provides a wide overview of the early-stage concepts of metamaterial-based design as a thorough reference for specialist antenna designers.

\section{REFERENCES}

[1] M. D. Sindreu, J. Naqui, J. Selga, P. Vélez, J. Bonache, and F. Martín, "Composite right-/left-handed transmission line metamaterials," in Wiley Encyclopedia of Electrical and Electronics Engineering. Hoboken, NJ, USA: Wiley, Apr. 2013, pp. 1-25, doi: 10.1002/047134608X.W8195.

[2] A. Lai, T. Itoh, and C. Caloz, "Composite right/left-handed transmission line metamaterials," IEEE Microw. Mag., vol. 5, no. 3, pp. 34-50, Sep. 2004

[3] D. Kennedy, "Breakthrough of the year: The runners-up," Science, vol. 302, no. 5653, pp. 2039-2045, 2003.

[4] C.-J. Lee, W. Huang, A. Gummalla, and M. Achour, "Small antennas based on CRLH structures: Concept, design, and applications," IEEE Antennas Propag. Mag., vol. 53, no. 2, pp. 10-25, Apr. 2011

[5] F. Martin, J. Bonache, M. Durán-Sindreu, J. Naqui, F. Paredes, and G. Zamora, "Artificial transmission lines," in Wiley Encyclopedia of Electrical and Electronics Engineering, J. Webster, Ed. New York, NY, USA: Wiley, 2012, pp1-25.

[6] Y. J. Cheng, W. Hong, and K. Wu, "Millimeter-wave half mode substrate integrated waveguide frequency scanning antenna with quadri-polarization," IEEE Trans. Antennas Propag., vol. 58, no. 6, pp. 1848-1855, Jun. 2010.

[7] D. Zelenchuk, A. J. Martinez-Ros, T. Zvolensky, J. L. Gomez-Tornero, G. Goussetis, N. Buchanan, D. Linton, and V. Fusco, "W-band planar wide-angle scanning antenna architecture," J. Infr., Millim., Terahertz Waves, vol. 34, no. 2, pp. 127-139, Jan. 2013.

[8] M. Poveda-Garcia, J. Oliva-Sanchez, R. Sanchez-Iborra, D. Canete-Rebenaque, and J. L. Gomez-Tornero, "Dynamic wireless power transfer for cost-effective wireless sensor networks using frequencyscanned beaming," IEEE Access, vol. 7, pp. 8081-8094, Jan. 2019.

[9] M. K. Emara, D. J. King, H. Nguyen, S. Abielmona, and S. Gupta, "Multi-port slot array antenna for millimeter-wave direction finding and beam-forming applications," in Proc. 13th Eur. Conf. Antennas Propag. (EuCAP), Krakow, Poland, Mar./Apr. 2019, pp. 1-3.

[10] M. Poveda-Garcia, A. Gomez-Alcaraz, D. Canete-Rebenaque, A. S. Martinez-Sala, and J. L. Gomez-Tornero, "RSSI-based directionof-departure estimation in Bluetooth low energy using an array of frequency-steered leaky-wave antennas," IEEE Access, vol. 8, pp. 9380-9394, Jan. 2020.

[11] C. Caloz and T. Itoh, Electromagnetic Metamaterials: Transmission Line Theory and Microwave Applications. New York, NY, USA: Wiley, 2006.

[12] M. Guglielmi and D. R. Jackson, "Broadside radiation from periodic leaky-wave antennas," IEEE Trans. Antennas Propag., vol. 41, no. 1, pp. 31-37, Jan. 1993.

[13] P. Burghignoli, G. Lovat, and D. R. Jackson, "Analysis and optimization of leaky-wave radiation at broadside from a class of 1-D periodic structures," IEEE Trans. Antennas Propag., vol. 54, no. 9, pp. 2593-2604, Sep. 2006.

[14] M. Garcia-Vigueras, J. L. Gomez-Tornero, G. Goussetis, A. R. Weily, and Y. J. Guo, "Enhancing frequency-scanning response of leakywave antennas using high-impedance surfaces," IEEE Antennas Wireless Propag. Lett., vol. 10, pp. 7-10, 2011.

[15] D. Xie and L. Zhu, "Microstrip leaky-wave antennas with nonuniform periodical loading of shorting pins for enhanced frequency sensitivity," IEEE Trans. Antennas Propag., vol. 66, no. 7, pp. 3337-3345, Jul. 2018.

[16] R. Guzman-Quiros, J. L. Gomez-Tornero, A. R. Weily, and Y. J. Guo, "Electronic full-space scanning with 1-D Fabry-Pérot LWA using electromagnetic band-gap," IEEE Antennas Wireless Propag. Lett., vol. 11, pp. 1426-1429, Dec. 2012.
[17] Z. Li, Y. J. Guo, S.-L. Chen, and J. Wang, "A period-reconfigurable leaky-wave antenna with fixed-frequency and wide-angle beam scanning," IEEE Trans. Antennas Propag., vol. 67, no. 6, pp. 3720-3732, Jun. 2019.

[18] N. Nguyen-Trong, L. Zou, C. Fumeaux, and C. Caloz, "Ultra-high and tunable sensitivity leaky-wave scanning using gain-loss C-section phasers," App. Phys., 2017. [Online]. Available: https://arxiv.org/abs/1706.06222

[19] D. K. Karmokar, Y. J. Guo, S.-L. Chen, and T. S. Bird, "Composite right/left-handed leaky-wave antennas for wide-angle beam scanning with flexibly chosen frequency range," IEEE Trans. Antennas Propag., vol. 68, no. 1, pp. 100-110, Jan. 2020.

[20] F. Martin, J. Bonache, F. A. Falcone, M. Sorolla, and R. Marqués, "Split ring resonator-based left-handed coplanar waveguide," Appl. Phys. Lett., vol. 83, no. 22, pp. 4652-4654, 2003.

[21] C. R. Simovski, P. A. Belov, and S. He, "Backward wave region and negative material parameters of a structure formed by lattices of wires and split-ring resonators," IEEE Trans. Antennas Propag., vol. 51, no. 10, pp. 2582-2591, Oct. 2003.

[22] F. Falcone, T. Lopetegi, M. A. Laso, J. D. Baena, J. Bonache, M. Beruete, R. Marqués, F. Martín, and M. Sorolla, "Principle applied to the design of metasurfaces and metamaterials," Phys. Rev. Lett., vol. 93, no. 19, 2004, Art. no. 197401.

[23] J. D. Baena, J. Bonache, F. Martin, R. M. Sillero, F. Falcone, T. Lopetegi, M. A. G. Laso, J. Garcia-Garcia, I. Gil, M. F. Portillo, and M. Sorolla, "Equivalent-circuit models for split-ring resonators and complementary split-ring resonators coupled to planar transmission lines," IEEE Trans. Microw. Theory Techn., vol. 53, no. 4, pp. 1451-1461, Apr. 2005.

[24] M. Gil, J. Bonache, I. Gil, J. García-García, and F. Martín, "On the transmission properties of left-handed microstrip lines implemented by complementary split rings resonators," Int. J. Num. Model., Electron. Netw., Devices Fields, vol. 19, no. 2, pp. 87-103, 2006.

[25] F. Falcone, T. Lopetegi, J. D. Baena, R. Marques, F. Martin, and M. Sorolla, "Effective negative- $\epsilon$ stop-band microstrip lines based on complementary split ring resonators," IEEE Microw. Wireless Compon. Lett., vol. 14, no. 6, pp. 280-282, Jun. 2004, doi: 10.1109/LMWC.2004.828029.

[26] A. Velez, F. Aznar, J. Bonache, M. C. Velazquez-Ahumada, J. Martel, and F. Martin, "Open complementary split ring resonators (OCSRRs) and their application to wideband CPW band pass filters," IEEE Microw. Wireless Compon. Lett., vol. 19, no. 4, pp. 197-199, Apr. 2009.

[27] M. Duran-Sindreu, A. Velez, F. Aznar, G. Siso, J. Bonache, and F. Martin, "Applications of open split ring resonators and open complementary split ring resonators to the synthesis of artificial transmission lines and microwave passive components," IEEE Trans. Microw. Theory Techn., vol. 57, no. 12, pp. 3395-3403, Dec. 2009.

[28] J. Martel, R. Marques, F. Falcone, J. D. Baena, F. Medina, F. Martin, and M. Sorolla, "A new LC series element for compact band pass filter design," IEEE Microw. Wireless Compon. Lett., vol. 14, no. 5, pp. 210-212, May 2004.

[29] R. W. Ziolkowski, P. Jin, and C.-C. Lin, "Metamaterial-inspired engineering of antennas," Proc. IEEE, vol. 99, no. 10, pp. 1720-1731, Oct. 2011.

[30] Y. Dong and T. Itoh, "Metamaterial-based antennas," Proc. IEEE, vol. 100, no. 7, pp. 2271-2285, Jul. 2012.

[31] M. Alibakhshikenari, B. S. Virdee, M. Khalily, P. Shukla, C. H. See, R. A. Abd-Alhameed, F. Falcone, and E. Limiti, "Beam-scanning leakywave antenna based on CRLH-metamaterial for millimeter-wave applications," IET Microw., Antennas Propag., vol. 13, no. 8, pp. 1129-1133, Jul. 2019.

[32] M. Alibakhshi-Kenari, A. Andújar, and J. Anguera, "New compact printed leaky-wave antenna with beam steering," Microw. Opt. Technol. Lett., vol. 58, no. 1, pp. 215-217, Jan. 2016.

[33] A. Sanada, C. Caloz, and T. Itoh, "Novel zeroth-order resonance in composite right/left-handed transmission line resonators," in Proc. Asia Pacific Microw. Conf., Seoul, South Korea, 2003, pp. 1588-1592.

[34] M. Alibakhshikenari, B. S. Virdee, A. Ali, and E. Limiti, "Extended aperture miniature antenna based on CRLH metamaterials for wireless communication systems operating over UHF to C-band," Radio Sci., vol. 53, no. 2, pp. 154-165, Feb. 2018.

[35] M. Alibakhshikenari, B. S. Virdee, A. Ali, and E. Limiti, "Miniaturised planar-patch antenna based on metamaterial L-shaped unit-cells for broadband portable microwave devices and multiband wireless communication systems," IET Microw., Antennas Propag., vol. 12, no. 7, pp. 1080-1086, Jun. 2018. 
[36] M. Alibakhshi-Kenari, M. Naser-Moghadasi, R. A. Sadeghzadeh, B. S. Virdee, and E. Limiti, "New CRLH-based planar slotted antennas with helical inductors for wireless communication systems, RF-circuits and microwave devices at UHF-SHF bands," Wireless Pers. Commun., vol. 92, no. 3, pp. 1029-1038, Feb. 2017.

[37] M. Alibakhshi-Kenari, M. Naser-Moghadasi, R. A. Sadeghzadeh, B. S. Virdee, and E. Limiti, "Periodic array of complementary artificial magnetic conductor metamaterials-based multiband antennas for broadband wireless transceivers," IET Microw., Antennas Propag., vol. 10, no. 15, pp. 1682-1691, Dec. 2016.

[38] M. Alibakhshi-Kenari and M. Naser-Moghadasi, "Novel UWB miniaturized integrated antenna based on CRLH metamaterial transmission lines," AEU-Int. J. Electron. Commun., vol. 69, no. 8, pp. 1143-1149, Aug. 2015.

[39] M. Alibakhshi-Kenari, M. Naser-Moghadasi, R. A. Sadeghzadeh, B. S. Virdee, and E. Limiti, "Miniature CRLH-based ultra wideband antenna with gain enhancement for wireless communication applications," ICT Exp., vol. 2, no. 2, pp. 75-79, Jun. 2016.

[40] M. Alibakhshi-Kenari, M. Naser-Moghadasi, B. S. Virdee, A. Andújar, and J. Anguera, "Compact antenna based on a composite right/lefthanded transmission line," Microw. Opt. Technol. Lett., vol. 57, no. 8, pp. 1785-1788, Aug. 2015.

[41] M. Alibakhshikenari, B. S. Virdee, A. Andujar, J. Anguera, and E. Limiti, "CRLH metamaterial transmission line based-wideband planar antenna for operation across UHF/L/S-bands," in Proc. 11th Int. Congr. Engineered Mater. Platforms Novel Wave Phenomena (Metamaterials), Marseille, France, Aug. 2017, pp. 10-12.

[42] M. Alibakhshi-Kenari, M. Naser-Moghadasi, R. Ali Sadeghzadeh, and B. Singh Virdee, "Metamaterial-based antennas for integration in UWB transceivers and portable microwave handsets," Int. J. RF Microw. Comput.-Aided Eng., vol. 26, no. 1, pp. 88-96, Jan. 2016.

[43] M. Alibakhshi-Kenari, M. Naser-Moghadasi, R. A. Sadeghzadeh, B. S. Virdee, and E. Limiti, "Composite right/left-handed transmissionline based wideband antenna for WLAN, WiMAX and WiFi applications," in Proc. 8th Int. Symp. Telecommun. (IST), Sep. 2016, pp. 415-418.

[44] M. Alibakhshi-Kenari, R. A. Sadeghzadeh, and M. Naser-Moghadasi, "Composite right-left-handed-based antenna with wide applications in very-high frequency-ultra-high frequency bands for radio transceivers," IET Microw., Antennas Propag., vol. 9, no. 15, pp. 1713-1726, Dec. 2015.

[45] M. Alibakhshikenari, B. S. Virdee, and E. Limiti, "Compact single-layer traveling-wave antenna design using metamaterial transmission lines," Radio Sci., vol. 52, no. 12, pp. 1510-1521, Dec. 2017.

[46] M. Alibakhshi-Kenari, M. Naser-Moghadasi, R. A. Sadeghzadeh, B. S. Virdee, and E. Limiti, "Traveling-wave antenna based on metamaterial transmission line structure for use in multiple wireless communication applications," AEU-Int. J. Electron. Commun., vol. 70, no. 12, pp. 1645-1650, Dec. 2016

[47] X. Q. Lin, R. P. Liu, X. M. Yang, J. X. Chen, X. X. Yin, Q. Cheng, and T. J. Cui, "Arbitrarily dual-band components using simplified structures of conventional CRLH TLs," IEEE Trans. Microw. Theory Techn., vol. 54, no. 7, pp. 2902-2909, Jul. 2006.

[48] M. Alibakhshikenari, B. S. Virdee, and E. Limiti, "Wideband planar array antenna based on SCRLH-TL for airborne synthetic aperture radar application," J. Electromagn. Waves Appl., vol. 32, no. 12, pp. 1586-1599, Jun. 2018.

[49] R. A. Sadeghzadeh, M. Alibakhshi-Kenari, and M. Naser-Moghadasi, "UWB antenna based on SCRLH-TLs for portable wireless devices," Microw. Opt. Technol. Lett., vol. 58, no. 1, pp. 69-71, Jan. 2016.

[50] G. V. Eleftheriades, A. K. Iyer, and P. C. Kremer, "Planar negative refractive index media using periodically L-C Loaded transmission lines," IEEE Trans. Microw. Theory Techn., vol. 50, no. 12, pp. 2702-2712, Dec. 2002.

[51] M. Alibakhshi-Kenari, M. Naser-Moghadasi, and R. A. Sadeghzadeh, "Bandwidth and radiation specifications enhancement of monopole antennas loaded with split ring resonators," IET Microw., Antennas Propag., vol. 9, no. 14, pp. 1487-1496, Nov. 2015.

[52] M. Alibakhshikenari, B. S. Virdee, C. H. See, R. Abd-Alhameed, A. Ali, F. Falcone, and E. Limiti, "Wideband printed monopole antenna for application in wireless communication systems," IET Microw., Antennas Propag., vol. 12, no. 7, pp. 1222-1230, Jun. 2018.

[53] M. Alibakhshi-Kenari, M. Naser-Moghadasi, R. A. Sadeghzadeh, B. S. Virdee, and E. Limiti, "A new planar broadband antenna based on meandered line loops for portable wireless communication devices," Radio Sci., vol. 51, no. 7, pp. 1109-1117, Jul. 2016.
[54] M. Alibakhshi-Kenari, M. Naser-Moghadasi, R. A. Sadeghzadeh, B. S. Virdee, and E. Limiti, "Bandwidth extension of planar antennas using embedded slits for reliable multiband RF communications," AEU-Int. J. Electron. Commun., vol. 70, no. 7, pp. 910-919, Jul. 2016.

[55] M. A. Kenari, "Design and modeling of new UWB metamaterial planar cavity antennas with shrinking of the physical size for modern transceivers," Int. J. Antennas Propag., vol. 2013, pp. 1-12, Jul. 2013.

[56] C. A Balanis, Antenna Theory, Analysis and Design, 2nd ed. New York, NY, USA: Wiley, 1997.

[57] A. Oliner, Antenna Engineering Handbook, R. C. Johnson, Ed., 3rd ed. New York, NY, USA: McGraw-Hill, 1993.

[58] M. Mohammadi, F. H. Kashani, and J. Ghalibafan, "A partially ferritefilled rectangular waveguide with CRLH response and its application to a magnetically scannable antenna," J. Magn. Magn. Mater., vol. 491, Dec. 2019, Art. no. 165551

[59] M. Mohammadi, F. H. Kashani, and J. Ghalibafan, "Backfire-to-endfire scanning capability of a balanced metamaterial structure based on slotted ferrite-filled waveguid," Waves Random Complex Media, pp. 1-15, Aug. 2019, doi: 10.1080/17455030.2019.1654148.

[60] L. Liu, C. Caloz, and T. Itoh, "Dominant mode leaky-wave antenna with backfire-to-endfire scanning capability," Electron. Lett., vol. 38, no. 23, pp. 1414-1416, 2002.

[61] S. Lim, C. Caloz, and T. Itoh, "Metamaterial-based electronically controlled transmission-line structure as a novel leaky-wave antenna with tunable radiation angle and beamwidth," IEEE Trans. Microw. Theory Techn., vol. 53, no. 1, pp. 161-173, Jan. 2005.

[62] C. Damm, M. Maasc, R. Gonzalo, and R. Jakoby, "Tunable composite right/left-handed leaky wave antenna based on a rectangular waveguide using liquid crystals," in IEEE MTT-S Int. Microw. Symp. Dig., Anaheim, CA, USA, May 2010, pp. 13-16.

[63] S. Abielmona, H. V. Nguyen, and C. Caloz, "Analog direction of arrival estimation using an electronically-scanned CRLH leaky-wave antenna," IEEE Trans. Antennas Propag., vol. 59, no. 4, pp. 1408-1412, Apr. 2011.

[64] C. G. M. Ryan and G. V. Eleftheriades, "A dual-band leaky-wave antenna based on generalized negative-refractive-index transmission-lines," in Proc. IEEE Antennas Propag. Soc. Int. Symp., Jul. 2010, pp. 1-4.

[65] J. Gomez-Tornero, "Unusual tapering of leaky-wave radiators and their applications," in Proc. 5th Eur. Conf. Antennas Propag. (EUCAP), Rome, Italy, Apr. 2011, pp. 821-824.

[66] A. J. Martinez-Ros, J. L. Gomez-Tornero, and G. Goussetis, "Holographic pattern synthesis with modulated substrate integrated waveguide linesource leaky-wave antennas," IEEE Trans. Antennas Propag., vol. 61, no. 7, pp. 3466-3474, Jul. 2013.

[67] R. Siragusa, E. Perret, P. Lemaitre-Auger, H. Van Nguyen, S. Tedjini, and C. Caloz, "A tapered CRLH interdigital/stub leaky-wave antenna with minimized sidelobe levels," IEEE Antennas Wireless Propag. Lett., vol. 11, pp. 1214-1217, 2012.

[68] C. Caloz, T. Itoh, and A. Rennings, "CRLH metamaterial leaky-wave and resonant antennas," IEEE Antennas Propag. Mag., vol. 50, no. 5, pp. 25-39, Oct. 2008.

[69] L. Liu, C. Caloz, and T. Itoh, "Dominant mode (DM) leaky-wave antenna with backfire-to-endfire scanning capability," Electron. Lett., vol. 38, no. 23 , pp. 1414-1416, Nov. 2002.

[70] J. L. Gómez-Tornero, D. Cañete, and A. Álvarez-Melcón, "Printed-circuit leaky-wave antenna with pointing and illumination flexibility," IEEE Microw. Wireless Compon. Lett., vol. 15, no. 8, pp. 536-538, Aug. 2005.

[71] G. M. Zelinski, G. A. Thiele, M. L. Hastriter, M. J. Havrilla, and A. J. Terzuoli, "Half width leaky wave antennas," IET Microw., Antennas Propag., vol. 1, no. 2, pp. 341-348, Apr. 2007.

[72] J. Xu, W. Hong, H. Tang, Z. Kuai, and K. Wu, "Half-mode substrate integrated waveguide (HMSIW) leaky-wave antenna for millimeter-wave applications," IEEE Antennas Wireless Propag. Lett., vol. 7, pp. 85-88, 2008.

[73] G. Lovat, P. Burghignoli, and D. R. Jackson, "Fundamental properties and optimization of broadside radiation from uniform leaky-wave antennas," IEEE Trans. Antennas Propag., vol. 54, no. 5, pp. 1442-1452, May 2006.

[74] A. J. Martinez-Ros, G. Goussetis, and J. L. Gómez-Tornero, "Pencil beam radiation pattern from a single-layer substrate-integrated waveguide leakywave antenna with simple feeding," IET Microw., Antennas Propag., vol. 9, no. 1, pp. 24-30, Jan. 2015.

[75] S. Majumder, D. R. Jackson, A. A. Oliner, and M. Guglielmi, "The nature of the spectral gap for leaky waves on a periodic strip-grating structure," IEEE Trans. Microw. Theory Techn., vol. 45, no. 12, pp. 2296-2307, Dec. 1997. 
[76] M. Alibakhshikenari, B. S. Virdee, A. Ali, and E. Limiti, "A novel monofilar-archimedean metamaterial inspired leaky-wave antenna for scanning application for passive radar systems," Microw. Opt. Technol. Lett., vol. 60, no. 8, pp. 2055-2060, Aug. 2018.

[77] A. Sanada, M. Kimura, I. Awai, C. Caloz, and T. Itoh, "A planar zeroth-order resonator antenna using a left-handed transmission line," in Proc. 34th Eur. Microw. Conf., Amsterdam, The Netherlands, Oct. 2004, pp. 1341-1344.

[78] M. Alibakhshi-Kenari, M. Naser-Moghadasi, and R. Sadeghzadeh, "The resonating MTM-based miniaturized antennas for wide-band RFmicrowave systems," Microw. Opt. Technol. Lett., vol. 57, no. 10, pp. 2339-2344, Oct. 2015.

[79] J.-Q. Gong and Q.-X. Chu, "Miniaturized microstrip bandpass filter using coupled SCRLH zeroth-order resonators," Microw. Opt. Technol. Lett., vol. 51, no. 12, pp. 2985-2989, Dec. 2009.

[80] A. R. Raslan, A. A. Ibrahim, and A. M. E. Safwat, "Resonant-type antennas loaded with CRLH unit cell," IEEE Antennas Wireless Propag. Lett., vol. 12, pp. 23-26, 2013.

[81] M. Alibakhshi-Kenari, M. Naser-Moghadasi, R. A. Sadeghzadeh, B. S. Virdee, and E. Limiti, "New compact antenna based on simplified CRLH-TL for UWB wireless communication systems," Int. J. RF Microw. Comput.-Aided Eng., vol. 26, no. 3, pp. 217-225, Mar. 2016.

[82] F. Elek and G. V. Eleftheriades, "A two-dimensional uniplanar transmission-line metamaterial with a negative index of refraction," New J. Phys., vol. 7, no. 1, p. 163, 2005.

[83] P. Alitalo, S. Maslovski, and S. Tretyakov, "Three-dimensional isotropic perfect lens based on LC-loaded transmission lines," J. Appl. Phys., vol. 99, no. 6, Mar. 2006, Art. no. 064912.

[84] P. Alitalo, S. Maslovski, and S. Tretyakov, "Experimental verification of the key properties of a three-dimensional isotropic transmission-line superlens," J. Appl. Phys., vol. 99, no. 12, Jun. 2006, Art. no. 124910.

[85] M. Zedler, C. Caloz, and P. Russer, "A 3-D isotropic left-handed metamaterial based on the rotated transmission-line matrix (TLM) scheme," IEEE Trans. Microw. Theory Techn., vol. 55, no. 12, pp. 2930-2941, Dec. 2007.

[86] A. K. Iyer and G. V. Eleftheriades, "A three-dimensional isotropic transmission-line metamaterial topology for free-space excitation," Appl. Phys. Lett., vol. 92, no. 26, Jun. 2008, Art. no. 261106.

[87] G. Kron, "Equivalent Circuit of the Field Equations of Maxwell-I," Proc. IRE, vol. 32, no. 5, pp. 289-299, May 1944.

[88] R. Marques, L. Jelinek, M. J. Freire, J. D. Baena, and M. Lapine, "Bulk metamaterials made of resonant rings," Proc. IEEE, vol. 99, no. 10, pp. $1660-1668$, Oct. 2011

[89] D. O. Guney, T. Koschny, and C. M. Soukoulis, "Intra-connected threedimensionally isotropic bulk negative index photonic metamaterial," Opt. Express, vol. 18, no. 12, pp. 12348-12353, 2010.

[90] C. García-Meca, J. Hurtado, J. Martí, A. Martínez, W. Dickson, an A. V. Zayats, "Low-loss multilayered metamaterial exhibiting a negative index of refraction at visible wavelengths," Phys. Rev. Lett., vol. 106, no. 6, Feb. 2011, Art. no. 067402

[91] C. M. Soukoulis and M. Wegener, "Past achievements and future challenges in the development of three-dimensional photonic metamaterials," Nature Photon., vol. 5, no. 9, pp. 523-530, Sep. 2011.

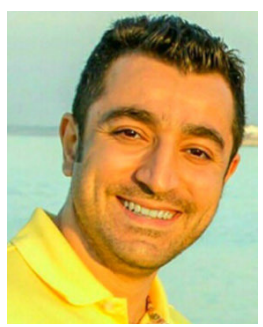

MOHAMMAD ALIBAKHSHIKENARI (Member, IEEE) was born in Iran, in 1988. He received the $\mathrm{Ph} . \mathrm{D}$. degree (Hons.) in electronic engineering from the University of Rome Tor Vergata, Italy, in February 2020.

In 2018 for eight months, he was working with the Antenna System Division, Department of Electrical Engineering, Chalmers University of Technology, Gothenburg, Sweden, as a Ph.D. Visiting Researcher. As well as, his training during the Ph.D. included a research stage in the Swedish company Gap Waves AB that is developing components in a technology. He is currently working as a Postdoctoral Research Fellow with the University of Rome Tor Vergata. During his Ph.D. research period, he has participated in 14 international IEEE conferences over the world, where he has presented 20 articles mostly in oral presentations. During his Ph.D. degree, he was winner of 13 grants for participating in the European Doctoral Schools on Antennas and Metamaterials organized by several European Universities and European School of Antennas (ESoA). He acts as a referee in several high reputed journals and IEEE international conferences. His research interests include antennas and wave-propagations, phased antenna arrays, metamaterials and metasurfaces, synthetic aperture radars (SAR), multiple-input multiple-output (MIMO) systems, waveguide slotted antenna arrays, substrate integrated waveguides (SIWs), impedance matching networks, on-chip antennas, microwave components, millimeter-waves and terahertz integrated circuits, and electromagnetic systems. The above research lines have produced more than 80 publications on refereed-international journals, presentations within international-conferences, and book chapters with a total number of the citations more than $1000, \mathrm{H}$-index of 20 , and I10-indext of 36 reported by the Google Scholar Citation. He was winner of an annual research grant started on November 2019, and to be finalized in November 2020, which has been funded by the Department of Electronic Engineering, University of Rome Tor Vergata.

Dr. Alibakhshikenari was two recipients of the 47th and 48th European Microwave Conference (EuMC) Young Engineer Prize, in 2017, Nuremberg, Germany, and in 2018, Madrid, Spain, where he has presented his articles. He gave an Invited Lecture entitled Metamaterial Applications to Antenna Systems at the Department of Information and Telecommunication Engineering, Incheon National University, Incheon, South Korea, which was in conjunction with the 8th Asia-Pacific Conference on Antennas and Propagation (APCAP 2019), where he was the Chair of the Metamaterial session as well. He is serving as a Guest Editor for a special issue entitled Millimeter-wave and Terahertz Applications of Metamaterials in Applied Sciences. In April 2020, his article entitled High-Gain Metasurface in Polyimide On-Chip Antenna Based on CRLH-TL for Sub Terahertz Integrated Circuits published in Scientific Reports was awarded as best month paper at the University of Bradford, U.K.

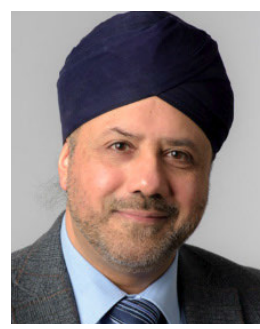

BAL S. VIRDEE (Senior Member, IEEE) received the B.Sc. and M.Phil. degrees in communications engineering from the University of Leeds, U.K., and the Ph.D. degree in electronic engineering from the University of London, U.K. He has worked in industry for various companies, including Philips, U.K., as a Research and Development Engineer and Filtronic Components Ltd. as a Future Products Developer in the area of $\mathrm{RF} /$ microwave communications. He has taught at several academic institutions before joining London Metropolitan University, where he is currently a Professor of microwave-communications with the Faculty of Life Sciences \& Computing, where he is also the Head of the Center for Communications Technology and the Director of London Metropolitan-Microwaves. His research, in collaboration with industry and academia, is in the area of microwave wireless communications encompassing mobile-phones to satellite-technology. He has chaired technical sessions at IEEE international conferences and published numerous research articles. He is an Executive Member of IET's Technical and Professional Network Committee on RF/Microwave-Technology. He is a Fellow of IET.

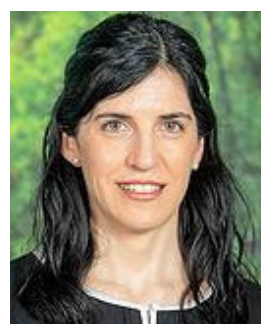

LEYRE AZPILICUETA (Senior Member, IEEE) received the degree in telecommunications engineering, the master's degree in communications, and the Ph.D. degree in telecommunication technologies from the Public University of Navarre (UPNa), Spain, in 2009, 2011, and 2015, respectively. In 2010, she was with the Department of Research and Development, RFID Osés, as a Radio Engineer. She is currently an Associate Professor and a Researcher with the Tecnologico de Monterrey, Monterrey, Mexico. She has over 150 contributions in relevant journals and conference publications. Her research interests include radio propagation, mobile radio systems, ray tracing, and channel modeling. She was a recipient of the IEEE Antennas and Propagation Society Doctoral Research Award, in 2014, the Young Professors and Researchers Santander Universities 2014 Mobility Award, the ECSA 2014 Best Paper Award, the IISA 2015 Best Paper Award, the ISSI 2019 Best Paper Award, the Best Ph.D. from the Colegio Oficial de Ingenieros de Telecomunicación, in 2016, and the N2Women Rising Stars in Computer Networking and Communications 2018 Award. 


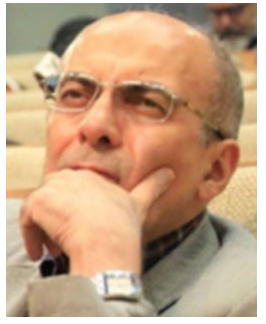

MOHAMMAD NASER-MOGHADASI (Senior Member, IEEE) was born in Saveh, Iran, in 1959. $\mathrm{He}$ received the B.Sc. degree in communication engineering from Leeds Metropolitan University (formerly Leeds Polytechnic), U.K., in 1985, and the Ph.D. degree from the University of Bradford, U.K., in 1993. From 1985 to 1987, he worked as an RF Design Engineer with Gigatech Company, Newcastle Upon Tyne, U.K. From 1987 to 1989, he was awarded a Full Scholarship by the Leeds educational authority to pursue an M.Phil. studying in CAD of microwave circuits. In 1995, he joined the Science and Research Branch, Islamic Azad University, Iran, where he is currently a Full Professor and the Head of the Department of Computer and Electrical Engineering. He has so far published over 200 articles in different journals and conferences. His research interests include microstrip antenna, microwave passive and active circuits, and RF MEMS.

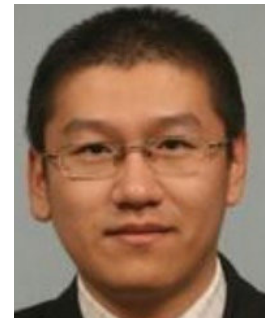

BO LIU (Senior Member, IEEE) received the B.Eng. degree from Tsinghua University, China, in 2008, and the Ph.D. degree from the University of Leuven (KU Leuven), Belgium, in 2012. From 2012 to 2013, he was a Humboldt Research Fellow and was working with the Technical University of Dortmund, Germany. In 2013, he was appointed a Lecturer (Assistant Professor) at Wrexham Glyndwr University, U.K., where he was promoted to a Reader (Associate Professor), in 2016. Since 2020, he has been a Senior Lecturer (Associate Professor) with the University of Glasgow, U.K. He has authored or coauthored one book and more than 60 articles in renowned international journals, edited books, and conference proceedings. His research interests include AI-driven design methodologies of analog/RF integrated circuits, microwave devices, MEMS, evolutionary computation, and machine learning.

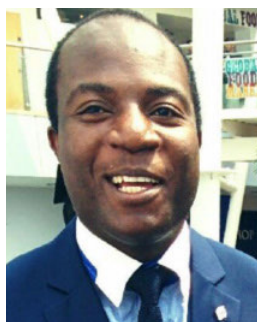

MOBAYODE OLUSOLA AKINSOLU (Member, IEEE) received the M.Sc. degree (Hons.) in electrical and electronic engineering from the University of Bradford, U.K., in 2014, after his undergraduate studies and compulsory national service in Nigeria. He then worked as a Research Fellow (industrial attaché) and a Visiting Researcher at the National Space Research and Development Agency, Nigeria, and the RFID Research Centre, African University of Science and Technology, Nigeria, respectively, until 2016. From 2016 to 2019, he was a Ph.D. scholarship awardee in recognition of a joint project between Wrexham Glyndŵr University, U.K., and the University of Birmingham, U.K. He is currently a Lecturer of electronic and communication engineering with Wrexham Glyndŵr University. His Ph.D. research focused on electromagnetic design automation using surrogate model-assisted evolutionary algorithms. He is a member of the IET and a Registered Electrical Engineer with COREN.

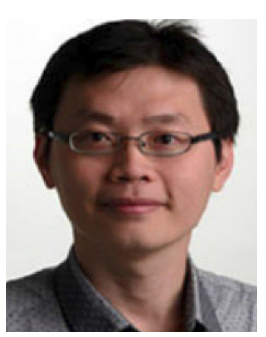

CHAN HWANG SEE (Senior Member, IEEE) received the B.Eng. degree (Hons.) in electronic, telecommunication, and computer engineering and the Ph.D. degree in computational bioelectromagnetics from the University of Bradford, Bradford, U.K., in 2002 and 2007, respectively.

$\mathrm{He}$ is currently a Senior Lecturer (Programme Leader) of electrical and electronic engineering with the School of Engineering, University of Bolton, Bolton, U.K. He is also a Visiting Research Fellow with the School of Engineering and Informatics, University of Bradford. Previously, he was a Senior Research Fellow with the Antennas and Applied Electromagnetics Research Group, University of Bradford. $\mathrm{He}$ has published more than 160 peer-reviewed journal articles and conference papers. He is a coauthor of one book and five book chapters. His research interests include wireless sensor network system design, computational electromagnetism, antennas, and acoustic sensor design.

Dr. See is a Chartered Engineer in the U.K., and a member of the Institution of Engineering and Technology, U.K. He is also a Fellow of The Higher Education Academy. He received two Young Scientist Awards from the International Union of Radio Science and the Asia-Pacific Radio Science Conference, in 2008 and 2010, respectively. He received a certificate of excellence for his successful Knowledge Transfer Partnership with Yorkshire Water on the design and implementation of a wireless sensor system for sewerage infrastructure monitoring, in 2009. He is an Associate Editor of IEEE ACCESS.

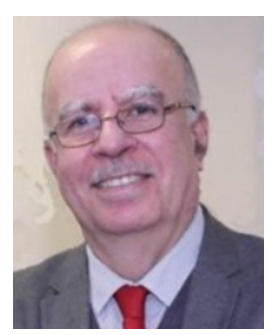

RAED A. ABD-ALHAMEED (Senior Member, IEEE) received the B.Sc. and M.Sc. degrees from Basrah University, Basrah, Iraq, in 1982 and 1985, respectively, and the Ph.D. degree from the University of Bradford, Bradford, U.K., in 1997, all in electrical engineering.

$\mathrm{He}$ is currently a Professor of electromagnetic and radio frequency engineering with the University of Bradford. He is also the Leader of Radio Frequency, Propagation, Sensor Design, and Signal Processing; in addition to leading the Communications research group for years within the School of Engineering and Informatics, University of Bradford. He has long years' research experience in the areas of radio frequency, signal processing, propagations, antennas, and electromagnetic computational techniques. He has published more than 500 academic journal and conference papers; in addition, he is a coauthor of three books and several book chapters. He is also a Principal Investigator for several funded applications to EPSRCs and a Leader of several successful Knowledge Transfer Programmes (KTPs), such as with Arris (previously known as Pace plc), Yorkshire Water plc, Harvard Engineering plc, IETG Ltd., Seven Technologies Group, Emkay Ltd., and Two World Ltd. He has also been a Co-Investigator in several funded research projects, including: H2020 MARIE Sklodowska-CURIE ACTIONS: Innovative Training Networks Secure Network Coding for Next Generation Mobile Small Cells 5G-US, nonlinear and demodulation mechanisms in biological tissue (Department of Health, Mobile Telecommunications \& Health Research Programme, and Assessment of the Potential Direct Effects of Cellular Phones on the Nervous System (EU: collaboration with six other major research organizations across Europe). He has been a Research Visitor with Glyndwr University, Wrexham, U.K., since September 2009, covering the wireless and communications research areas. His research interests include computational methods and optimizations, wireless and mobile communications, sensor design, EMC, beam steering antennas, energy-efficient PAs, and RF predistorter design applications. Dr. Abd-Alhameed is a Fellow of the Institution of Engineering and Technology, U.K., and the Higher Education Academy, and a Chartered Engineer in the U.K. He received the Business Innovation Award for his successful KTP with Pace and Datong companies on the design and implementation of MIMO sensor systems and antenna array design for service localizations. He is the Chair of several successful workshops on Energy Efficient and Reconfigurable Transceivers: Approach Towards Energy Conservation and $\mathrm{CO} 2$ Reduction that addresses the biggest challenges for future wireless systems. He was also appointed as a Guest Editor of IET Science, Measurements and Technology, in 2009 and 2012. 


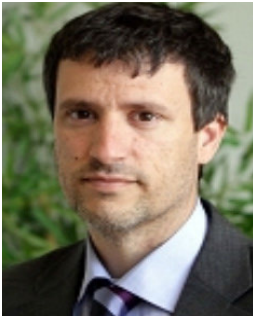

FRANCISCO FALCONE (Senior Member, IEEE) received the degree in telecommunication engineering and the $\mathrm{Ph} . \mathrm{D}$. degree in communication engineering from the Universidad Pública de Navarra (UPNA), Spain, in 1999 and 2005, respectively. From February 1999 to April 2000, he was the Microwave Commissioning Engineer with Siemens-Italtel, deploying microwave access systems. From May 2000 to December 2008, he was a Radio Access Engineer with Telefónica Móviles, performing radio network planning and optimization tasks in mobile network deployment. In January 2009, as a co-founding member, he has been the Director of Tafco Metawireless, a spin-off company from UPNA, until May 2009. In parallel, he was an Assistant Lecturer with the Electrical and Electronic Engineering Department, UPNA, from February 2003 to May 2009. In June 2009, he becomes an Associate Professor with the EE Department, being the Department Head, from January 2012 to July 2018. From January 2018 to May 2018, he was a Visiting Professor with the Kuwait College of Science and Technology, Kuwait. He is also affiliated with the Institute for Smart Cities (ISC), UPNA, which hosts around 140 researchers. He is currently acting as the Head of the ICT Section. He has over 500 contributions in indexed international journals, book chapters, and conference contributions. His research interests are related to computational electromagnetics applied to the analysis of complex electromagnetic scenarios, with a focus on the analysis, design, and implementation of heterogeneous wireless networks to enable context-aware environments. He has been awarded the CST 2003 and CST 2005 Best Paper Award, the Ph.D. Award from the Colegio Oficial de Ingenieros de Telecomunicación (COIT), in 2006, the Doctoral Award UPNA, in 2010, 1st Juan Gomez Peñalver Research Award from the Royal Academy of Engineering of Spain, in 2010, the XII Talgo Innovation Award, in 2012, the IEEE 2014 Best Paper Award, in 2014, the ECSA-3 Best Paper Award, in 2016, and the ECSA-4 Best Paper Award, in 2017.

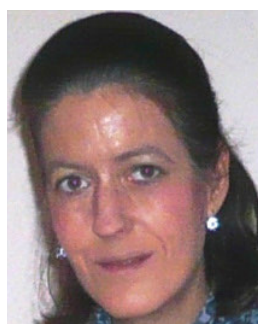

ISABELLE HUYNEN (Senior Member, IEEE) received the $\mathrm{Ph}$.D. degree in applied sciences from the Université catholique de Louvain (UCLouvain), Louvain-la-Neuve, Belgium, in 1994. Since 1999, she has been with FRS-FRNS, Bruxelles, Belgium. She is currently the Research Director and a part-time Professor with UCLouvain. Her current research interests include nanotechnology, nanodevices and nanomaterials for microwave and millimeter wave applications, including metamaterials, antennas, and absorbers.

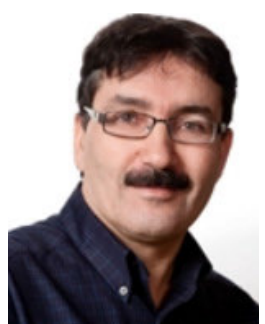

TAYEB A. DENIDNI (Fellow, IEEE) received the M.Sc. and Ph.D. degrees in electrical engineering from Laval University, Quebec City, QC, Canada, in 1990 and 1994, respectively. He was a Professor with the Department of Engineering, Université du Quebec in Rimouski, Rimouski, QC, Canada, from 1994 to 2000, where he founded the Telecommunications Laboratory. Since 2000, he has been with the Institut National de la Recherche Scientifique (INRS), Université du Quebec, Montreal, QC, Canada. He founded the RF Laboratory with INRS-EM, Montreal. He has extensive experience in antenna design and is leading a large research group consisting of three research scientists, eight Ph.D. students, and two M.Sc. students. His current research interests include reconfigurable antennas using EBG and FSS structures, dielectric resonator antennas, metamaterial antennas, adaptive arrays, switched multibeam antenna arrays, ultrawideband antennas, microwave, and development for wireless communications systems. He has served as an Associate Editor for the IEEE Antennas Wireless Propagation Letters, from 2005 to 2007, and the IEEE Transactions on Antennas and Propagation, from 2008 to 2010. Since 2015, he has been serving as an Associate Editor for IET Electronics Letters.

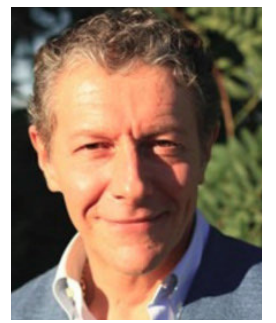

ERNESTO LIMITI (Senior Member, IEEE) has been a Research Assistant and a Teaching Assistant, since 1991, an Associate Professor, since 1998, with the University of Roma Tor Vergata, and a Full Professor of electronics with the Faculty of Engineering, University of Roma Tor Vergata, since 2002. He represents University of Roma Tor Vergata in the governing body of the Microwave Engineering Center for Space Applications (MECSA), an Inter-Universitary Center among several Italian Universities. He has been elected to represent the Industrial Engineering sector in the Academic Senate of the University for the period 2007-2010 and 2010-2013. He is actually the president of the Consortium Advanced Research and Engineering for Space (ARES) formed between the University and two companies. Further, he is actually the President of the Laurea and Laurea Magistrale degrees in Electronic Engineering of the University of Roma Tor Vergata. His research interests include three main lines, all of them belonging to the microwave and millimetre-wave electronics research area. The first one is related to characterisation and modeling for active and passive microwave and millimetre-wave devices. Regarding active devices, the research line is oriented to the smallsignal, and noise and large signal modeling. Regarding passive devices, equivalent-circuit models have been developed for interacting discontinuities in microstrip, for typical MMIC passive components (MIM capacitors) and to waveguide/coplanar waveguide transitions analysis and design. For active devices, new methodologies have been developed for the noise characterisation and the subsequent modeling, and equivalent-circuit modelling strategies have been implemented both for small and large-signal operating regimes for $\mathrm{GaAs}, \mathrm{GaN}, \mathrm{SiC}, \mathrm{Si}$, and InP MESFET/HEMT devices. The second line is related to design methodologies and characterisation methods for low noise circuits. The main focus is on cryogenic amplifiers and devices. Collaborations are currently ongoing with the major radioastronomy institutes all around Europe within the frame of FP6 and FP7 programmes (RadioNet). Finally, the third line is in the analysis methods for nonlinear microwave circuits. In this line, novel analysis methods (Spectral Balance) are developed, together with the stability analysis of the solutions making use of traditional (harmonic balance) approaches. The above research lines have produced more than 250 publications on refereed international journals and presentations within international conferences. He acts as a referee of international journals of the microwave and millimetre wave electronics sector and is in the steering committee of international conferences and workshops. He is actively involved in research activities with many research groups, both European and Italian, and he is in tight collaborations with high-tech italian (Selex - SI, Thales Alenia Space, Rheinmetall, Elettronica S.p.A., Space Engineering, and so on) and foreign (OMMIC, Siemens, UMS, and so on) companies. He contributed, as a researcher and/or as unit responsible, to several National (PRIN MIUR, Madess CNR, and Agenzia Spaziale Italiana) and international (ESPRIT COSMIC, Manpower, Edge, Special Action MEPI, ESA, EUROPA, Korrigan, RadioNet FP6 and FP7, and so on) projects. Regarding teaching activities, he teaches, over his istitutional duties in the frame of the Corso di Laurea Magistrale in Ingegneria Elettronica, Elettronica per lo Spazio within the Master Course in Sistemi Avanzati di Comunicazione e Navigazione Satellitare. He is a member of the committee of the Ph.D. program in telecommunications and microelectronics with the University of Roma Tor Vergata, tutoring an average of four $\mathrm{Ph} . \mathrm{D}$. candidates per year. 